\title{
Manin obstruction to strong approximation for homogeneous spaces
}

\author{
Mikhail Borovoi* and Cyril Demarche
}

\begin{abstract}
For a homogeneous space $X$ (not necessarily principal) of a connected algebraic group $G$ (not necessarily linear) over a number field $k$, we prove a theorem of strong approximation for the adelic points of $X$ in the Brauer-Manin set. Namely, for an adelic point $x$ of $X$ orthogonal to a certain subgroup (which may contain transcendental elements) of the Brauer group $\operatorname{Br}(X)$ of $X$ with respect to the Manin pairing, we prove a strong approximation property for $x$ away from a finite set $S$ of places of $k$. Our result extends a result of Harari for torsors of semiabelian varieties and a result of Colliot-Thélène and Xu for homogeneous spaces of simply connected semisimple groups, and our proof uses those results.
\end{abstract}

Mathematics Subject Classification (2010). Primary 14M17; Secondary 11G35, 14F22, 20G30.

Keywords. Manin obstruction, strong approximation, Brauer group, homogeneous spaces, connected algebraic groups.

\section{Introduction}

Let $k$ be a number field. We denote by $\Omega$ the set of all places of $k$, by $\Omega_{\infty}$ the set of all infinite (archimedean) places of $k$, by $\Omega_{r}$ the set of all real places of $k$, and by $\Omega_{f}$ the set of all finite (nonarchimedean) places of $k$. For a finite set $S \subset \Omega$, we set $k_{S}:=\prod_{v \in S} k_{v}$, where $k_{v}$ denotes the completion of $k$ at $v$. We write $k_{\infty}$ for $k_{\Omega_{\infty}}$. We denote by $\mathbf{A}$ the ring of adèles of $k$ and by $\mathbf{A}^{S}$ the ring of adèles without $S$-components. We have $\mathbf{A}=\mathbf{A}^{S} \times k_{S}$. If $S=\Omega_{\infty}$, we denote by $\mathbf{A}^{f}:=\mathbf{A}^{\Omega_{\infty}}$ the ring of finite adèles. If $X$ is a $k$-variety, we have $X\left(k_{S}\right)=\prod_{v \in S} X\left(k_{v}\right)$ and $X(\mathbf{A})=X\left(\mathbf{A}^{S}\right) \times X\left(k_{S}\right)$. In particular $X(\mathbf{A})=X\left(\mathbf{A}^{f}\right) \times X\left(k_{\infty}\right)$.

Let $X$ be a smooth geometrically integral $k$-variety over a field $k$ of characteristic 0 . Let $\operatorname{Br}(X):=H_{\text {ét }}^{2}\left(X, \mathbb{G}_{m}\right)$ denote the cohomological Brauer group of $X$. We set $\operatorname{Br}_{1}(X):=\operatorname{ker}\left[\operatorname{Br}(X) \rightarrow \operatorname{Br}\left(X \times_{k} \bar{k}\right)\right]$, where $\bar{k}$ is an algebraic closure of $k$.

\footnotetext{
* The first-named author was partially supported by the Israel Science Foundation (grant No. 807/07) and by the Hermann Minkowski Center for Geometry.
} 
Recall that when $k$ is a number field, there exists a canonical pairing (the Manin pairing)

$$
\operatorname{Br}(X) \times X(\mathbf{A}) \rightarrow \mathbb{Q} / \mathbb{Z}, \quad b, x \mapsto\langle b, x\rangle,
$$

see [10], Section 3.1, or [40], Section 5.2. This pairing is additive in $b \in \operatorname{Br}(X)$ and continuous in $x \in X(\mathbf{A})$. If $x \in X(k) \subset X(\mathbf{A})$ or $b$ comes from $\operatorname{Br}(k)$, then $\langle b, x\rangle=0$.

For a subgroup $B \subset \operatorname{Br}(X)$ we denote by $X(\mathbf{A})^{B}$ the set of points of $x \in X(\mathbf{A})$ orthogonal to $B$ with respect to the Manin pairing. We have

$$
X(k) \subset X(\mathbf{A})^{\operatorname{Br}(X)} \subset X(\mathbf{A})^{B} .
$$

One can ask whether any point $x=\left(x_{v}\right) \in X(\mathbf{A})$ which is orthogonal to $\operatorname{Br}(X)$ can be approximated in a certain sense by $k$-rational points.

In this paper we consider the case when $X$ is a homogeneous space of a connected algebraic $k$-group $G$ (not necessarily linear) with connected geometric stabilizers. For such an $X$ and $x=\left(x_{v}\right) \in X(\mathbf{A})^{\mathrm{Br}_{1}(X)}$ it was proved in [7], Appendix, Theorem A.1, that our $X$ has a $k$-point and that $x$ can be approximated by $k$-points in the sense of weak approximation. We used a result of Harari [21] on the Manin obstruction to weak approximation for principal homogeneous spaces of semiabelian varieties. Here, using a result of recent Harari's paper [22] on the Manin obstruction to strong approximation for principal homogeneous spaces of semiabelian varieties together with a recent result of Colliot-Thélène and $\mathrm{Xu}$ [11] on strong approximation for homogeneous spaces of simply connected groups, we prove a theorem on strong approximation for our $x$.

For a connected $k$-group $G$ we write $G^{\text {abvar }}$ for the biggest quotient of $G$ which is an abelian variety, and we write $G^{\text {sc }}$ for the "simply connected semisimple part of $G$ ", see 1.1 below for details.

Theorem 0.1. Let $G$ be a connected algebraic group (not necessarily linear) over a number field $k$. Let $X:=H \backslash G$ be a right homogeneous space of $G$, where $H$ is a connected $k$-subgroup of $G$. Assume that the Tate-Shafarevich group of the maximal abelian variety quotient $G^{\mathrm{abvar}}$ of $G$ is finite. Let $S \supset \Omega_{\infty}$ be a finite set of places of $k$ containing all archimedean places. We assume that $G^{\mathrm{sc}}(k)$ is dense in $G^{\mathrm{sc}}\left(\mathbf{A}^{S}\right)$. Let $x=\left(x_{v}\right) \in X(\mathbf{A})$ be a point orthogonal to $\operatorname{Br}(X)$ with respect to the Manin pairing. Then for any open neighbourhood $U^{S}$ of the projection $x^{S}$ of $x$ to $X\left(\mathbf{A}^{S}\right)$ there exists a rational point $x_{0} \in X(k)$ whose diagonal image in $X\left(\mathbf{A}^{S}\right)$ lies in $u^{S}$. Moreover, we can ensure that for each archimedean place $v$, the points $x_{0}$ and $x_{v}$ lie in the same connected component of $X\left(k_{v}\right)$.

Recall that $G^{\mathrm{sc}}(k)$ is dense in $G^{\mathrm{sc}}\left(\mathbf{A}^{S}\right)$ if and only if for every $k$-simple factor $G_{i}^{\mathrm{sc}}$ of $G^{\mathrm{sc}}$ the group $G_{i}^{\mathrm{sc}}\left(k_{S}\right)$ is noncompact (a theorem of Kneser and Platonov, cf. [36], Theorem 7.12). 
Theorem 0.1 extends a result of Harari ([22], Theorem 4) and a result of ColliotThélène and Xu ([11], Theorem 3.7 (b)).

In Theorem 0.1 we assume that our adelic point $x$ is orthogonal to the whole Brauer group $\operatorname{Br}(X)$. Actually it is sufficient to require that $x$ were orthogonal to a certain subgroup $\operatorname{Br}_{1}(X, G) \subset \operatorname{Br}(X)$. In general this subgroup $\operatorname{Br}_{1}(X, G)$ contains transcendental elements (i.e. is not contained in $\left.\operatorname{Br}_{1}(X)\right)$. Note that Theorem 0.1 with $\operatorname{Br}_{1}(X)$ instead of $\operatorname{Br}(X)$ would be false, see Counter-example 1.6 below. However this theorem still holds with $\operatorname{Br}_{1}(X)$ instead of $\operatorname{Br}(X)$, if $S$ contains at least one nonarchimedean place:

Theorem 0.2. Let $G$ be a connected algebraic group (not necessarily linear) over a number field $k$. Let $X:=H \backslash G$ be a right homogeneous space of $G$, where $H$ is a connected $k$-subgroup of $G$. Assume that the Tate-Shafarevich group of the maximal abelian variety quotient $G^{\text {abvar }}$ of $G$ is finite. Let $S \supset \Omega_{\infty}$ be a finite set of places of $k$ containing all archimedean places and at least one nonarchimedean place. We assume that $G^{\mathrm{sc}}(k)$ is dense in $G^{\mathrm{sc}}\left(\mathbf{A}^{S}\right)$. Let $x=\left(x_{v}\right) \in X(\mathbf{A})$ be a point orthogonal to $\mathrm{Br}_{1}(X)$ with respect to the Manin pairing. Then for any open neighbourhood $U^{S}$ of the projection $x^{S}$ of $x$ to $X\left(\mathbf{A}^{S}\right)$ there exists a rational point $x_{0} \in X(k)$ whose diagonal image in $X\left(\mathbf{A}^{S}\right)$ lies in $U^{S}$. Moreover, we can ensure that for each archimedean place $v$, the points $x_{0}$ and $x_{v}$ lie in the same connected component of $X\left(k_{v}\right)$.

Remark 0.3. Let $X$ be a right homogeneous space of a connected group $k$-group $G$ over a number field $k$ such that the stabilizers of the geometric points are connected. By [7], Theorem A.1, if there exists $x \in X(\mathbf{A})$ which is orthogonal to $\operatorname{Br}_{1}(X)$, then the variety $X$ must have a $k$-point, hence $X=H \backslash G$, where $H$ is a connected subgroup of $G$. Therefore we could reformulate Theorems $0.1,0.2,1.4$ and 1.7 for a general homogeneous space $X$ of $G$ (without assuming that $X$ is of the form $X=H \backslash G$ ).

Our proof is somewhat similar to that of Theorem A.1 of [7]. We use the reductions and constructions of Subsections 3.1 and 3.3 of [7] in order to reduce the assertion to the case when $X$ is a $k$-torsor under a semiabelian variety (treated by Harari [22]) and to the case when $X$ is a homogeneous space of a simply connected semisimple group with connected geometric stabilizers (the Hasse principle was proved in [4]; for strong approximation, see Theorem 6.1 below, which is actually due to Colliot-Thélène and $\mathrm{Xu}[11])$. 


\section{Main results}

1.1. Let $G$ be a connected algebraic group (not necessarily linear) over a field $k$ of characteristic 0 . Then $G$ fits into a canonical short exact sequence

$$
1 \rightarrow G^{\text {lin }} \rightarrow G \rightarrow G^{\text {abvar }} \rightarrow 1,
$$

where $G^{\text {lin }}$ is a connected linear $k$-group and $G^{\text {abvar }}$ is an abelian variety over $k$. We use the following notation:

$G^{\mathrm{u}}$ is the unipotent radical of $G^{\text {lin }}$;

$G^{\text {red }}:=G^{\text {lin }} / G^{\mathrm{u}}$, it is a reductive $k$-group;

$G^{\text {ss }}$ is the commutator subgroup of $G^{\text {red }}$, it is a semisimple $k$-group;

$G^{\text {sc }}$ is the universal covering of $G^{\text {ss }}$, it is a simply connected semisimple $k$-group;

$G^{\text {tor }}:=G^{\text {red }} / G^{\text {ss }}$, it is a $k$-torus;

$G^{\text {ssu }}:=\operatorname{Ker}\left(G^{\text {lin }} \rightarrow G^{\text {tor }}\right)$, it is an extension of $G^{\text {ss }}$ by $G^{\mathrm{u}}$;

$G^{\mathrm{sab}}:=\left(G / G^{\mathrm{u}}\right) / G^{\mathrm{ss}}$, it is a semiabelian variety over $k$, it fits into a short exact sequence

$$
1 \rightarrow G^{\mathrm{tor}} \rightarrow G^{\mathrm{sab}} \rightarrow G^{\mathrm{abvar}} \rightarrow 1 .
$$

We define the group $G^{\text {scu }}$ as the fibre product $G^{\mathrm{scu}}:=G^{\mathrm{sc}} \times{ }_{G^{\mathrm{red}}} G^{\text {lin }}$, it fits into an exact sequence

$$
1 \rightarrow G^{\mathrm{u}} \rightarrow G^{\mathrm{scu}} \rightarrow G^{\mathrm{sc}} \rightarrow 1 .
$$

We have a canonical homomorphism $G^{\text {scu }} \rightarrow G^{\text {lin }} \rightarrow G$.

1.2. Let $X$ be a smooth geometrically integral $k$-variety. We write $\bar{X}$ for $X \times_{k} \bar{k}$, where $\bar{k}$ is a fixed algebraic closure of $k$. Recall that $\operatorname{Br}(X)$ is the cohomological Brauer group of $X$ and that $\operatorname{Br}_{1}(X)=\operatorname{ker}[\operatorname{Br}(X) \rightarrow \operatorname{Br}(\bar{X})]$. We set $\operatorname{Br}_{\mathrm{a}}(X):=$ coker $\left[\operatorname{Br}(k) \rightarrow \mathrm{Br}_{1}(X)\right]$. If $x_{0} \in X(k)$ is a $k$-point of $X$, we set $\mathrm{Br}_{x_{0}}(X):=$ $\operatorname{ker}\left[x_{0}^{*}: \operatorname{Br}(X) \rightarrow \operatorname{Br}(k)\right]$ and $\operatorname{Br}_{1, x_{0}}(X):=\operatorname{ker}\left[x_{0}^{*}: \operatorname{Br}_{1}(X) \rightarrow \operatorname{Br}(k)\right]$. We have a canonical isomorphism $\mathrm{Br}_{1, x_{0}}(X) \stackrel{\sim}{\longrightarrow} \operatorname{Br}_{\mathrm{a}}(X)$.

Let $X=H \backslash G$ be a homogeneous space of a connected $k$-group $G$. Let $x_{0} \in$ $X(k)$. Consider the map $\pi_{x_{0}}: G \rightarrow X, g \mapsto x_{0} . g$, it induces a homomorphism $\pi_{x_{0}}^{*}: \operatorname{Br}(X) \rightarrow \operatorname{Br}(G)$. Consider the commutative diagram

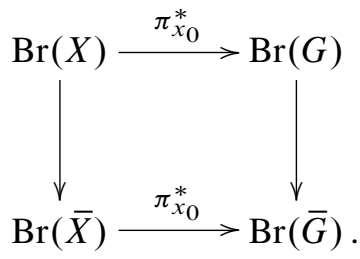

Let $\operatorname{Br}_{1}(X, G)$ denote the kernel of any of the two equal composed homomorphisms $\operatorname{Br}(X) \rightarrow \operatorname{Br}(\bar{G})$. In other words, $\operatorname{Br}_{1}(X, G)$ is the subgroup of elements $b \in \operatorname{Br}(X)$ 
such that $\pi_{x_{0}}^{*}(b) \in \operatorname{Br}_{1}(G)$. We show in Lemma 4.4 below, that $\operatorname{Br}_{1}(X, G)$ does not depend on $x_{0}$. Let

$$
\begin{aligned}
\operatorname{Br}_{1, x_{0}}(X, G): & =\operatorname{ker}\left[x_{0}^{*}: \operatorname{Br}_{1}(X, G) \rightarrow \operatorname{Br}(k)\right] \\
& =\left\{b \in \operatorname{Br}(X) \mid \pi_{x_{0}}^{*}(b) \in \operatorname{Br}_{1, e}(G)\right\} .
\end{aligned}
$$

It is easy to see that the structure map $X \rightarrow \operatorname{Spec}(k)$ induces an embedding $\operatorname{Br}(k) \hookrightarrow$ $\operatorname{Br}_{1}(X, G)$ (because $X$ has a $k$-point) and that $\operatorname{Br}_{1}(X, G)=\operatorname{Br}_{1, x_{0}}(X, G)+\operatorname{Br}(k)$. It follows that an adelic point $x \in X(\mathbf{A})$ is orthogonal to $\operatorname{Br}_{1}(X, G)$ with respect to the Manin pairing if and only if it is orthogonal to $\operatorname{Br}_{1, x_{0}}(X, G)$.

1.3. Let $X$ be a smooth geometrically integral $k$-variety over a number field $k$. We denote by $X(\mathbf{A})$. the set $X\left(\mathbf{A}^{f}\right) \times \prod_{v \in \Omega_{\infty}} \pi_{0}\left(X\left(k_{v}\right)\right)$, where $\pi_{0}\left(X\left(k_{v}\right)\right)$ is the set of connected components of $X\left(k_{v}\right)$. The set $X(\mathbf{A}) \bullet$ has a natural topology, which we call the adelic topology. We have a canonical continuous map $X(\mathbf{A}) \rightarrow X(\mathbf{A}) \bullet$ and a canonical embedding $X(k) \hookrightarrow X(\mathbf{A})$ •. The pairing (1) of the Introduction induces a pairing

$$
\operatorname{Br}(X) \times X(\mathbf{A}) \bullet \rightarrow \mathbb{Q} / \mathbb{Z}
$$

For a subgroup $B \subset \operatorname{Br}(X)$ we denote by $\left(X(\mathbf{A})_{\bullet}\right)^{B}$ the set of points of $x \in X(\mathbf{A})$. orthogonal to $B$ with respect to the Manin pairing.

Let $X$ be a homogeneous space of a connected $k$-group $G$. Then $G(\mathbf{A})$ acts on $X(\mathbf{A})$ and on $X(\mathbf{A})$.

Main Theorem 1.4. Let $G$ be a connected algebraic group (not necessarily linear) over a number field $k$. Let $X:=H \backslash G$ be a right homogeneous space of $G$, where $H$ is a connected $k$-subgroup of $G$. Assume that the Tate-Shafarevich group of the maximal abelian variety quotient $G^{\text {abvar }}$ of $G$ is finite. Let $S \supset \Omega_{\infty}$ be a finite set of places of $k$ containing all archimedean places. We assume that $G^{\mathrm{sc}}(k)$ is dense in $G^{\mathrm{sc}}\left(\mathbf{A}^{S}\right)$. Set $S_{f}:=S \cap \Omega_{f}=S \backslash \Omega_{\infty}$. Then the set $\left(X(\mathbf{A})_{\bullet}\right)^{\mathrm{Br}_{1}(X, G)}$ coincides with the closure of the set $X(k) . G^{\mathrm{scu}}\left(k_{S_{f}}\right)$ in $X(\mathbf{A}) \bullet$ for the adelic topology.

Remark 1.5. If $x \in X(\mathbf{A})$. is not orthogonal to $\operatorname{Br}_{1}(X, G)$, we regard it as an obstruction to strong approximation for $x$ (this is the Manin obstruction to strong approximation from the title of the paper). Indeed, then by the trivial part of Main Theorem 1.4 the point $x$ does not belong to the closure of $X(k) \cdot G^{\mathrm{scu}}\left(k_{S_{f}}\right)$. We interpret the nontrivial part of this theorem as an assertion that under certain assumptions the Manin obstruction is the only obstruction to strong approximation for $x$ and for its projection $x^{S} \in X\left(\mathbf{A}^{S}\right)$. Indeed, if there is no Manin obstruction, i.e. $x$ is orthogonal to $\operatorname{Br}_{1}(X, G)$, then by the nontrivial part of Main Theorem 1.4 the point $x$ belongs to the closure of $X(k) \cdot G^{\text {scu }}\left(k_{S_{f}}\right)$, and its projection $x^{S} \in X\left(\mathbf{A}^{S}\right)$ belongs to the closure of $X(k)$ in $X\left(\mathbf{A}^{S}\right)$. 
1.6. Counter-example with $\operatorname{Br}_{1}(X)$ instead of $\operatorname{Br}(X)$. Above we stated the main theorem about the Manin obstruction related to the subgroup $\operatorname{Br}_{1}(X, G) \subset \operatorname{Br}(X)$. We are interested also in the algebraic Manin obstruction, that is, the obstruction coming from the subgroup $\operatorname{Br}_{1}(X) \subset \operatorname{Br}(X)$. We can easily see that in general the algebraic Manin obstruction is not the only obstruction to strong approximation.

First we notice that in general $\operatorname{Br}_{1}(X) \varsubsetneqq \operatorname{Br}_{1}(X, G)$. Indeed, assume that $G$ is semisimple and simply connected and that $H$ is connected semisimple but not simply connected. In particular, $H$ fits into an exact sequence

$$
1 \rightarrow \mu_{H} \rightarrow H^{\mathrm{sc}} \rightarrow H \rightarrow 1,
$$

where $\mu_{H}$ is finite and abelian. Consider $X:=H \backslash G$. Then we know by [38], Proposition 6.9(iv), that the groups $\operatorname{Pic}(G)$ and $\operatorname{Br}_{1, e}(G)$ are trivial. By [11], Proposition 2.10 (ii), we have a canonical isomorphism $\operatorname{Pic}(H) \cong \mathrm{Br}_{x_{0}}(X)$, where $x_{0} \in X(k)$ is the image of $e \in G(k)$. Since $H$ is semisimple, by the exact sequence in [38], Lemma 6.9(i), we know that the map $\operatorname{Pic}(H) \rightarrow \operatorname{Pic}(\bar{H})$ is injective, therefore any non-trivial element of $\operatorname{Br}_{x_{0}}(X)$ is a transcendental element of $\operatorname{Br}(X)$, i.e. is not killed in $\operatorname{Br}(\bar{X})$. We see that $\operatorname{Br}_{1, x_{0}}(X)=0$, hence $\operatorname{Br}_{\mathrm{a}}(X)=$ 0 . In addition, Proposition 2.6 (iii) in [11] (or the corollary in the introduction of [18]) implies that $\operatorname{Br}(\bar{G})=0$, hence $\mathrm{Br}_{1, x_{0}}(X, G)=\mathrm{Br}_{x_{0}}(X)$. Therefore in this case $\mathrm{Br}_{1}(X) / \operatorname{Br}(k)=\mathrm{Br}_{\mathrm{a}}(X)=0$, while $\mathrm{Br}_{1}(X, G) / \operatorname{Br}(k) \cong \mathrm{Br}_{1, x_{0}}(X, G) \cong$ $\operatorname{Pic}(H) \cong \widehat{\mu_{H}}(k)$. We see that $\operatorname{Br}_{1}(X, G) \not \subset \operatorname{Br}_{1}(X)$ if $\widehat{\mu_{H}}(k) \neq 0$.

An explicit example is given by $H=\mathrm{SO}_{n} \subset \mathrm{SL}_{n}=G$ for $n \geq 3$. In this case $\mu_{H}=\mu_{2}$ and $\widehat{\mu_{H}}(k)=\mathbb{Z} / 2 \mathbb{Z} \neq 0$, hence $\operatorname{Br}_{1}(X, G) \not \subset \operatorname{Br}_{1}(X)$. We take $k$ to be a totally imaginary number field, e.g. $k=\mathbb{Q}(i)$. Take $S=\Omega_{\infty}$, then $\mathbf{A}^{S}=\mathbf{A}^{f}$. We show that in this case the algebraic Manin obstruction is not the only obstruction to strong approximation away from $S$.

We may and shall identify $X:=H \backslash G$ with the variety of symmetric $n \times n$ matrices $T$ with determinant 1 . Then an element of $X(\mathbf{A})$ can be written as $\left(T_{v}\right)_{v \in \Omega}$, where $T_{v}$ is a symmetric $n \times n$-matrix with determinant 1 over $k_{v}$. Let $\varepsilon_{v}\left(T_{v}\right) \in\{ \pm 1\}$ denote the Hasse invariant of the quadratic form defined by $T_{v}$ (see for instance [33], p. 167, before Example 63:14). Note that for $v \in \Omega_{\infty}$ we have $k_{v} \cong \mathbb{C}$, hence $\varepsilon_{v}\left(T_{v}\right)=1$. For $T^{f} \in X\left(\mathbf{A}^{f}\right)$ set

$$
\varepsilon^{f}\left(T^{f}\right)=\prod_{v \in \Omega_{f}} \varepsilon_{v}\left(T_{v}\right),
$$

then $\varepsilon^{f}$ is a continuous function on $X\left(\mathbf{A}^{f}\right)$ with values \pm 1 . For $T_{0} \in X(k)$ we have

$$
\varepsilon^{f}\left(T_{0}\right)=\prod_{v \in \Omega_{f}} \varepsilon_{v}\left(T_{0}\right)=\prod_{v \in \Omega_{f}} \varepsilon_{v}\left(T_{0}\right) \prod_{v \in \Omega_{\infty}} \varepsilon_{v}\left(T_{0}\right)=\prod_{v \in \Omega} \varepsilon_{v}\left(T_{0}\right)=1,
$$

because for all $v \in \Omega_{\infty}$ we have $\varepsilon_{v}\left(T_{0}\right)=1$, and because by [33], Theorem 72:1, the last product equals 1 . Since $\varepsilon^{f}$ is a continuous function, for any $T^{f}=\left(T_{v}\right) \in X\left(\mathbf{A}^{f}\right)$ 
lying in the closure of $X(k)$ we have $\varepsilon^{f}\left(T^{f}\right)=1$. We show below that there exists $U^{f} \in X\left(\mathbf{A}^{f}\right)$ with $\varepsilon^{f}\left(U^{f}\right)=-1$.

Fix $v_{0} \in \Omega_{f}$. Let $U_{v_{0}} \in X\left(k_{v_{0}}\right)$ be a symmetric matrix with determinant 1 with $\varepsilon_{v_{0}}\left(U_{v_{0}}\right)=-1$ (there exists such a symmetric matrix, see [33], Theorem 63:22). For $v \in \Omega_{f} \backslash\left\{v_{0}\right\}$ set $U_{v}:=\operatorname{diag}(1, \ldots, 1) \in X\left(k_{v}\right)$, then $\varepsilon_{v}\left(U_{v}\right)=1$. We obtain an element $U^{f}=\left(U_{v}\right)_{v \in \Omega_{f}} \in X\left(\mathbf{A}^{f}\right)$ with $\varepsilon^{f}\left(U^{f}\right)=-1$. We see that $U^{f}$ does not lie in the closure of $X(k)$ in $X\left(\mathbf{A}^{f}\right)$.

It is well known that for our $G=\mathrm{SL}_{n}$ and $S=\Omega_{\infty}$, the group $G(k)$ is dense in $G\left(\mathbf{A}^{S}\right)=G\left(\mathbf{A}^{f}\right)$. We have $\operatorname{Br}_{1}(X)=\operatorname{Br}(k)$, hence all the points of $X(\mathbf{A}) \bullet=$ $X\left(\mathbf{A}^{f}\right)$ are orthogonal to $\operatorname{Br}_{1}(X)$, in particular our point $U^{f}$. However, $U^{f}$ does not lie in the closure of $X(k)$ in $X\left(\mathbf{A}^{f}\right)=X\left(\mathbf{A}^{S}\right)=X(\mathbf{A})$.

Of course, our $U^{f}$ is not orthogonal to $\operatorname{Br}_{1}(X, G)$ (otherwise it would lie in the closure of $X(k)$ by Main Theorem 1.4). Indeed, consider the map

$$
m: X\left(\mathbf{A}^{f}\right)=X(\mathbf{A}) \bullet \longrightarrow \operatorname{Hom}\left(\operatorname{Br}_{1}(X, G) / \operatorname{Br}(k), \mathbb{Q} / \mathbb{Z}\right)=\mathbb{Z} / 2 \mathbb{Z}
$$

induced by the Manin pairing (2) from 1.3. It can be shown that this map coincides with the $\operatorname{map} \varepsilon^{f}: X\left(\mathbf{A}^{f}\right) \rightarrow\{ \pm 1\}$ under the canonical identification $\mathbb{Z} / 2 \mathbb{Z} \cong\{ \pm 1\}$. It follows that $m\left(U^{f}\right)=1+2 \mathbb{Z} \in \mathbb{Z} / 2 \mathbb{Z}$, hence $U^{f}$ is not orthogonal to $\operatorname{Br}_{1}(X, G)$.

The above counter-example shows that Main Theorem 1.4 does not hold with $\operatorname{Br}_{1}(X)$ instead of $\operatorname{Br}_{1}(X, G)$. Nevertheless, we can prove a similar result about the algebraic Manin obstruction, assuming that $S$ contains at least one nonarchimedean place.

Theorem 1.7. Let $G$ be a connected algebraic group (not necessarily linear) over a number field $k$. Let $X:=H \backslash G$ be a right homogeneous space of $G$, where $H$ is a connected $k$-subgroup of $G$. Assume that the Tate-Shafarevich group of the maximal abelian variety quotient $G^{\text {abvar }}$ of $G$ is finite. Let $x \in X(\mathbf{A})$ be an adelic point orthogonal to $\mathrm{Br}_{1} X$ with respect to the Manin pairing. Let $S \supset \Omega_{\infty}$ be a finite set of places of $k$ containing all archimedean places and at least one nonarchimedean place $v_{0}$. We assume that $G^{\mathrm{sc}}(k)$ is dense in $G^{\mathrm{sc}}\left(\mathbf{A}^{S}\right)$. Set $S_{f}^{\prime}:=S \backslash\left(\Omega_{\infty} \cup\left\{v_{0}\right\}\right)$. We write $X\left(\mathbf{A}^{\left\{v_{0}\right\}}\right) \bullet$ for $X\left(\mathbf{A}^{\Omega_{\infty} \cup\left\{v_{0}\right\}}\right) \times \prod_{v \in \Omega_{\infty}} \pi_{0}\left(X\left(k_{v}\right)\right)$. Then the projection $x^{\left\{v_{0}\right\}} \in X\left(\mathbf{A}^{\left\{v_{0}\right\}}\right)$. of $x$ lies in the closure of the set $X(k) \cdot G^{\mathrm{scu}}\left(k_{S_{f}^{\prime}}\right)$ in $X\left(\mathbf{A}^{\left\{v_{0}\right\}}\right) \bullet$ for the adelic topology.

Theorem 0.1 follows from Theorem 1.4, and Theorem 0.2 follows from Theorem 1.7. 


\section{Sansuc's exact sequence}

2.1. Let $k$ be a field of characteristic 0 .

Let $\pi: Y \rightarrow X$ be a (left) torsor under a connected linear $k$-group $H$. We define $\operatorname{Br}_{1}(X, Y)$ to be the following group:

$$
\operatorname{Br}_{1}(X, Y):=\left\{b \in \operatorname{Br}(X): \pi^{*}(b) \in \operatorname{Br}_{1}(Y)\right\},
$$

and if $y \in Y(k), x=\pi(y)$, define $\operatorname{Br}_{1, x}(X, Y)$ to be

$$
\operatorname{Br}_{1, x}(X, Y):=\operatorname{ker}\left[x^{*}: \operatorname{Br}_{1}(X, Y) \rightarrow \operatorname{Br}(k)\right]=\left\{b \in \operatorname{Br}(X): \pi^{*}(b) \in \operatorname{Br}_{1, y}(Y)\right\} .
$$

We denote by

$$
\langle,\rangle: \operatorname{Br}(X) \times X(k) \rightarrow \operatorname{Br}(k):(b, x) \mapsto b(x)
$$

the evaluation map.

2.2. Before recalling the result of Sansuc, we give a few more definitions and notations. Let $\mathcal{A}$ be an abelian category and $F: \underline{\mathrm{Var}} / k \rightarrow \mathcal{A}$ be a contravariant functor from the category of $k$-varieties to $\mathcal{A}$. If $X$ and $Y$ are $k$-varieties, the projections $p_{X}, p_{Y}: X \times_{k} Y \rightarrow X, Y$ induce a morphism in $\mathcal{A}$ (see [38], Section 6.b):

$$
F\left(p_{X}\right)+F\left(p_{Y}\right): F(X) \oplus F(Y) \rightarrow F\left(X \times_{k} Y\right)
$$

such that

$$
F\left(p_{X}\right)+F\left(p_{Y}\right)=F\left(p_{X}\right) \circ \pi_{X}+F\left(p_{Y}\right) \circ \pi_{Y},
$$

where $\pi_{X}, \pi_{Y}$ are the projections $F(X) \oplus F(Y) \rightarrow F(X), F(Y)$ and the group law in the right-hand side is the law in $\operatorname{Hom}\left(F(X) \oplus F(Y), F\left(X \times_{k} Y\right)\right)$.

Let $m: X \times_{k} Y \rightarrow Y$ be a morphism of $k$-varieties. Assume that the morphism $F\left(p_{X}\right)+F\left(p_{Y}\right)$ is an isomorphism. We define a map

$$
\varphi: F(Y) \rightarrow F\left(X \times_{k} Y\right) \rightarrow F(X) \oplus F(Y) \rightarrow F(X)
$$

by the formula

$$
\varphi:=\pi_{X} \circ\left(F\left(p_{X}\right)+F\left(p_{Y}\right)\right)^{-1} \circ F(m)
$$

(see [38], (6.4.1)).

Lemma 2.3. Let $F: \underline{\operatorname{Var}} / k \rightarrow \mathcal{A}$ be a contravariant functor. Let $X, Y$ be two $k$-varieties, $m: X \times_{k} Y \rightarrow Y$ be a $k$-morphism. Assume that:

- $F(\operatorname{Spec}(k))=0$.

- $F\left(p_{X}\right)+F\left(p_{Y}\right): F(X) \oplus F(Y) \rightarrow F\left(X \times_{k} Y\right)$ is an isomorphism. 
- There exists $x \in X(k)$ such that the morphism $m(x,):. Y \rightarrow Y$ is the identity of $Y$.

Then $F(m)=F\left(p_{X}\right) \circ \varphi+F\left(p_{Y}\right): F(Y) \rightarrow F\left(X \times_{k} Y\right)$.

Proof. Consider the morphism $x_{Y}: Y \rightarrow X \times_{k} Y$ defined by $x$. Then $F\left(x_{Y}\right) \circ$ $F\left(p_{X}\right)=0$, since the morphism $p_{X} \circ x_{Y}: Y \rightarrow X$ factors through $x: \operatorname{Spec}(k) \rightarrow X$ and $F(\operatorname{Spec}(k))=0$. Since $p_{Y} \circ x_{Y}=\mathrm{id}_{Y}$, we have $F\left(x_{Y}\right) \circ F\left(p_{Y}\right)=\mathrm{id}$, and the third assumption of the lemma implies that $F\left(x_{Y}\right) \circ F(m)=$ id. Therefore, we deduce that

$$
F\left(x_{Y}\right) \circ\left(F\left(p_{X}\right)+F\left(p_{Y}\right)\right)=\pi_{Y}: F(X) \oplus F(Y) \rightarrow F(Y),
$$

hence

$$
\pi_{Y} \circ\left(F\left(p_{X}\right)+F\left(p_{Y}\right)\right)^{-1} \circ F(m)=F\left(x_{Y}\right) \circ F(m)=\operatorname{id}_{F(Y)} .
$$

But by (3) we have

$$
\begin{aligned}
F(m)=F & \left(p_{X}\right) \circ \pi_{X} \circ\left(F\left(p_{X}\right)+F\left(p_{Y}\right)\right)^{-1} \circ F(m) \\
& +F\left(p_{Y}\right) \circ \pi_{Y} \circ\left(F\left(p_{X}\right)+F\left(p_{Y}\right)\right)^{-1} \circ F(m),
\end{aligned}
$$

so (4) and (5) give exactly

$$
F(m)=F\left(p_{X}\right) \circ \varphi+F\left(p_{Y}\right) .
$$

We shall apply those constructions and this lemma to the functors $F=\operatorname{Pic}($.$) and$ $F=\mathrm{Br}_{\mathrm{a}}($.$) and to the morphism m: H \times Y \rightarrow Y$ defined by an action of an algebraic group $H$ on a variety $Y$. In this context, those functors satisfy the assumptions of Lemma 2.3 by [38], Lemma 6.6.

We now recall Sansuc's result.

Proposition 2.4 ([38], Proposition 6.10). Let $k$ be a field of characteristic zero, $H$ a connected linear $k$-group, $X$ a smooth $k$-variety and $\pi: Y \stackrel{H}{\rightarrow} X$ a torsor under $H$. Then we have a functorial exact sequence:

$$
\operatorname{Pic}(Y) \stackrel{\varphi_{1}}{\longrightarrow} \operatorname{Pic}(H) \stackrel{\Delta_{Y / X}^{\prime}}{\longrightarrow} \operatorname{Br}(X) \stackrel{\pi^{*}}{\longrightarrow} \operatorname{Br}(Y) \stackrel{m^{*}-p_{Y}^{*}}{\longrightarrow} \operatorname{Br}(H \times Y) .
$$

Here $m: H \times Y \rightarrow Y$ denotes the left action of $H$ on $Y, p_{Y}: H \times Y \rightarrow Y$ the natural projection and $\Delta_{Y / X}^{\prime}: \operatorname{Pic}(H) \rightarrow \operatorname{Br}(X)$ is a map defined in the proof of Proposition 6.10 in [38]. For the definition of the map $\varphi_{1}$, see (4) or [38], (6.4.1).

Proof. For the part of the exact sequence up to $\operatorname{Br}(X) \stackrel{\pi^{*}}{\longrightarrow} \operatorname{Br}(Y)$ see [38], Proposition 6.10. Concerning the last map $\operatorname{Br}(Y) \rightarrow \operatorname{Br}(H \times Y)$, it implicitly appears in Sansuc's paper in the last term of the exact sequence

$$
0 \rightarrow \check{H}^{1}(Y / X, \mathcal{P i c}) \rightarrow H^{2}\left(X, \mathbb{G}_{m}\right) \stackrel{p}{\rightarrow} \check{H}^{0}\left(Y / X, \mathcal{B r}^{\prime}\right) \rightarrow 0
$$


(the second sequence of the four short exact sequences on page 45). By definition

$$
\check{H}^{0}\left(Y / X, \mathcal{B} r^{\prime}\right)=\operatorname{ker}\left[\operatorname{Br}(Y) \stackrel{\operatorname{pr}_{1}^{*}-\mathrm{pr}_{2}^{*}}{\longrightarrow} \operatorname{Br}\left(Y \times_{X} Y\right)\right],
$$

where $\mathrm{pr}_{i}: Y \times_{X} Y \rightarrow Y$ denote the two projections. Since $Y \rightarrow X$ is a torsor, we have a canonical isomorphism $H \times Y \rightarrow Y \times_{X} Y$ defined by $(h, y) \mapsto(m(h, y), y)$. Sansuc noticed that the maps $\mathrm{pr}_{1}$ and $\mathrm{pr}_{2}$ correspond under this isomorphism to the maps $m$ and $p_{Y}$, respectively (see [38], the formulas for the faces of the simplicial system on page 44 before Lemma 6.12). Thus we see that

$$
\check{H}^{0}\left(Y / X, \mathcal{B} r^{\prime}\right)=\operatorname{ker}\left[\operatorname{Br}(Y) \stackrel{m^{*}-p_{Y}^{*}}{\longrightarrow} \operatorname{Br}(H \times Y)\right] .
$$

A computation using the Čech spectral sequence (6.12.0) in [38] shows that the map $\operatorname{Br}(X) \rightarrow \operatorname{Br}(Y)$ defined by the composition

$$
H^{2}\left(X, \mathbb{G}_{m}\right) \stackrel{p}{\rightarrow} \check{H}^{0}\left(Y / X, \mathcal{B} r^{\prime}\right) \subset H^{2}\left(Y, \mathbb{G}_{m}\right)
$$

is the pullback morphism $\pi^{*}: \operatorname{Br}(X) \rightarrow \operatorname{Br}(Y)$. This concludes the proof of the exactness of (6).

The exact sequence in the above proposition will be very useful in the following, but we need another one: we need a version of this exact sequence with the map $\Delta_{Y / X}: \operatorname{Pic}(H) \rightarrow \operatorname{Br}(X)$, defined in [11] before Proposition 2.3. We recall here the definition of $\Delta_{Y / X}$ due to Colliot-Thélène and $\mathrm{Xu}$.

2.5. Definition of $\boldsymbol{\Delta}_{\boldsymbol{Y} / \boldsymbol{X}}$. We use the above notation. Since $H$ is connected, we have a canonical isomorphism $c_{H}: \operatorname{Ext}_{k}^{c}\left(H, \mathbb{G}_{m}\right) \cong \operatorname{Pic}(H)$ (see [8], Corollary 5.7), where $\operatorname{Ext}_{k}^{c}\left(H, \mathbb{G}_{m}\right)$ is the abelian group of isomorphism classes of central extensions of $k$-algebraic groups of $H$ by $\mathbb{G}_{m}$. Given such an extension

$$
1 \rightarrow \mathbb{G}_{m} \rightarrow H_{1} \rightarrow H \rightarrow 1
$$

corresponding to an element $p \in \operatorname{Pic}(H)$, we get a coboundary map in étale cohomology

$$
\partial_{H_{1}}: H^{1}(X, H) \rightarrow H^{2}\left(X, \mathbb{G}_{m}\right),
$$

see [19], IV.4.2.2. This coboundary map fits in the natural exact sequence of pointed sets (see [19], Remark IV.4.2.10)

$$
H^{1}\left(X, H_{1}\right) \rightarrow H^{1}(X, H) \stackrel{\partial_{H_{1}}}{\longrightarrow} H^{2}\left(X, \mathbb{G}_{m}\right)=\operatorname{Br}(X) .
$$

The element $\Delta_{Y / X}(p)$ is defined to be the image of the class $[Y] \in H^{1}(X, H)$ of the torsor $\pi: Y \rightarrow X$ by the map $\partial_{H_{1}}$. This construction defines a map

$$
\Delta_{Y / X}: \operatorname{Pic}(H) \rightarrow \operatorname{Br}(X), \quad p \mapsto \partial_{H_{1}}([Y]),
$$


which is functorial in $X$ and $H$ (this map was denoted by $\delta_{\text {tors }}(Y)$ in [11]).

We can compare the map $\Delta_{Y / X}: \operatorname{Pic}(H) \rightarrow \operatorname{Br}(X)$ with another useful map $\alpha_{Y / X}: H^{1}(k, \hat{H}) \rightarrow \mathrm{Br}_{1}(X)$ defined by the formula

$$
\alpha_{Y / X}(z):=p_{X}^{*}(z) \cup[Y] \in H^{2}\left(X, \mathbb{G}_{m}\right),
$$

where $p_{X}: X \rightarrow \operatorname{Spec}(k)$ is the structure morphism and $[Y] \in H^{1}(X, H)$.

Recall that we have a canonical map $\eta_{H}: H^{1}(k, \hat{H}) \rightarrow \operatorname{Pic}(H)$ coming from Leray's spectral sequence (see for instance [38], Lemma 6.9).

Lemma 2.6. The following diagram is commutative:

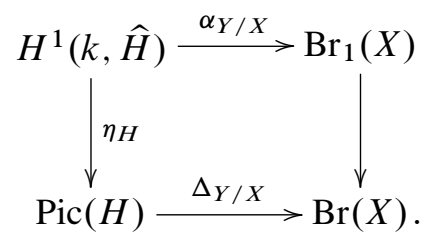

Proof. Define $Z$ to be the quotient of $Y$ by the action of $H^{\text {ssu }}$. Then $Z \rightarrow X$ is a torsor under $H^{\text {tor }}$. By functoriality, and using the isomorphism $\widehat{H^{\text {tor }}} \cong \widehat{H}$, it is sufficient to prove the commutativity of the following diagram:

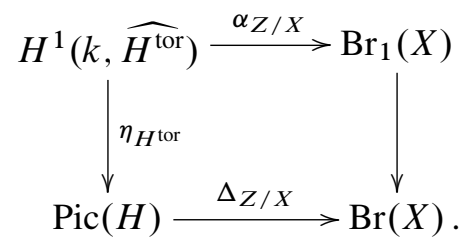

Consider the groups $\operatorname{Ext}_{k}^{n}\left(H^{\text {tor }}, \mathbb{G}_{m}\right)$ in the abelian category of fppf-sheaves over $\operatorname{Spec}(k)$. By [29], Lemmas A.3.1 and A.3.2, we know that the diagram

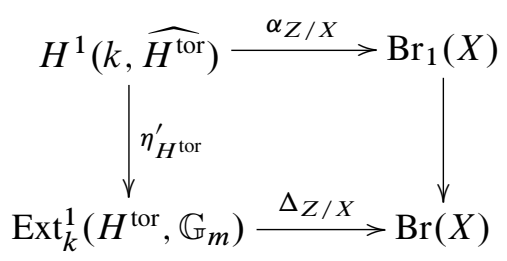

is commutative, where $\eta_{H^{\text {tor }}}^{\prime}: H^{1}\left(k, \widehat{H^{\text {tor }}}\right) \rightarrow \operatorname{Ext}_{k}^{1}\left(H^{\text {tor }}, \mathbb{G}_{m}\right)$ is the edge map from the local to global Ext's spectral sequence

$$
H^{p}\left(k, \operatorname{Ext}_{\bar{k}}^{q}\left(\bar{H}^{\text {tor }}, \mathbb{G}_{m} \bar{k}\right)\right) \Longrightarrow \operatorname{Ext}_{k}^{p+q}\left(H^{\text {tor }}, \mathbb{G}_{m}\right)
$$

(see [1], V.6.1). 
By [35], Proposition 17.5, there exists a canonical map $\operatorname{Ext}_{k}^{1}\left(H^{\text {tor }}, \mathbb{G}_{m}\right) \rightarrow$ $\operatorname{Ext}_{k}^{c}\left(H^{\text {tor }}, \mathbb{G}_{m}\right)$. Composing this map with $c_{H^{\text {tor }}}: \operatorname{Ext}_{k}^{c}\left(H^{\text {tor }}, \mathbb{G}_{m}\right) \rightarrow \operatorname{Pic}\left(H^{\text {tor }}\right)$ (used in the construction of $\left.\Delta_{Z / X}\right)$, we get a map $c_{H^{\text {tor }}}^{\prime}$ : $\operatorname{Ext}_{k}^{1}\left(H^{\text {tor }}, \mathbb{G}_{m}\right) \rightarrow \operatorname{Pic}\left(H^{\text {tor }}\right)$.

It is now sufficient to prove that the diagram

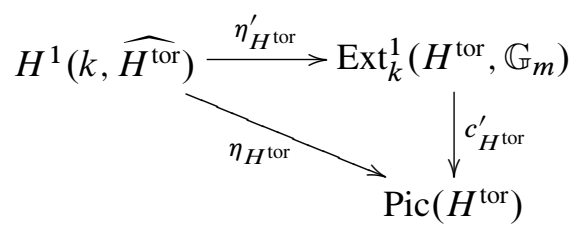

is commutative.

The natural transformation $\operatorname{Hom}_{\bar{k} \text {-groups }}\left(\bar{H}^{\text {tor }},(.)_{\bar{k}}\right) \stackrel{\tau}{\rightarrow} H^{0}\left(\bar{H}^{\text {tor }},(.)_{\bar{k}}\right)$ of functors from the category of fppf-sheaves over $\operatorname{Spec}(k)$ to the category of $\operatorname{Gal}(\bar{k} / k)$ modules induces a morphism of spectral sequences

$$
\begin{aligned}
\left(H^{p}\left(k, \operatorname{Ext}_{\bar{k}}^{q}\left(\bar{H}^{\text {tor }}, \mathbb{G}_{m} \bar{k}\right)\right)\right. & \left.\Longrightarrow \operatorname{Ext}_{k}^{p+q}\left(H^{\text {tor }}, \mathbb{G}_{m}\right)\right) \\
& \stackrel{\tau}{\longrightarrow}\left(H^{p}\left(k, H^{q}\left(\bar{H}^{\text {tor }}, \mathbb{G}_{m \bar{k}}\right)\right) \Longrightarrow H^{p+q}\left(H^{\text {tor }}, \mathbb{G}_{m}\right)\right)
\end{aligned}
$$

from the local to global Ext's spectral sequence to Leray's spectral sequence. This morphism implies that the induced diagram between edge maps

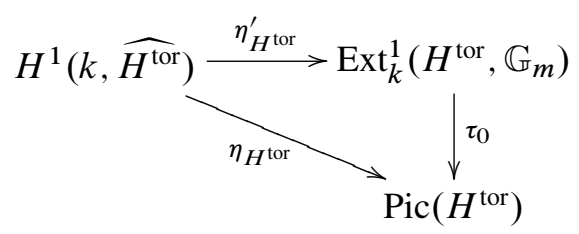

is commutative. We need to prove that the map $\tau_{0}$ induced by $\tau$ coincides with the map $c_{H^{\text {tor }}}^{\prime}: \operatorname{Ext}_{1}^{c}\left(H^{\text {tor }}, \mathbb{G}_{m}\right) \rightarrow \operatorname{Pic}\left(H^{\text {tor }}\right)$. Let

$$
0 \rightarrow \mathbb{G}_{m k} \rightarrow I \rightarrow Q \rightarrow 0
$$

be an exact sequence of fppf-sheaves on $\operatorname{Spec}(k)$ such that $I$ is injective. Then the long exact sequences associated to the functors $H^{0}\left(H^{\text {tor }},.\right)$ and $\operatorname{Hom}_{k}\left(H^{\text {tor }},.\right)$ give rise to the following commutative diagram

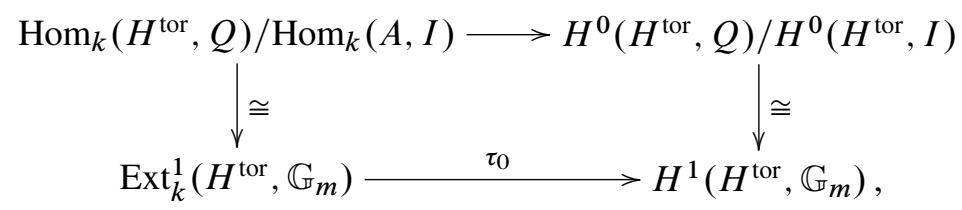

where the vertical maps are the coboundary maps and the horizontal ones are induced by $\tau$. With this diagram, it is clear that the image by $\tau_{0}$ of a given group extension is 
the same as the image of this extension by the map $c_{H^{\text {tor }}}^{\prime}$, which concludes the proof.

Remark 2.7. In particular, if $H=T$ is a $k$-torus, then the map $\eta_{T}: H^{1}(k, \widehat{T}) \stackrel{\cong}{\rightarrow}$ $\operatorname{Pic}(T)$ is an isomorphism, and we see that the map $\Delta_{Y / X}$ coincides with the map $\alpha_{Y / X}: H^{1}(k, \widehat{T}) \rightarrow \mathrm{Br}_{1}(X)$ defined by $z \mapsto p_{X}^{*}(z) \cup[Y]$.

The goal of the following theorem is to give an equivalent of Sansuc's exact sequence (6) with the map $\Delta_{Y / X}^{\prime}$ replaced by the map $\Delta_{Y / X}$. It will be very important in the following.

Theorem 2.8. Let $k$ be a field of characteristic zero. Let $H$ a connected linear $k$ group, $X$ a smooth $k$-variety and $\pi: Y \stackrel{H}{\rightarrow} X$ a (left) torsor under $H$. Then we have a commutative diagram with exact rows, functorial in $(X, Y, \pi, H)$ :

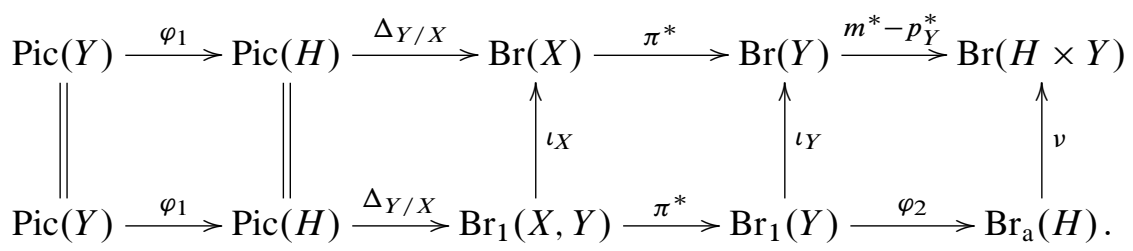

Here $m: H \times Y \rightarrow Y$ denotes the left action of $H$ on $Y$, the homomorphism $\Delta_{Y / X}: \operatorname{Pic}(H) \rightarrow \operatorname{Br}(X)$ is the map of [11], see 2.5 above, the homomorphisms $\varphi_{1}$ and $\varphi_{2}$ are defined in [38] (6.4.1) (or see (4)), the homomorphisms $\iota_{X}$ and $\iota_{Y}$ are the inclusion maps, and the injective homomorphism $v$ is given as the composite of the following natural injective maps:

$$
\mathrm{Br}_{\mathrm{a}}(H) \rightarrow \mathrm{Br}_{\mathrm{a}}(H) \oplus \mathrm{Br}_{1}(Y) \stackrel{\cong}{\longrightarrow} \operatorname{Br}_{1}(H \times Y) \hookrightarrow \operatorname{Br}(H \times Y) .
$$

In particular, if $Y(k) \neq \emptyset$ and $y \in Y(k), x=\pi(y)$, then the maps $\varphi_{i}$ are induced by the map $i_{y}: H \rightarrow Y$ defined by $h \mapsto h . y$, and we have an exact sequence

$$
\operatorname{Pic}(Y) \stackrel{i_{y}^{*}}{\longrightarrow} \operatorname{Pic}(H) \stackrel{\Delta_{Y / X}}{\longrightarrow} \operatorname{Br}_{1, x}(X, Y) \stackrel{\pi^{*}}{\longrightarrow} \operatorname{Br}_{1, y}(Y) \stackrel{i_{y}^{*}}{\longrightarrow} \operatorname{Br}_{1, e}(H) .
$$

Remark 2.9. Recall that the exact sequences (8) and (9) can be extended to the left by

$$
0 \rightarrow k[X]^{*} / k^{*} \rightarrow k[Y]^{*} / k^{*} \rightarrow \hat{H}(k) \rightarrow \operatorname{Pic}(X) \rightarrow \operatorname{Pic}(Y)
$$

(see [38], Proposition 6.10).

Corollary 2.10. Let $k$ be a field of characteristic zero. Let $T$ be a $k$-torus, $X$ a smooth $k$-variety and $\pi: Y \stackrel{T}{\rightarrow} X$ a (left) torsor under $T$. Then we have an exact sequence

$$
\operatorname{Pic}(Y) \stackrel{\varphi_{1}}{\longrightarrow} \operatorname{Pic}(T) \stackrel{\Delta_{Y / X}}{\longrightarrow} \operatorname{Br}_{1}(X) \stackrel{\pi^{*}}{\longrightarrow} \operatorname{Br}_{1}(Y) \stackrel{\varphi_{2}}{\longrightarrow} \operatorname{Br}_{\mathrm{a}}(T) .
$$


Proof. It is a direct application of Theorem 2.8, using $\operatorname{Pic}(\bar{T})=0$.

Remark 2.11. This corollary compares the algebraic Brauer groups of $X$ and $Y$. Concerning the transcendental part of those groups, Theorem 2.8 can be used to study the injectivity of the map $\operatorname{Br}(X) \rightarrow \operatorname{Br}(Y)$. We cannot describe easily the image of this map in general. However, Harari and Skorobogatov studied this map in particular cases (universal torsors for instance): see [23], Theorems 1.6 and 1.7.

Corollary 2.12 (cf. [38], Corollary 6.11). Let

$$
1 \rightarrow G^{\prime} \stackrel{i}{\longrightarrow} G \stackrel{j}{\longrightarrow} G^{\prime \prime} \rightarrow 1
$$

be an exact sequence of connected algebraic groups over a field $k$ of characteristic zero. Assume that $G^{\prime}$ is linear. Then there is a commutative diagram with exact rows

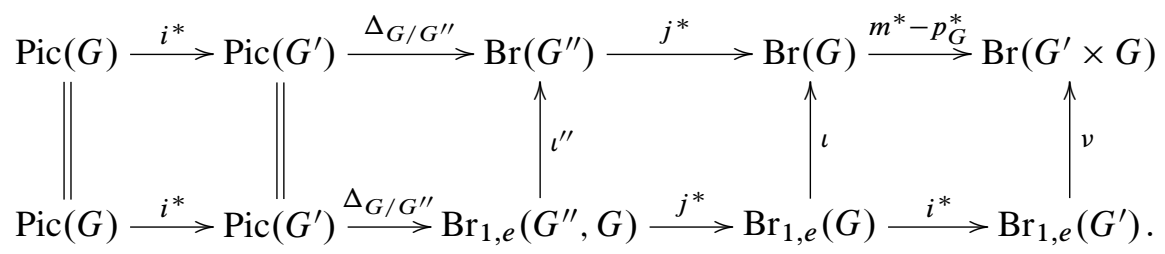

Here $p_{G}: G^{\prime} \times G \rightarrow G$ is the projection map, the map $m: G^{\prime} \times G \rightarrow G$ is defined by $m\left(g^{\prime}, g\right):=i\left(g^{\prime}\right) \cdot g$ (where the product denotes the group law in $\left.G\right), \iota^{\prime \prime}$ and $\iota$ are the inclusion homomorphisms, and the injective homomorphism $v$ is defined as in Theorem 2.8.

If the homomorphism $\operatorname{Pic}(\bar{G}) \rightarrow \operatorname{Pic}\left(\bar{G}^{\prime}\right)$ is surjective (e.g. when $G^{\prime}$ is a k-torus, or when $G^{\prime s s}$ is simply connected, or when all the three groups $G^{\prime}, G$ and $G^{\prime \prime}$ are linear $)$, then $\operatorname{Br}_{1, e}\left(G^{\prime \prime}, G\right)=\operatorname{Br}_{1, e}\left(G^{\prime \prime}\right)$, and we have a commutative diagram with exact rows:

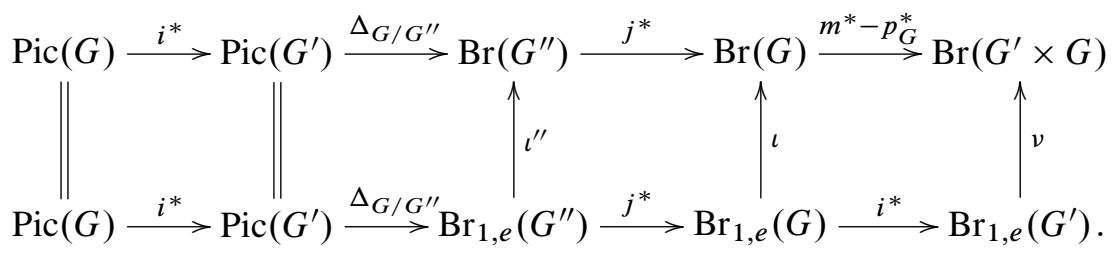

Proof. The short exact sequence of algebraic groups defines a structure of (left) $G^{\prime \prime}$ torsor under $G^{\prime}$ on $G$ ( $G^{\prime}$ acts on $G$ by left translations). Now from the diagram with exact rows (8) we obtain diagram (10), which differs from diagram (11) by the middle term in the bottom row.

From diagram (8) we obtain an exact sequence

$$
\operatorname{Pic}(\bar{G}) \stackrel{i^{*}}{\rightarrow} \operatorname{Pic}\left(\overline{G^{\prime}}\right) \stackrel{\Delta_{G / G^{\prime \prime}}}{\longrightarrow} \operatorname{Br}\left(\overline{G^{\prime \prime}}\right) \stackrel{j^{*}}{\longrightarrow} \operatorname{Br}(\bar{G}) .
$$


If the homomorphism $i^{*}: \operatorname{Pic}(\bar{G}) \rightarrow \operatorname{Pic}\left(\bar{G}^{\prime}\right)$ is surjective, then the homomorphism $j^{*}: \operatorname{Br}\left(\overline{G^{\prime \prime}}\right) \rightarrow \operatorname{Br}(\bar{G})$ is injective, hence $\operatorname{Br}_{1, e}\left(G^{\prime \prime}, G\right)=\operatorname{Br}_{1, e}\left(G^{\prime \prime}\right)$, and we obtain diagram (11) from diagram (10).

If $G^{\prime}$ is a $k$-torus or if $G^{\prime \text { ss }}$ is simply connected, then $\operatorname{Pic}\left(\bar{G}^{\prime}\right)=0$, and the homomorphism $\operatorname{Pic}(\bar{G}) \rightarrow \operatorname{Pic}\left(\bar{G}^{\prime}\right)$ is clearly surjective. If all the three groups $G^{\prime}$, $G$ and $G^{\prime \prime}$ are linear, then again the homomorphism $\operatorname{Pic}(\bar{G}) \rightarrow \operatorname{Pic}\left(\overline{G^{\prime}}\right)$ is surjective, see [38], proof of Corollary 6.11, p. 44.

For the proof of Theorem 2.8 we need a crucial lemma.

Lemma 2.13. Let $k$ be a field of characteristic zero. Let $H$ a connected linear $k$-group, $X$ a smooth $k$-variety and $\pi: Y \stackrel{H}{\rightarrow} X$ a (left) torsor under $H$. Let $\tau: Z \stackrel{\mathbb{G}_{m}}{\longrightarrow} Y$ be a torsor under $\mathbb{G}_{m}$. Then there exists a central extension of algebraic k-groups

$$
1 \rightarrow \mathbb{G}_{m} \rightarrow H_{1} \rightarrow H \rightarrow 1
$$

and a left action $H_{1} \times Z \rightarrow Z$, extending the action of $\mathbb{G}_{m}$ on $Z$ and compatible with the action of $H$ on $Y$. This action makes $Z \rightarrow X$ into a torsor under $H_{1}$. Moreover, the class of such an extension $H_{1}$ in the group $\operatorname{Ext}_{k}^{\mathrm{c}}\left(H, \mathbb{G}_{m}\right)$ is uniquely determined, namely $\left[H_{1}\right]=\varphi_{1}([Z]) \in \operatorname{Ext}_{k}^{\mathrm{c}}\left(H, \mathbb{G}_{m}\right)=\operatorname{Pic}(H)$.

Remark 2.14. In [24], Harari and Skorobogatov studied this question of composition of torsors. Their results (see Theorem 2.2 and Proposition 2.5 in [24]) deal with torsors under multiplicative groups and not only under $\mathbb{G}_{m}$ as here, but they require additional assumptions concerning the type of the torsor and on invertible functions on the varieties. Those additional assumptions are not satisfied in our context.

Proof. Let $p_{H}: H \times Y \rightarrow H$ and $p_{Y}: H \times Y \rightarrow Y$ denote the two projections. Let

$$
1 \rightarrow \mathbb{G}_{m} \rightarrow H_{1} \rightarrow H \rightarrow 1
$$

be a central extension such that its class in $\operatorname{Ext}_{k}^{\mathrm{c}}\left(H, \mathbb{G}_{m}\right)=\operatorname{Pic}(H)$ is exactly $\varphi_{1}([Z])$.

In this setting, Lemma 2.3 implies that

$$
m^{*}[Z]=p_{H}^{*}\left[H_{1}\right]+p_{Y}^{*}[Z]
$$

Formula (13) means that the push-forward of the torsor

$$
H_{1} \times Z \stackrel{\mathbb{G}_{m} \times \mathbb{G}_{m}}{\longrightarrow} H \times Y
$$

by the group law homomorphism $\mathbb{G}_{m} \times \mathbb{G}_{m} \rightarrow \mathbb{G}_{m}$ is isomorphic (as a $H \times Y$-torsor under $\mathbb{G}_{m}$ ) to the pullback $m^{*} Z$ of the torsor $Z \rightarrow Y$ by the map $m: H \times Y \rightarrow Y$. 
In particular, we get the following commutative diagram:

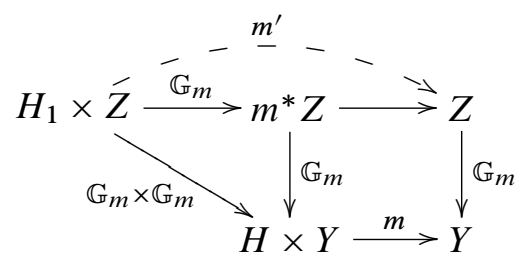

where $m^{\prime}$ is defined to be the composite of the two upper horizontal maps. The situation is very similar to that in the proof of Theorem 5.6 in [8]: the map $m^{\prime}$ fits into commutative diagram (14) and for all $t_{1}, t_{2} \in \mathbb{G}_{m}$ and all $h_{1} \in H_{1}, z \in Z$, we have

$$
m^{\prime}\left(t_{1} \cdot h_{1}, t_{2} \cdot z\right)=t_{1} t_{2} \cdot m^{\prime}\left(h_{1}, z\right) .
$$

We want to use $m^{\prime}$ to define a group action of $H_{1}$ on $Z$. Formula (15) implies that the morphism $m^{\prime}(e,):. z \mapsto m^{\prime}(e, z)$ is an automorphism of the $Y$-torsor $Z$, and we can define a map $m^{\prime \prime}: H_{1} \times Z \rightarrow Z$ to be the composition

$$
m^{\prime \prime}:=m^{\prime}(e, .)^{-1} \circ m^{\prime}: H_{1} \times Z \rightarrow Z .
$$

Then we get a commutative diagram

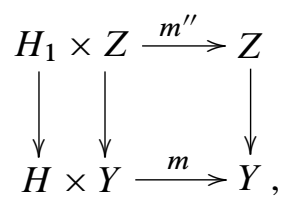

where the map $m^{\prime \prime}$ still satisfies formula (15) and now, for all $z \in Z$, we have

$$
m^{\prime \prime}(e, z)=z
$$

We wish to prove that $m^{\prime \prime}$ is a left group action of $H_{1}$ on $Z$.

Since $m: H \times Y \rightarrow Y$ is a left action, we have

$$
\tau\left(m^{\prime \prime}\left(h_{1} h_{2}, z\right)\right)=\tau\left(m^{\prime \prime}\left(h_{1}, m^{\prime \prime}\left(h_{2}, z\right)\right)\right) \quad \text { for } h_{1}, h_{2} \in H_{1}, z \in Z,
$$

where $\tau: Z \rightarrow Y$ is the canonical map. Since $\tau: Z \rightarrow Y$ is a torsor under $\mathbb{G}_{m}$, there is a canonical map

$$
Z \times{ }_{Y} Z \rightarrow \mathbb{G}_{m}, \quad\left(z_{1}, z_{2}\right) \mapsto z_{1} z_{2}^{-1}
$$

We obtain a morphism of $k$-varieties

$$
\varphi: H_{1} \times H_{1} \times Z \rightarrow \mathbb{G}_{m}, \quad\left(h_{1}, h_{2}, z\right) \mapsto \varphi_{z}\left(h_{1}, h_{2}\right),
$$


such that

$$
m^{\prime \prime}\left(h_{1} h_{2}, z\right)=\varphi_{z}\left(h_{1}, h_{2}\right) \cdot m^{\prime \prime}\left(h_{1}, m^{\prime \prime}\left(h_{2}, z\right)\right) \quad \text { for } h_{1}, h_{2} \in H_{1}, z \in Z .
$$

Then (17) implies that

$$
\varphi_{z}(h, e)=1 \quad \text { and } \quad \varphi_{z}(e, h)=1 .
$$

By Rosenlicht's lemma (see [37], Theorem 3, see also [38], Lemma 6.5), the map $\varphi$ has to be trivial, i.e. $\varphi_{z}\left(h_{1}, h_{2}\right)=1$ for all $z, h_{1}, h_{2}$. Therefore we have

$$
m^{\prime \prime}\left(h_{1} h_{2}, z\right)=m^{\prime \prime}\left(h_{1}, m^{\prime \prime}\left(h_{2}, z\right)\right) .
$$

Formulas (17) and (18) show that $m^{\prime \prime}$ is a left group action of $H_{1}$ on $Z$. Since $m^{\prime \prime}$ satisfies (15), we have for $t \in \mathbb{G}_{m}, z \in Z$

$$
m^{\prime \prime}(t e, z)=t \cdot m^{\prime \prime}(e, z)=t . z,
$$

hence the action $m^{\prime \prime}$ extends the action of $\mathbb{G}_{m}$ on $Z$. From diagram (16) with $m^{\prime \prime}$ instead of $m^{\prime}$ we see that the action $m^{\prime \prime}$ induces the action $m$ of $H$ on $Y$.

Consider the following commutative diagram (see (16)):

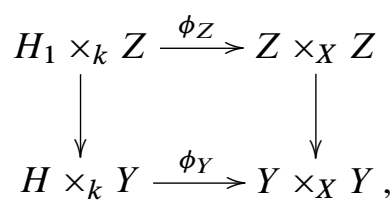

where $\phi_{Z}\left(h_{1}, z\right):=\left(m^{\prime \prime}\left(h_{1}, z\right), z\right), \phi_{Y}(h, y):=(m(h, y), y)$, and the unnamed morphisms are the natural ones. Since $Y \rightarrow X$ is a torsor under $H$, the morphism $\phi_{Y}$ is an isomorphism. The group $\mathbb{G}_{m} \times \mathbb{G}_{m}$ acts on $H_{1} \times_{k} Z$ via $\left(t_{1}, t_{2}\right) .\left(h_{1}, z\right):=$ $\left(t_{1} h_{1}, t_{2} . z\right)$, making $H_{1} \times_{k} Z \rightarrow H \times_{k} Y$ into a torsor under $\mathbb{G}_{m} \times \mathbb{G}_{m}$. We define an action of $\mathbb{G}_{m} \times \mathbb{G}_{m}$ on $Z \times_{X} Z$ by

$$
\left(t_{1}, t_{2}\right) \cdot\left(z_{1}, z_{2}\right):=\left(\left(t_{1} t_{2}\right) \cdot z_{1}, t_{2} \cdot z_{2}\right),
$$

then $Z \times_{X} Z \rightarrow Y \times_{X} Y$ is a torsor under $\mathbb{G}_{m} \times \mathbb{G}_{m}$. By formula (15) the map $\phi_{Z}$ in (19) is a morphism of torsors under $\mathbb{G}_{m} \times \mathbb{G}_{m}$ compatible with the isomorphism $\phi_{Y}$ of $k$-varieties. Therefore the map $\phi_{Z}$ is an isomorphism of $k$-varieties, which proves that the action $m^{\prime \prime}$ makes $Z \rightarrow X$ into a torsor under $H_{1}$.

Let us prove the uniqueness of the class of the extension $H_{1}$. If $H_{2}$ is a central extension of $H$ that satisfies the conditions of the lemma, then the analogues of diagram (16) and formula (15) with $H_{2}$ instead of $H_{1}$ define an isomorphism of $H \times Y$-torsors under $\mathbb{G}_{m}$ between the push-forward of $H_{2} \times Z$ by the morphism $\mathbb{G}_{m} \times \mathbb{G}_{m} \rightarrow \mathbb{G}_{m}$ and the torsor $m^{*} Z$. Therefore, we get

$$
m^{*}[Z]=p_{H}^{*}\left[H_{2}\right]+p_{Y}^{*}[Z] .
$$


Comparing with (13), we see that $p_{H}^{*}\left[H_{2}\right]=p_{H}^{*}\left[H_{1}\right]$. Since $p_{H}^{*}+p_{Y}^{*}: \operatorname{Pic}(H) \oplus$ $\operatorname{Pic}(Y) \rightarrow \operatorname{Pic}(H \times Y)$ is an isomorphism, we see that $p_{H}^{*}: \operatorname{Pic}(H) \rightarrow \operatorname{Pic}(H \times Y)$ is an embedding, hence $\left[H_{2}\right]=\left[H_{1}\right]$, which completes the proof of Lemma 2.13.

2.15. Proof of Theorem 2.8: top row of the diagram. First we prove that the top row in diagram (8) is a complex. Let $p \in \operatorname{Pic}(Y)$ and let us prove that $\Delta_{Y / X}\left(\varphi_{1}(p)\right)=0$. Let $Z \rightarrow Y$ be a torsor under $\mathbb{G}_{m}$ such that $[Z]=p \in \operatorname{Pic}(Y)$. Let $p^{\prime}:=\varphi_{1}(p) \in$ $\operatorname{Pic}(H)$, and $1 \rightarrow \mathbb{G}_{m} \rightarrow H_{1} \rightarrow H \rightarrow 1$ be a central extension of $H$ by $\mathbb{G}_{m}$ corresponding to $p^{\prime}$ via the isomorphism $\operatorname{Ext}_{k}^{c}\left(H, \mathbb{G}_{m}\right) \cong \operatorname{Pic}(H)$. Then $\Delta_{Y / X}\left(p^{\prime}\right)$ is equal (by definition) to $\partial_{H_{1}}([Y]) \in H^{2}\left(X, \mathbb{G}_{m}\right)$, where $\partial_{H_{1}}: H^{1}(X, H) \rightarrow$ $H^{2}\left(X, \mathbb{G}_{m}\right)$ is the coboundary map coming from the extension $H_{1}$, and $[Y]$ is the class of the torsor $Y \rightarrow X$ in $H^{1}(X, H)$.

Lemma 2.13 implies that the class $[Y] \in H^{1}(X, H)$ is in the image of the map $H^{1}\left(X, H_{1}\right) \rightarrow H^{1}(X, H)$. From exact sequence (7) we see that the class $\partial_{H_{1}}([Y])$ is trivial in $H^{2}\left(X, \mathbb{G}_{m}\right)$, i.e. $\Delta_{Y / X}\left(p^{\prime}\right)=\partial_{H_{1}}([Y])=0 \in H^{2}\left(X, \mathbb{G}_{m}\right)$, hence $\operatorname{Pic}(Y) \stackrel{j^{*}}{\longrightarrow} \operatorname{Pic}(H) \stackrel{\Delta_{Y / X}}{\longrightarrow} \operatorname{Br}(X)$ is a complex.

Let $p \in \operatorname{Pic}(H)$, and let us prove that $\pi^{*}\left(\Delta_{Y / X}(p)\right)=0$. The element $p$ corresponds to the class of an extension $1 \rightarrow \mathbb{G}_{m} \rightarrow H_{1} \rightarrow H \rightarrow 1$, and we have $\Delta_{Y / X}(p)=\partial_{H_{1}}([Y])$. We have a commutative diagram

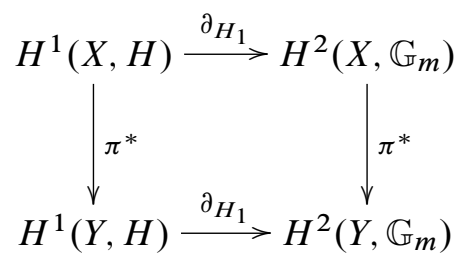

so that $\pi^{*}\left(\Delta_{Y / X}(p)\right)=\pi^{*}\left(\partial_{H_{1}}([Y])\right)=\partial_{H_{1}}\left(\pi^{*}[Y]\right)$. The torsor $\pi^{*}[Y]$ is trivial in the set $H^{1}(Y, H)$, hence $\pi^{*}\left(\Delta_{Y / X}(p)\right)=0$. Consequently the top row of diagram (8) is a complex.

Let us prove that the top row of diagram (8) is exact. For the exactness at the term $\operatorname{Br}(Y)$, see Proposition 2.4. So it remains to prove the exactness of the top row at $\operatorname{Pic}(H)$ and at $\operatorname{Br}(X)$.

Let $p \in \operatorname{Pic}(H)$ be such that $\Delta_{Y / X}(p)=0$. Such a $p$ corresponds to the class of an extension $1 \rightarrow \mathbb{G}_{m} \rightarrow H_{1} \stackrel{q}{\rightarrow} H \rightarrow 1$ such that $\partial_{H_{1}}([Y])=0$. From exact sequence (7) we see that there exists an $X$-torsor $Z \stackrel{H_{1}}{\longrightarrow} X$ under $H_{1}$, with an $H_{1}$ equivariant map $Z \rightarrow Y$, making $Z \rightarrow Y$ into a torsor under $\mathbb{G}_{m}$. Then by the uniqueness part of Lemma 2.13 we know that the class of $Z \rightarrow Y$ in $\operatorname{Pic}(Y)$ maps to the class of $H_{1}$ in $\operatorname{Pic}(H)$, i.e. $\varphi_{1}([Z])=p$, which proves the exactness of the top row of diagram (8) at $\operatorname{Pic}(H)$.

Let us now prove the exactness of the top row of (8) at $\operatorname{Br}(X)$. 
Assume first that the $k$-variety $X$ is quasi-projective. Let $A \in \operatorname{Br}(X)$ such that $\pi^{*} A=0 \in \operatorname{Br}(Y)$. By a theorem of Gabber also proven by de Jong (see [12]), we know that there exists a positive integer $n$ and an $X$-torsor $Z \rightarrow X$ under PGL $_{n}$ such that $-A$ is the image of the class of $Z$ in $H^{1}\left(X, \mathrm{PGL}_{n}\right)$ by the coboundary map $H^{1}\left(X, \mathrm{PGL}_{n}\right) \stackrel{\partial_{\mathrm{GL} n}}{\longrightarrow} H^{2}\left(X, \mathbb{G}_{m}\right)$. Let $W$ denote the product $Y \times_{X} Z$. From the commutative diagram with exact rows

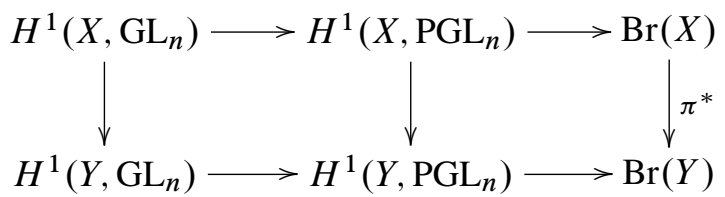

we see that the assumption $\pi^{*} A=0$ implies that the torsor $W \stackrel{\mathrm{PGL}_{n}}{\longrightarrow} Y$ is dominated by some $Y$-torsor under $\mathrm{GL}_{n}$, i.e. there exists a torsor $V \stackrel{\mathrm{GL}_{n}}{\longrightarrow} Y$ and a morphism of $Y$-torsors $V \rightarrow W$ compatible with the quotient morphism $\mathrm{GL}_{n} \rightarrow \mathrm{PGL}_{n}$. We have the following picture:

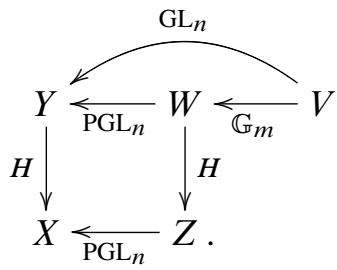

Since $W \rightarrow X$ is a torsor under the connected linear group $H \times \mathrm{PGL}_{n}$, we can apply Lemma 2.13 to get a central extension

$$
1 \rightarrow \mathbb{G}_{m} \rightarrow L \rightarrow H \times \mathrm{PGL}_{n} \rightarrow 1
$$

and a structure of $X$-torsor under $L$ on $V \rightarrow X$, compatible with the action of $H \times \mathrm{PGL}_{n}$ on $W$. In particular, the natural injections of $H$ and $\mathrm{PGL}_{n}$ into $H \times \mathrm{PGL}_{n}$ define two central extensions obtained by pulling back the extension (20):

$$
\begin{gathered}
1 \rightarrow \mathbb{G}_{m} \rightarrow L_{H} \rightarrow H \rightarrow 1, \\
1 \rightarrow \mathbb{G}_{m} \rightarrow L_{\mathrm{PGL}_{n}} \rightarrow \mathrm{PGL}_{n} \rightarrow 1 .
\end{gathered}
$$

Since $\mathrm{PGL}_{n}$ acts trivially on $Y$, the action of $L_{\mathrm{PGL}_{n}}$ (as a subgroup of $L$ ) on $V$ defines a commutative diagram

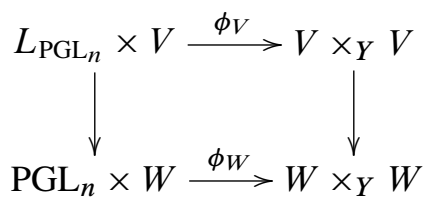


where $\phi_{V}(l, v):=(l . v, v), \phi_{W}(p, w):=(p . w, w)$, and the vertical maps are the natural ones. We see easily that $\phi_{V}$ is an isomorphism, hence $V \rightarrow Y$ is a torsor under $L_{\mathrm{PGL}}$. This action of $L_{\mathrm{PGL}}$ extends the action of $\mathbb{G}_{m}$ on $V$ above $W$, and is compatible with the action of $\mathrm{PGL}_{n}$ on $W$ above $Y$ via the extension (22) and the map $V \rightarrow W$. Therefore, the unicity result in Lemma 2.13 implies that exact sequence (22) is equivalent to the usual extension $1 \rightarrow \mathbb{G}_{m} \rightarrow \mathrm{GL}_{n} \rightarrow \mathrm{PGL}_{n} \rightarrow 1$, and in particular that $\partial_{L_{\mathrm{PGL} n}}([Z])=\partial_{\mathrm{GL}_{n}}([Z]) \in H^{2}\left(X, \mathbb{G}_{m}\right)$.

Consider the direct product of the exact sequences (21) and (22):

$$
1 \rightarrow \mathbb{G}_{m} \times \mathbb{G}_{m} \rightarrow L_{H} \times L_{\mathrm{PGL}_{n}} \rightarrow H \times \mathrm{PGL}_{n} \rightarrow 1 .
$$

Define the morphism $\mu: L_{H} \times L_{\mathrm{PGL}_{n}} \rightarrow L$ by $\mu\left(l, l^{\prime}\right):=l . l^{\prime}$, where the product is taken inside the group $L$. By definition of $L_{H}$ and $L_{\mathrm{PGL}_{n}}$, we see that the image of the commutator morphism $c: L_{H} \times L_{\mathrm{PGL}_{n}} \rightarrow L$ defined by $c\left(l, l^{\prime}\right):=l . l^{\prime} \cdot l^{-1} . l^{-1}$ is contained in the central subgroup $\mathbb{G}_{m}$ of $L$. Therefore, since $c\left(e, l^{\prime}\right)=c(l, e)=1$, Rosenlicht's lemma implies that $c\left(l, l^{\prime}\right)=1$ for all $\left(l, l^{\prime}\right) \in L_{H} \times L_{\mathrm{PGL}_{n}}$. Hence the morphism $\mu$ is a group homomorphism, and the following diagram is commutative with exact rows:

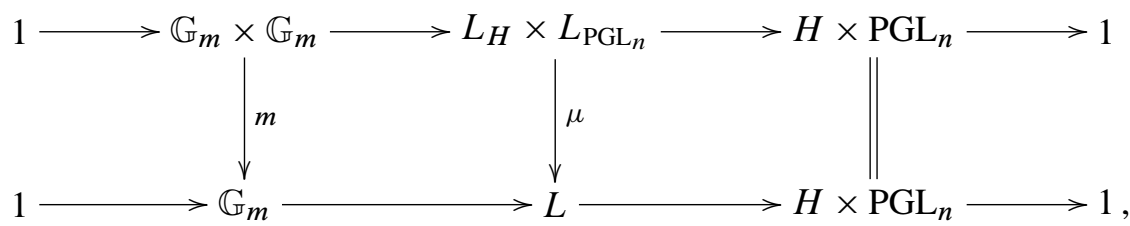

where $m: \mathbb{G}_{m} \times \mathbb{G}_{m} \rightarrow \mathbb{G}_{m}$ is the group law. Therefore we get a commutative diagram of coboundary maps

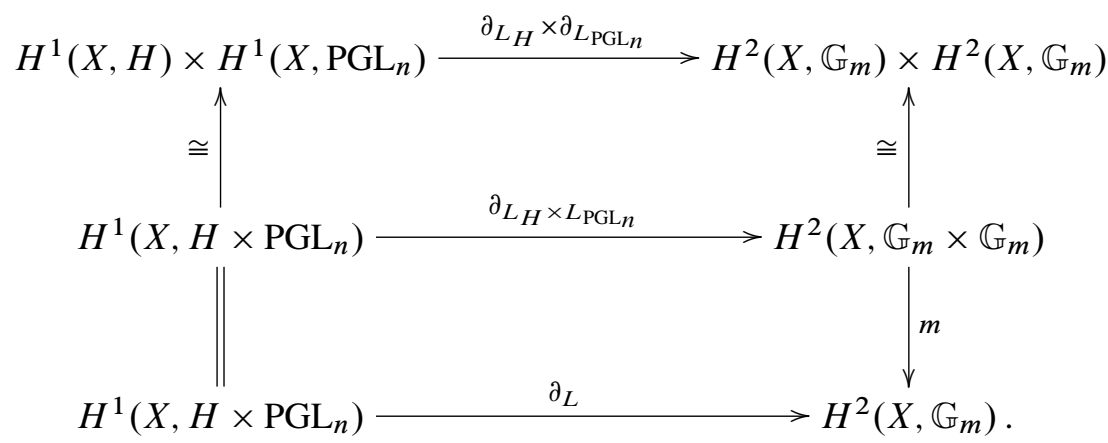

In particular, this diagram implies that

$$
\partial_{L}([W])=\partial_{L_{H}}([Y])+\partial_{L_{\mathrm{PGL} n}}([Z])
$$

in $H^{2}\left(X, \mathbb{G}_{m}\right)$. Since $W \stackrel{H \times \mathrm{PGL}_{n}}{\longrightarrow} X$ is dominated by the $X$-torsor $V \stackrel{L}{\rightarrow} X$, we know that $\partial_{L}([W])=0$, therefore the above formula implies that

$$
\partial_{L_{H}}([Y])=-\partial_{L_{\mathrm{PGL} n}}([Z])=A \in \operatorname{Br}(X),
$$


i.e. $A=\Delta_{Y / X}\left(\left[L_{H}\right]\right)$, with $\left[L_{H}\right] \in \operatorname{Ext}_{k}^{c}\left(H, \mathbb{G}_{m}\right) \cong \operatorname{Pic}(H)$. Hence the first row of diagram (8) is exact under the assumption that $X$ is quasi-projective.

Let us now deduce the general case: $X$ is not supposed to be quasi-projective anymore. By Nagata's theorem (see [32]) we know that there exists a proper $k$ variety $Z$ and an open immersion of $k$-varieties $X \rightarrow Z$. By Chow's lemma (see [15], II.5.6.1 and II.5.6.2, or [39], Chapter VI, §2.1), there exists a projective $k$ variety $Z^{\prime}$ and a projective, surjective birational morphism $Z^{\prime} \rightarrow Z$. Moreover, using Hironaka's resolution of singularities (see [27], see also [3] and [17]), there exists a smooth projective $k$-variety $\widetilde{Z}$ and a birational morphism $\widetilde{Z} \rightarrow Z^{\prime}$. Define $X^{\prime}$ to be the fibred product $X^{\prime}:=\widetilde{Z} \times_{Z} X$. Then $X^{\prime}$ is a open subvariety of $\widetilde{Z}$, hence $X^{\prime}$ is a smooth quasi-projective $k$-variety, and the natural map $X^{\prime} \rightarrow X$ is a birational morphism. Define $Y^{\prime}$ to be the product $Y \times_{X} X^{\prime}$. By the quasi-projective case, we know that in the commutative diagram

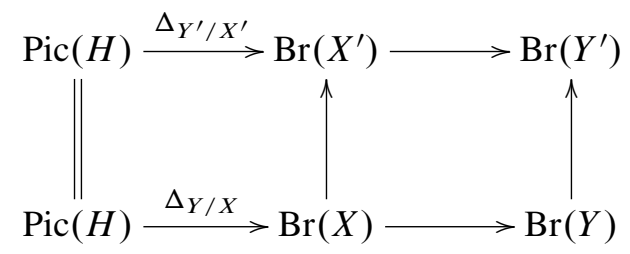

the first row is exact. Since the map $X^{\prime} \rightarrow X$ is a birational morphism, we have a commutative diagram

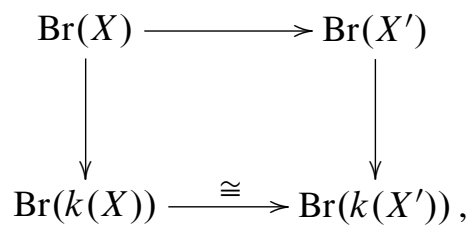

where the bottom horizontal arrow is an isomorphism. Since both $X$ and $X^{\prime}$ are smooth, by [20], II, Corollary 1.8, the vertical arrows are injective. It follows that the homomorphism $\operatorname{Br}(X) \rightarrow \operatorname{Br}\left(X^{\prime}\right)$ is injective. Now a diagram chase in diagram (23) proves the exactness of the second row in that diagram. This completes the proof of the exactness of the top row of diagram (8).

2.16. Proof of Theorem 2.8: the commutativity of the diagram and the exactness of its bottom row. It is clear that all the squares of diagram (8) are commutative except maybe the rightmost square.

By Lemma 2.3, we have for all $\beta_{Y} \in \operatorname{Br}_{1}(Y)$,

$$
m^{*} \beta_{Y}=p_{H}^{*} \varphi_{2}\left(\beta_{Y}\right)+p_{Y}^{*} \beta_{Y} \quad \text { in } \operatorname{Br}_{1}(H \times Y) .
$$

This formula implies immediately that the rightmost square of diagram (8) commutes, hence this diagram is commutative. 
Since the top row of (8) is exact, and the diagram (8) is commutative, it is easy to conclude that the bottom row of (8) is exact.

2.17. Proof of Theorem 2.8: exact sequence (9). We consider the following diagram:

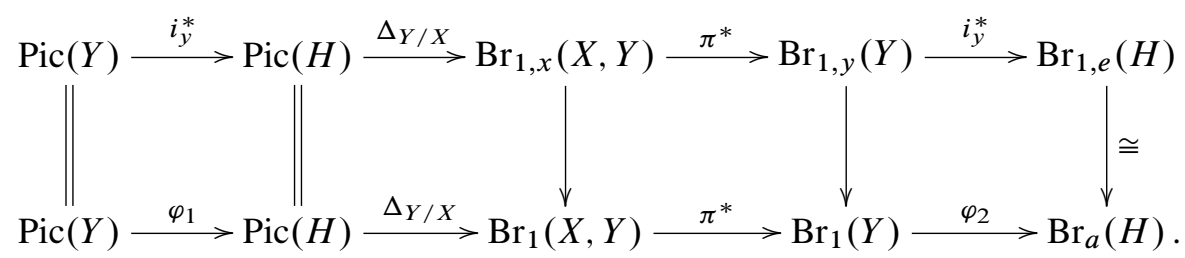

In this diagram the second row is the second row of diagram (8), hence it is exact. In the first row, $\mathrm{Br}_{1, x}(X, Y)$ is a subgroup of $\operatorname{Br}_{1}(X, Y)$ and $\operatorname{Br}_{1, y}(Y)$ is a subgroup of $\mathrm{Br}_{1}(Y)$. We easily check that all the arrows in the first row are well-defined.

This diagram is commutative: for the first and the last squares it is a consequence of Lemma 6.4 of [38], and for the two central squares it is clear. We know that the second row of this diagram is exact. Since all the vertical arrows are injective, we see that the first row is a complex. An easy diagram chase shows that the first row is also exact, i.e. sequence (9) is exact.

\section{Compatibility}

In this section we use Theorem 2.8 to get a compatibility result between the evaluation map and the action of a linear group. We begin with a lemma:

Lemma 3.1. Let $k$ be a field of characteristic 0 and $X, X^{\prime}$ be two smooth $k$-varieties. Let $\pi: Y \stackrel{H}{\longrightarrow} X$ and $\pi^{\prime}: Y^{\prime} \stackrel{H^{\prime}}{\longrightarrow} X^{\prime}$ be two (left) torsors under connected linear $k$ groups $H$ and $H^{\prime}$. The map $Y \times Y^{\prime} \rightarrow X \times X^{\prime}$ is naturally a torsor under $H \times H^{\prime}$. Assume that $\overline{Y^{\prime}}$ is rational and that $Y^{\prime}(k) \neq \emptyset$. Let $y^{\prime} \in Y^{\prime}(k)$ and $x^{\prime}:=\pi^{\prime}\left(y^{\prime}\right)$. Then the homomorphism

$$
p_{X}^{*}+p_{X^{\prime}}^{*}: \operatorname{Br}_{1}(X, Y) \oplus \operatorname{Br}_{1, x^{\prime}}\left(X^{\prime}, Y^{\prime}\right) \rightarrow \operatorname{Br}_{1}\left(X \times X^{\prime}, Y \times Y^{\prime}\right)
$$

is well-defined and is an isomorphism.

Proof. Consider the three exact sequences associated to the torsors $Y \rightarrow X, Y^{\prime} \rightarrow X^{\prime}$ and $Y \times Y^{\prime} \rightarrow X \times X^{\prime}$ (see Theorem 2.8). We get the following commutative diagram 
with exact rows:
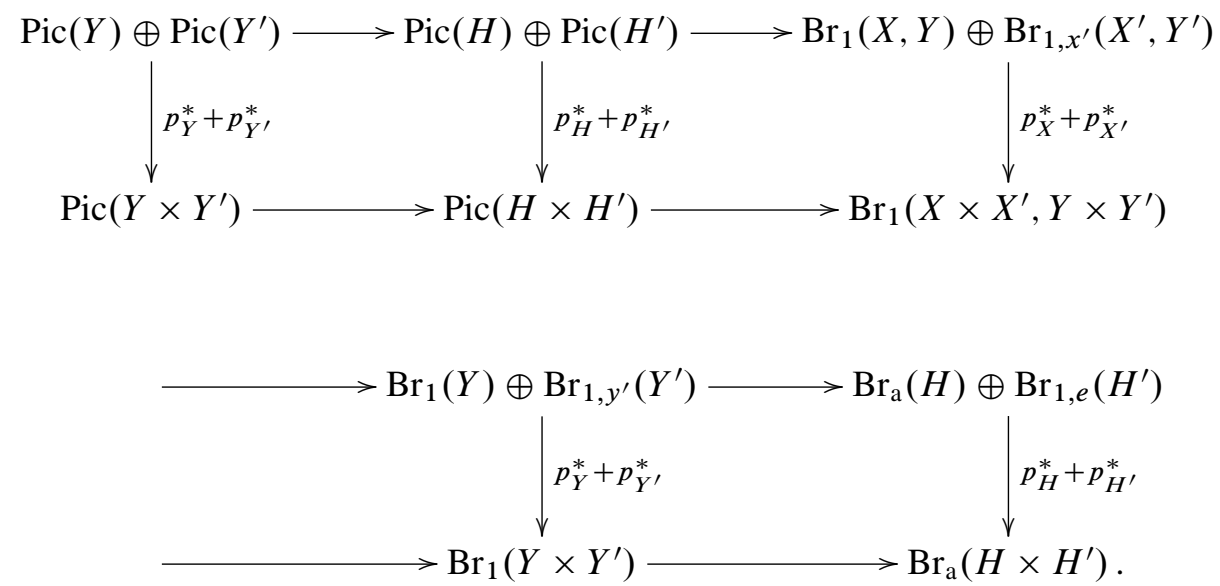

The two first and the two last vertical arrows are isomorphisms by [38], Lemma 6.6, hence the five lemma implies that the central vertical arrow is an isomorphism.

Corollary 3.2. If in Lemma 3.1 we also have $Y(k) \neq \emptyset, y \in Y(k)$ and $x=\pi(y)$, then the homomorphism

$$
p_{X}^{*}+p_{X^{\prime}}^{*}: \mathrm{Br}_{1, x}(X, Y) \oplus \mathrm{Br}_{1, x^{\prime}}\left(X^{\prime}, Y^{\prime}\right) \longrightarrow \operatorname{Br}_{1,\left(x, x^{\prime}\right)}\left(X \times X^{\prime}, Y \times Y^{\prime}\right)
$$

is an isomorphism.

Lemma 3.3 (Compatibility). Let $k$ be a field of characteristic 0 . Let $\pi: Y \rightarrow X$ be a (left) torsor under a connected linear $k$-group $H$. Let $G$ be a connected algebraic $k$-group, acting on the right on $Y$ and $X$ such that $\pi$ is $G$-equivariant. Assume that $Y$ is $\bar{k}$-rational and $Y(k) \neq \emptyset$ and let $y_{0} \in Y(k), x_{0}=\pi\left(y_{0}\right)$. Then for any $b \in \mathrm{Br}_{1, x_{0}}(X, Y), x \in X(k), g \in G(k)$ we have

$$
b(x \cdot g)=b(x)+\pi^{*}(b)\left(y_{0} \cdot g\right) .
$$

Proof. We consider two torsors: $\pi: Y \stackrel{H}{\rightarrow} X$ and $\pi^{\prime}=\mathrm{id}: G \rightarrow G$. Let $m_{X}: X \times$ $G \rightarrow X$ and $m_{Y}: Y \times G \rightarrow Y$ denote the actions of $G$. Since $m_{Y}(y, e)=y$, we see that if $b \in \operatorname{Br}_{y}(X, Y)$, then $m_{X}^{*} b \in \operatorname{Br}_{(y, e)}(X \times G, Y \times G)$. By functoriality of the evaluation map, we have a commutative diagram

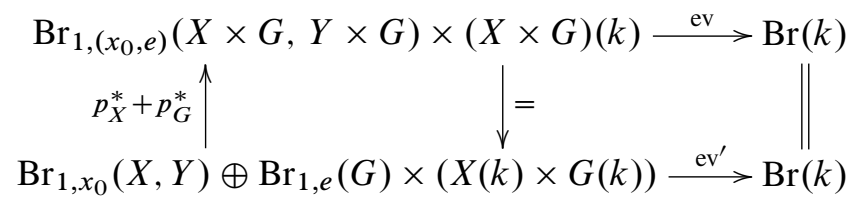


where the first pairing is the evaluation map on $X \times G$ and the second one is the sum of the evaluation maps on $X$ and on $G$, i.e.

$\mathrm{ev}^{\prime}((B, C),(x, g)):=B(x)+C(g) \in \operatorname{Br}(k)$ for $B \in \mathrm{Br}_{1, x_{0}}(X, Y), C \in \mathrm{Br}_{1, e}(G)$.

By Corollary 3.2 the left vertical morphism $p_{X}^{*}+p_{G}^{*}$ is an isomorphism. Therefore we get two natural projections:

$$
\pi_{X}: \mathrm{Br}_{1,\left(x_{0}, e\right)}(X \times G, Y \times G) \rightarrow \mathrm{Br}_{1, x_{0}}(X, Y)
$$

and

$$
\pi_{G}: \mathrm{Br}_{1,\left(x_{0}, e\right)}(X \times G, Y \times G) \rightarrow \operatorname{Br}_{1, e}(G) .
$$

Hence for all $D \in \mathrm{Br}_{1,\left(x_{0}, e\right)}(X \times G, Y \times G)$, we get $D=p_{X}^{*}\left(\pi_{X}(D)\right)+p_{G}^{*}\left(\pi_{G}(D)\right)$, and so, by the commutativity of the diagram, for all $(x, g) \in X(k) \times G(k)$ we have

$$
D((x, g))=\pi_{X}(D)(x)+\pi_{G}(D)(g) .
$$

For $g=e \in G(k)$, this formula implies that

$$
D((x, e))=\pi_{X}(D)(x)
$$

because $\pi_{G}(D)(e)=0$. So, for $b \in \operatorname{Br}_{1, x_{0}}(X, Y), g \in G(k)$ and $x \in X(k)$, we have

$$
\begin{aligned}
b(x . g) & =\left(m^{*} b\right)((x, g)) \\
& =\pi_{X}\left(m^{*} b\right)(x)+\pi_{G}\left(m^{*} b\right)(g) \\
& =\left(m^{*} b\right)((x, e))+\pi_{G}\left(m^{*} b\right)(g)
\end{aligned}
$$

where we write $m$ for $m_{X}$. Since $\left(m^{*} b\right)((x, e))=b(m(x, e))=b(x)$ by functoriality, we have

$$
b(x \cdot g)=b(x)+\pi_{G}\left(m^{*} b\right)(g) .
$$

In the case $x=x_{0}=\pi\left(y_{0}\right)$ we obtain

$$
\left(\pi^{*} b\right)\left(y_{0} \cdot g\right)=b\left(\pi\left(y_{0}\right) \cdot g\right)=b\left(\pi\left(y_{0}\right)\right)+\pi_{G}\left(m^{*} b\right)(g)=\pi_{G}\left(m^{*} b\right)(g),
$$

since $b\left(\pi\left(y_{0}\right)\right)=b\left(x_{0}\right)=0$. Consequently, (26) and (27) give the expected formula, that is:

$$
b(x \cdot g)=b(x)+\left(\pi^{*} b\right)\left(y_{0} \cdot g\right)
$$

Corollary 3.4. Let $X:=H \backslash G$ be a right homogeneous space of a connected linear $k$-group $G$ over a field $k$ of characteristic 0 , where $H \subset G$ is a connected linear $k$-subgroup. Then for all $b \in \mathrm{Br}_{1, x_{0}}(X, G), x \in X(k), g \in G(k)$ we have

$$
b(x . g)=b(x)+\pi^{*} b(g) \text {, }
$$

where $\pi: G \rightarrow X$ is the quotient map and $x_{0}=\pi(e)$. 
Proof. We take $Y=G, y_{0}=e \in G(k)$, and $x_{0}=\pi\left(y_{0}\right)=\pi(e)$ in Lemma 3.3, then $\operatorname{Br}_{1, x_{0}}(X, Y)=\mathrm{Br}_{1, x_{0}}(X, G)$ and $\pi^{*} b\left(y_{0} . g\right)=\pi^{*} b(g)$.

Corollary 3.5. Let $k$ be a number field. Let $X:=H \backslash G$ be a homogeneous space of a connected linear $k$-group $G$, where $H \subset G$ is a connected linear $k$-subgroup. Let $\langle$,$\rangle denote the Manin pairing$

$$
\operatorname{Br}(X) \times X(\mathbf{A}) \rightarrow \mathbb{Q} / \mathbb{Z}
$$

Let $b \in \mathrm{Br}_{1, x_{0}}(X, G), x \in X(\mathbf{A}), g \in G(\mathbf{A})$. Then

$$
\langle b, x . g\rangle=\langle b, x\rangle+\left\langle\pi^{*}(b), g\right\rangle,
$$

where $\pi: G \rightarrow X$ is the quotient map and $x_{0}=\pi(e)$.

Corollary 3.6. Let $k, G, H$ and $X$ be as in Corollary 3.5. Let $\varphi: G^{\prime} \rightarrow G$ be a homomorphism of $k$-groups, where $G^{\prime}$ is a simply connected $k$-group. Let $b \in$ $\mathrm{Br}_{1, x_{0}}(X, G), x \in X(\mathbf{A}), g^{\prime} \in G^{\prime}(\mathbf{A})$. Then

$$
\left\langle b, x \cdot \varphi\left(g^{\prime}\right)\right\rangle=\langle b, x\rangle .
$$

Proof. By Corollary 3.5 we have

$$
\left\langle b, x \cdot \varphi\left(g^{\prime}\right)\right\rangle=\langle b, x\rangle+\left\langle\pi^{*}(b), \varphi\left(g^{\prime}\right)\right\rangle .
$$

By functoriality we have

$$
\left\langle\pi^{*}(b), \varphi\left(g^{\prime}\right)\right\rangle=\left\langle\varphi^{*} \pi^{*}(b), g^{\prime}\right\rangle .
$$

Since $b \in \operatorname{Br}_{1, x_{0}}(X, G)$, we have $\pi^{*} b \in \operatorname{Br}_{1, e}(G)$ and $\varphi^{*} \pi^{*} b \in \operatorname{Br}_{1, e}\left(G^{\prime}\right)=0$. Thus $\varphi^{*} \pi^{*} b=0$, hence $\left\langle\pi^{*}(b), \varphi\left(g^{\prime}\right)\right\rangle=0$, and the corollary follows.

\section{Some lemmas}

For an abelian group $A$ we write $A^{D}:=\operatorname{Hom}(A, \mathbb{Q} / \mathbb{Z})$.

Lemma 4.1. Let $P$ be a quasi-trivial $k$-torus over a number field $k$. Then the canonical map $\lambda: P(\mathbf{A}) \rightarrow \mathrm{Br}_{\mathrm{a}}(P)^{D}$ induced by the Manin pairing is surjective.

Proof. We have $\operatorname{Br}_{\mathrm{a}}(P)=H^{2}(k, \widehat{P})$, see [38], Lemma 6.9 (ii). By [38], (8.11.2), the map

$$
\lambda: P(\mathbf{A}) \rightarrow \operatorname{Br}_{\mathrm{a}}(P)^{D}=H^{2}(k, \widehat{P})^{D}
$$

is given by the canonical pairing

$$
P(\mathbf{A}) \times H^{2}(k, \widehat{P}) \rightarrow \mathbb{Q} / \mathbb{Z} .
$$


Consider the map $\mu$ from the Tate-Poitou exact sequence

$$
\left(P(\mathbf{A})_{\bullet}\right)^{\wedge} \stackrel{\mu}{\longrightarrow} H^{2}(k, \widehat{P})^{D} \rightarrow H^{1}(k, P),
$$

see [25], Theorem 5.6 or [14], Theorem 6.3. By $(P(\mathbf{A}) \bullet)^{\wedge}$ we mean the completion of $P(\mathbf{A})$. for the topology of open subgroups of finite index. Then the map $\mu$ is induced by $\lambda$. Since $P$ is a quasi-trivial torus, we have $H^{1}(k, P)=0$, and we see from (28) that the map $\mu$ is surjective. But by [22], Lemma $4, \operatorname{im} \mu=\operatorname{im} \lambda$. Thus $\lambda$ is surjective.

Lemma 4.2. Let $X$ be a right homogeneous space (not necessarily principal) of a connected $k$-group $G$ over a number field $k$. Let $N \subset G$ be a connected normal $k$-subgroup. Set $Y:=X / N$, and let $\pi: X \rightarrow Y$ be the canonical map. Then the induced map $X(\mathbf{A}) \rightarrow Y(\mathbf{A})$ is open.

Note that the geometric quotient $X / N$ exists in the category of $k$-varieties by [5], Lemma 3.1.

Proof. If $v$ is a nonarchimedean place of $k$, we denote by $\mathcal{O}_{v}$ the ring of integers of $k_{v}$, and by $\kappa_{v}$ the residue field of $\mathcal{O}_{v}$. For an $\mathcal{O}_{v}$-scheme $Z_{v}$ we set $\widetilde{Z}_{v}:=Z_{v} \times \mathcal{O}_{v} \kappa_{v}$.

Since the morphism $\pi$ is smooth, the map $X\left(k_{v}\right) \rightarrow Y\left(k_{v}\right)$ is open for any place $v$ of $k$.

Let $S$ be a finite set of places of $k$ containing all the archimedean places. Write $\mathcal{O}^{S}$ for the ring of elements of $k$ that are integral outside $S$. Taking $S$ sufficiently large, we can assume that $G$ and $N$ extend to smooth group schemes $\mathcal{G}$ and $\mathcal{N}$ over $\operatorname{Spec}\left(\mathcal{O}^{S}\right)$, and that $X$ and $Y$ extend to homogeneous spaces $\mathcal{X}$ of $\mathcal{E}$ and $\mathcal{Y}$ of $\mathcal{G} / \mathcal{N}$ over $\operatorname{Spec}\left(\mathcal{O}^{S}\right)$ such that $\mathcal{Y}=\mathcal{X} / \mathcal{N}$. In particular, the reduction $\tilde{N}_{v}:=\mathcal{N} \times_{\mathcal{O}^{S}} \kappa_{v}$ is connected for $v \notin S$.

Let $v \notin S$ and let $y_{v} \in \mathcal{Y}\left(\mathcal{O}_{v}\right)$. Set $X_{y_{v}}:=\mathcal{X} \times y \operatorname{Spec}\left(\mathcal{O}_{v}\right)$, the morphisms being given by $\pi$ and by $y_{v}: \operatorname{Spec}\left(\mathcal{O}_{v}\right) \rightarrow \mathcal{Y}$. It is an $\mathcal{O}_{v}$-scheme. Then its reduction $\widetilde{X_{y_{v}}}$ is a homogeneous space of the connected $\kappa_{v}$-group $\tilde{N}_{v}$ over the finite field $\kappa_{v}$. By Lang's theorem ([31], Theorem 2) $\widetilde{X_{y_{v}}}$ has a $\kappa_{v}$-point. By Hensel's lemma $X_{y_{v}}$ has an $\mathcal{O}_{v}$-point. This means that $y_{v} \in \pi\left(\mathcal{X}\left(\mathcal{O}_{v}\right)\right)$. Thus $\pi\left(\mathcal{X}\left(\mathcal{O}_{v}\right)\right)=\mathcal{Y}\left(\mathcal{O}_{v}\right)$ for all $v \notin S$. It follows that the map $X(\mathbf{A}) \rightarrow Y(\mathbf{A})$ is open.

4.3. Let $X=H \backslash G$ be a homogeneous space of a connected $k$-group $G$. Let $x_{1} \in X(k)$. Consider the map $\pi_{x_{1}}: G \rightarrow X, g \mapsto x_{1} . g$, it induces a homomorphism $\pi_{x_{1}}^{*}: \operatorname{Br}(X) \rightarrow \operatorname{Br}(G)$. Let $\operatorname{Br}_{1}(X, G)_{x_{1}}$ denote the subgroup of elements $b \in \operatorname{Br}(X)$ such that $\pi_{x_{1}}^{*}(b) \in \operatorname{Br}_{1}(G)$. The following lemma shows that $\operatorname{Br}_{1}(X, G)_{x_{1}}$ does not depend on $x_{1}$, so we may write $\operatorname{Br}_{1}(X, G)$ instead of $\operatorname{Br}_{1}(X, G)_{x_{1}}$. Note that $\mathrm{Br}_{1}(X, G)=\mathrm{Br}_{1, x_{1}}(X, G)+\operatorname{Br}(k)$.

Lemma 4.4. The subgroup $\operatorname{Br}_{1}(X, G)_{x_{1}} \subset \operatorname{Br}(X)$ does not depend on $x_{1}$. 
Proof. We have a commutative diagram

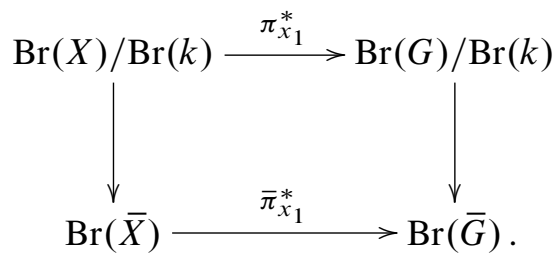

We see that it suffices to prove that the kernel of $\bar{\pi}_{x_{1}}^{*}$ does not depend on $x_{1}$.

Now if $x_{2} \in X(k)$ is another $k$-point, then $x_{2}=x_{1} . g$ for some $g \in G(\bar{k})$, hence

$$
\bar{\pi}_{x_{2}}\left(g^{\prime}\right)=x_{2} \cdot g^{\prime}=x_{1} \cdot g g^{\prime}=\bar{\pi}_{x_{1}}\left(g g^{\prime}\right)=\left(\bar{\pi}_{x_{1}} \circ l_{g}\right)\left(g^{\prime}\right),
$$

where $l_{g}$ denotes the left translation on $\bar{G}$ by $g$. Thus

$$
\bar{\pi}_{x_{2}}^{*}=l_{g}^{*} \circ \bar{\pi}_{x_{1}}^{*} .
$$

Since $l_{g}$ is an isomorphism of the underlying variety of $\bar{G}$, we see that $l_{g}^{*}: \operatorname{Br}(\bar{G}) \rightarrow$ $\operatorname{Br}(\bar{G})$ is an isomorphism, hence ker $\bar{\pi}_{x_{2}}^{*}=\operatorname{ker} \bar{\pi}_{x_{1}}^{*}$, which proves the lemma.

Lemma 4.5. Let $X$ be a right homogeneous space of a unipotent $k$-group $U$ over a field $k$ of characteristic 0 . Then $X(k)$ is non-empty and is one orbit of $U(k)$.

Proof. By [5], Lemma 3.2(i), $X(k) \neq \emptyset$. Let $x \in X(k), H:=\operatorname{Stab}(x)$, then $H$ is unipotent, hence $H^{1}(k, H)=1$, and therefore $X(k)=x \cdot U(k)$.

Corollary 4.6. Let $X$ be a right homogeneous space of a unipotent $\mathbb{R}$-group $U$. Then $X(\mathbb{R})$ is non-empty and connected.

Proof. Since $U(\mathbb{R})$ is connected and $X(\mathbb{R})=x \cdot U(\mathbb{R})$, we conclude that $X(\mathbb{R})$ is connected.

Lemma 4.7. Let $G$ be a unipotent $k$-group over a number field $k$. Let $X$ be a right homogeneous space of $G$. Let $S \subset \Omega$ be any non-empty finite set of places. Then $X(k)$ is non-empty and dense in $X\left(\mathbf{A}^{S}\right)$.

Proof. By [5], Lemma 3.2 (i), $X(k)$ is non-empty. Let $x_{0} \in X(k)$, and let $H \subset G$ denote the stabilizer of $x_{0}$ in $G$. We have $X=H \backslash G$.

Set $\mathrm{g}=\operatorname{Lie}(G)$. Since $\mathrm{g}$ is a vector space and $S \neq \emptyset$, by the classical strong approximation theorem $\mathfrak{g}$ is dense in $\mathfrak{g} \otimes_{k} \mathbf{A}^{S}$. Since $\operatorname{char}(k)=0$, we have the exponential map $\mathrm{g} \rightarrow G$, which is an isomorphism of $k$-varieties. We see that $G(k)$ is dense in $G\left(\mathbf{A}^{S}\right)$. It follows that $x_{0} G(k)$ is dense in $x_{0} G\left(\mathbf{A}^{S}\right)$. Since $H$ is unipotent, we have $H^{1}\left(k_{v}, H\right)=0$ for any $v \in \Omega$, and therefore $x_{0} G\left(k_{v}\right)=X\left(k_{v}\right)$ for any $v$. It follows that $x_{0} G\left(\mathbf{A}^{S}\right)=X\left(\mathbf{A}^{S}\right)$ (we use Lang's theorem and Hensel's lemma). Thus $x_{0} G(k)$ is dense in $X\left(\mathbf{A}^{S}\right)$, and $X(k)$ is dense in $X\left(\mathbf{A}^{S}\right)$. 


\section{Brauer group}

We are grateful to A. N. Skorobogatov, E. Shustin, and T. Ekedahl for helping us to prove Theorem 5.1 below.

Theorem 5.1. Let $X$ be a smooth irreducible algebraic variety over an algebraically closed field $k$ of characteristic 0 . Let $G$ be a connected algebraic group (not necessarily linear) defined over $k$, acting on $X$. Then $G(k)$ acts on $\operatorname{Br}(X)$ trivially.

Proof. We write $H^{i}$ for $H_{\text {ét }}^{i}$ (étale cohomology). The Kummer exact sequence

$$
1 \rightarrow \mu_{n} \rightarrow \mathbb{G}_{m} \stackrel{n}{\longrightarrow} \mathbb{G}_{m} \rightarrow 1
$$

of multiplication by $n$ gives rise to a surjective map

$$
H^{2}\left(X, \mu_{n}\right) \rightarrow \operatorname{Br}(X)_{n},
$$

where $\operatorname{Br}(X)_{n}$ denotes the group of elements of order dividing $n$ in $\operatorname{Br}(X)$. Since every element of $\operatorname{Br}(X)$ is torsion (because $\operatorname{Br}(X)$ embeds in $\operatorname{Br}(k(X))$, cf. [20], II, Corollary 1.8), it is enough to prove the following Theorem 5.2.

Theorem 5.2. Let $X$ be a smooth irreducible algebraic variety over an algebraically closed field $k$ (of any characteristic). Let $G$ be a connected algebraic group (not necessarily linear) defined over $k$, acting on $X$. Let $A$ be a finite abelian group of order invertible in $k$. Then $G(k)$ acts on $H_{\text {ét }}^{i}(X, A)$ trivially for all $i$.

Proof in characteristic 0 . By the Lefschetz principle, we may assume that $k=\mathbb{C}$. Let $g \in G(\mathbb{C})$. We must prove that $g$ acts trivially on the Betti cohomology $H_{B}^{i}(X, A)$. Since $G$ is connected, the group $G(\mathbb{C})$ is connected, hence we can connect $g$ with the unit element $e \in G(\mathbb{C})$ by a path. We see that the automorphism of $X$

$$
g_{*}: X \rightarrow X, \quad x \mapsto x \cdot g
$$

is homotopic to the identity automorphism

$$
e_{*}: X \rightarrow X, \quad x \mapsto x
$$

It follows that $g_{*}$ acts on $H_{B}^{i}(X, A)$ as $e_{*}$, i.e. trivially.

To prove Theorem 5.2 in any characteristic, we need two lemmas.

Lemma 5.3. Let $X, Y$ be smooth algebraic varieties over an algebraically closed field $k$ (of any characteristic). Let $A$ be a finite abelian group of order invertible in $k$. Consider the projection $p_{Y}: X \times Y \rightarrow Y$. Then the higher direct image $R^{i}\left(p_{Y}\right)_{*} A$ in the étale topology is the pullback of the abelian group $H^{i}(X, A)$ considered as a sheaf on $\operatorname{Spec}(k)$. 
Proof. Consider the commutative diagram

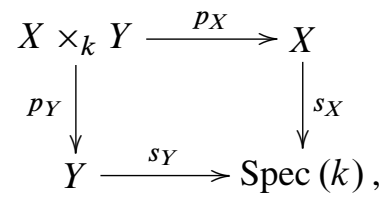

here $X \times_{k} Y$ is the fibre product of $X$ and $Y$ with respect to the structure morphisms $s_{X}$ and $s_{Y}$. Clearly $R^{i}\left(s_{X}\right)_{*} A$ is the constant sheaf on $\operatorname{Spec}(k)$ with stalk $H^{i}(X, A)$. By [13], Th. finitude, Theorem 1.9 (ii), the sheaf $R^{i}\left(p_{Y}\right)_{*} A$ on $Y$ is the pullback of the constant sheaf $R^{i}\left(s_{X}\right)_{*} A$ on $\operatorname{Spec}(k)$ along the morphism $s_{Y}: Y \rightarrow \operatorname{Spec}(k)$, which concludes the proof.

Let $y \in Y(k)$. It defines a canonical morphism $f_{y}: X \rightarrow X \times_{k} Y$ such that

$$
p_{X} \circ f_{y}=\mathrm{id}_{X} \text { and } p_{Y} \circ f_{y}=y \circ s_{X}: X \rightarrow Y .
$$

Lemma 5.4. Let $X, Y$ be two smooth algebraic varieties over an algebraically closed field $k$ (of any characteristic). Let $A$ be a finite abelian group of order invertible in $k$. For a closed point $y \in Y$ consider the map $f_{y}: X \rightarrow X \times Y$ defined above. If $Y$ is irreducible, then the map

$$
f_{y}^{*}: H^{i}(X \times Y, A) \rightarrow H^{i}(X, A)
$$

does not depend on $y$.

Proof. Let $\eta \in H^{i}(X \times Y, A)$. Then $\eta$ defines a global section $\lambda(\eta)$ of the sheaf $R^{i}\left(p_{Y}\right)_{*} A$ (via compatible local sections $\left.U \mapsto \eta\right|_{X \times U} \in H^{i}(X \times U, A)$ of the corresponding presheaf, for all étale open subsets $U \rightarrow Y$ ). By Lemma 5.3 the sheaf $R^{i}\left(p_{Y}\right)_{*} A$ is a constant sheaf with stalk $H^{i}(X, A)$. It is easy to see that

$$
f_{y}^{*}(\eta)=\lambda(\eta)(y) \in H^{i}(X, A) .
$$

Since $Y$ is irreducible, it is connected, hence the global section $\lambda(\eta)$ of the constant sheaf $R^{i}\left(p_{Y}\right)_{*} A$ on $Y$ is constant, and therefore $\lambda(\eta)(y)$ does not depend on $y$. Thus $f_{y}^{*}(\eta)$ does not depend on $y$.

Proof of Theorem 5.2 in any characteristic. Consider the map

$$
m: X \times G \rightarrow X, \quad(x, g) \mapsto x \cdot g
$$

(the action). Let $\xi \in H^{i}(X, A)$. Set $\eta=m^{*} \xi \in H^{i}(X \times G, A)$. For a $k$-point $g \in G(k)$ consider the map $f_{g}: X \rightarrow X \times G$ defined by $x \mapsto(x, g)$, as above. Since $x . g=m(x, g)=m\left(f_{g}(x)\right)$, we have

$$
g^{*} \xi=f_{g}^{*} m^{*} \xi=f_{g}^{*} \eta \in H^{i}(X, A) .
$$

By Lemma $5.4 f_{g}^{*} \eta$ does not depend on $g$. Thus $g^{*} \xi$ does not depend on $g$. This means that $G(k)$ acts on $H^{i}(X, A)$ trivially. 


\section{Homogeneous spaces of simply connected groups}

In the proof of the main theorem we shall need a result about strong approximation in homogeneous spaces of semisimple simply connected groups with connected stabilizers. If $X=H \backslash G$ is such a homogeneous space, since $G$ is semisimple and simply connected, the group $\operatorname{Br}(\bar{G})$ is trivial (see [18], corollary in the Introduction), hence $\operatorname{Br}_{1, x_{0}}(X, G)=\operatorname{Br}_{x_{0}}(X)$.

Theorem 6.1 (Colliot-Thélène and $\mathrm{Xu}$ ). Let $\mathrm{G}$ be a semisimple simply connected $k$-group over a number field $k$, and let $H \subset G$ be a connected subgroup. Set $X:=H \backslash G$. Let $S$ be a non-empty finite set of places of $k$ such that $G(k)$ is dense in $G\left(\mathbf{A}^{S}\right)$. Then the set of points $x \in X(\mathbf{A})$ such that $\langle b, x\rangle=0$ for all $b \in \mathrm{Br}_{1, x_{0}}(X, G)=\mathrm{Br}_{x_{0}}(X)$ coincides with the closure of the set $X(k) . G\left(k_{S}\right)$ in $X(\mathbf{A})$ for the adelic topology.

Proof. This is very close to a result of Colliot-Thélène and $\mathrm{Xu}$, see [11], Theorem 3.7 (b). Since their result is not stated in these terms in [11], we give here a proof of Theorem 6.1, following their argument.

We prove the nontrivial inclusion of the theorem. Let $x \in X(\mathbf{A})$ be orthogonal to $\mathrm{Br}_{x_{0}}(X)$. Then by [11], Theorem 3.3, there exists $x_{1} \in X(k)$ and $g \in G(\mathbf{A})$ such that $x=x_{1} . g$. Let $\mathcal{U}_{X} \subset X(\mathbf{A})$ be an open neighbourhood of $x$. Clearly there exists an open neighbourhood $U_{G} \subset G(\mathbf{A})$ of $g$ such that for any $g^{\prime} \in U_{G}$ we have $x_{1} . g^{\prime} \in U_{X}$. By assumption $G(k)$ is dense in $G\left(\mathbf{A}^{S}\right)$, hence $G(k) . G\left(k_{S}\right)$ is dense in $G(\mathbf{A})$. It follows that there exist $g_{0} \in G(k)$ and $g_{S} \in G\left(k_{S}\right)$ such that $g_{0} g_{S} \in U_{G}$, then $x_{1} . g_{0} . g_{S} \in \mathcal{U}_{X}$. Set $x_{2}=x_{1} . g_{0}$, then $x_{2} \in X(k)$ and $x_{2} . g_{S} \in \mathcal{U}_{X}$. Thus $x$ lies in the closure of the set $X(k) . G\left(k_{S}\right)$ in $X(\mathbf{A})$ for the adelic topology. This proves the nontrivial inclusion.

We prove the trivial inclusion. Let $x_{1} \in X(k), g_{S} \in G\left(k_{S}\right)$ and $b \in \operatorname{Br}_{1, x_{0}}(X, G)$. Clearly we have $\left\langle b, x_{1}\right\rangle=0$. By Corollary 3.6 we have $\left\langle b, x_{1} \cdot g_{S}\right\rangle=\left\langle b, x_{1}\right\rangle=0$. By Lemma 6.2 below we have $\langle b, x\rangle=0$ for any $x$ in the closure of the set $X(k) \cdot G\left(k_{S}\right)$.

Lemma 6.2. Let $X$ be a smooth geometrically integral variety over a number field $k$. Let $b \in \operatorname{Br}(X)$. Then the function

$$
X(\mathbf{A}) \rightarrow \mathbb{Q} / \mathbb{Z}: x \mapsto\langle b, x\rangle
$$

is locally constant in $x$ for the adelic topology in $X(\mathbf{A})$.

Proof. Arguing as in [38], Proof of Lemma 6.2, we can reduce our lemma to the local case. In other words, it is enough to prove that for any completion $k_{v}$ of $k$ the function

$$
\phi_{b}: X\left(k_{v}\right) \rightarrow \operatorname{Br}\left(k_{v}\right): x \mapsto b(x)
$$


is locally constant in $x$. This follows from non-published results from the thesis of Antoine Ducros, cf. [16], Part II, Propositions (0.31) and (0.33).

Since those results of Ducros are not published, we give another proof of this fact. We are grateful to J.-L. Colliot-Thélène and to the referee for this proof. Let $x \in X\left(k_{v}\right)$. The problem is local, so we can replace $X$ by an affine open subset containing $x$. From now on, $X$ is assumed to be affine over $k_{v}$.

To show that the map $\phi_{b}$ is locally constant around $x$, we may replace $b$ by $b-b(x) \in \operatorname{Br}(X)$. Now we have $b(x)=0$ and we want to prove that $\phi_{b}$ is zero in a topological neighbourhood of $x$.

Since $X$ is a smooth affine $k_{v}$-variety, by a result by Hoobler (see [28], Corollary 1) there exists a class $\eta \in H^{1}\left(X, \mathrm{PGL}_{n}\right)$ such that $b$ is the image of $\eta$ by the usual coboundary map. The class $\eta$ is represented by an $X$-torsor $f: Y \rightarrow X$ under $\mathrm{PGL}_{n}$.

For $x^{\prime} \in X\left(k_{v}\right)$ let $\eta\left(x^{\prime}\right) \in H^{1}\left(k_{v}, \mathrm{PGL}_{n}\right)$ denote the image of $\eta$ under the map $\left(x^{\prime}\right)^{*}: H^{1}\left(X, \mathrm{PGL}_{n}\right) \rightarrow H^{1}\left(k_{v}, \mathrm{PGL}_{n}\right)$. Consider the exact sequence

$$
1=H^{1}\left(k_{v}, \mathrm{GL}_{n}\right) \rightarrow H^{1}\left(k_{v}, \mathrm{PGL}_{n}\right) \stackrel{\Delta}{\longrightarrow} \operatorname{Br}\left(k_{v}\right) .
$$

It is clear that $b\left(x^{\prime}\right)=\Delta\left(\eta\left(x^{\prime}\right)\right)$. From the exact sequence we see that $b\left(x^{\prime}\right)=0$ if and only if $\eta\left(x^{\prime}\right)=1$. On the other hand, clearly $\eta\left(x^{\prime}\right)$ is the class of the $k_{v}$-torsor $f^{-1}\left(x^{\prime}\right)$ under PGL P $_{n}$. It follows that $b\left(x^{\prime}\right)=0$ if and only if $f^{-1}\left(x^{\prime}\right)$ contains a $k_{v}$-point, i.e. $x^{\prime}=f(y)$ for some $y \in Y\left(k_{v}\right)$. Hence the set of points $x^{\prime} \in X\left(k_{v}\right)$ such that $b\left(x^{\prime}\right)=0$ is exactly the subset $f\left(Y\left(k_{v}\right)\right)$ of $X\left(k_{v}\right)$. We now conclude by the implicit function theorem: since $f: Y \rightarrow X$ is a smooth morphism of $k_{v}$-schemes, the image $f\left(Y\left(k_{v}\right)\right)$ is an open subset of $X\left(k_{v}\right)$. Therefore, $\phi_{b}$ is zero on the open neighbourhood $f\left(Y\left(k_{v}\right)\right)$ of $x$, which concludes the proof.

\section{Proof of the main theorem}

Throughout this section we consider $X=H \backslash G$ satisfying the assumptions of Theorem 1.4. Let $x \in X(\mathbf{A})$ be an adelic point, we write $x=\left(x^{f}, x_{\infty}\right)$, where $x^{f} \in X\left(\mathbf{A}^{f}\right), x_{\infty} \in X\left(k_{\infty}\right)$. Let $S$ be a finite set of places of $k$ containing all archimedean places, and we set $S_{f}:=S \cap \Omega_{f}$. Let $U_{X}^{f} \subset X\left(\mathbf{A}^{f}\right)$ be an open neighbourhood of $x^{f}$. For $v \in \Omega_{\infty}$, we set $U_{X, v}$ to be the connected component of $x_{v}$ in $X\left(k_{v}\right)$. We set $U_{X, \infty}:=\prod_{v \in \Omega_{\infty}} U_{X, v}$, then $U_{X, \infty}$ is the connected component of $x_{\infty}$ in $X\left(k_{\infty}\right)$. We set

$$
u_{X}:=u_{X}^{f} \times u_{X, \infty} \subset X(\mathbf{A})
$$

and

$$
u_{X}^{\prime}:=u_{X} \cdot G^{\mathrm{scu}}\left(k_{S_{f}}\right)=u_{X} \cdot G^{\mathrm{scu}}\left(k_{S}\right)=\mathcal{U}_{X}^{\prime} \times \mathcal{U}_{X, \infty}^{\prime},
$$


where $u_{X}^{\prime}=u_{X}^{f} \cdot G^{\mathrm{scu}}\left(k_{S_{f}}\right)$ and $u_{X, \infty}^{\prime}=u_{X, \infty}=u_{X, \infty} \cdot G^{\mathrm{scu}}\left(k_{\infty}\right)$ (because $G^{\mathrm{scu}}\left(k_{\infty}\right)$ is a connected topological group, see [34], Theorem 5.2.3). Then $\mathcal{U}_{X}$ and $u_{X}^{\prime}$ are open neighbourhoods of $x$ in $X(\mathbf{A})$. We say that $U_{X}$ is the special neighbourhood of $x$ defined by $u_{X}^{f}$.

For the sake of the argument it will be convenient to introduce Property $\left(\mathrm{P}^{S}\right)$ of a pair $(X, G)$ :

For any point $x \in X(\mathbf{A})$ orthogonal to $\operatorname{Br}_{1}(X, G)$, and for any open neigh$\left(\mathrm{P}^{S}\right)$ bourhood $U_{X}^{f}$ of $x^{f}$, the set $X(k) . G^{\mathrm{scu}}\left(k_{S}\right) \cap U_{X}$ (or equivalently the set $X(k) \cap U_{X}^{\prime}$, or equivalently the set $\left.X(k) \cdot G^{\mathrm{scu}}\left(k_{S_{f}}\right) \cap U_{X}\right)$ is non-empty, where $U_{X}$ is the special neighbourhood of $x$ defined by $u_{X}^{f}$.

The nontrivial part of Theorem 1.4 precisely says that property $\left(\mathrm{P}^{S}\right)$ holds for any $X, G$ and $S$ as in the theorem.

We start proving Theorem 1.4. The structure of the proof is somewhat similar to that of Theorem A.1 of [7].

7.1. First reduction. We reduce Theorem 1.4 to the case $G^{\mathrm{u}}=1$. Let $X$ and $G$ be as in the theorem. We represent $G$ as an extension

$$
1 \rightarrow G^{\text {lin }} \rightarrow G \rightarrow G^{\text {abvar }} \rightarrow 1,
$$

where $G^{\text {lin }}$ is a connected linear algebraic $k$-group and $G^{\text {abvar }}$ is an abelian variety over $k$. We use the notation of 1.1. Set $G^{\prime}:=G / G^{\mathrm{u}}, Y:=X / G^{\mathrm{u}}$. We have a canonical epimorphism $\varphi: G \rightarrow G^{\prime}$ and a canonical smooth $\varphi$-equivariant morphism $\psi: X \rightarrow Y$. We have $G^{\prime \text { lin }}=G^{\text {lin }} / G^{\mathrm{u}}$, hence $G^{\prime \text { u }}=1$. We have $G^{\prime \text { abvar }}=G^{\text {abvar }}$, hence $\amalg\left(G^{\prime \text { abvar }}\right)$ is finite.

Assume that the pair $\left(Y, G^{\prime}\right)$ has Property $\left(\mathrm{P}^{S}\right)$. We prove that the pair $(X, G)$ has this property. Let $x \in X(\mathbf{A})$ be a point orthogonal to $\operatorname{Br}_{1}(X, G)$. Set $y:=$ $\psi(x) \in Y(\mathbf{A})$. By functoriality, $y$ is orthogonal to $\operatorname{Br}_{1}\left(Y, G^{\prime}\right)$. Let $u_{X}^{f}$ be as in $\left(\mathrm{P}^{S}\right)$, and let $U_{X}, \mathcal{U}_{X}^{\prime}$ be the special neighbourhoods of $x$ defined by $U_{X}^{f}$. Note that $u_{X}^{\prime}={U_{X}^{\prime}}^{f} \times U_{X, \infty}$, where $U_{X}^{\prime}{ }^{f}$ is an open subset of $X\left(\mathbf{A}^{f}\right)$. Indeed, $G^{\text {scu }}\left(k_{v}\right)$ is connected for all $v \in \Omega_{\infty}$ (see [34], Theorem 5.2.3).

Set $U_{Y}^{f}:=\psi\left(u_{X}^{f}\right) \subset Y\left(\mathbf{A}^{f}\right), \mathcal{U}_{Y}:=\psi\left(\mathcal{U}_{X}\right) \subset Y(\mathbf{A})$ and $\mathcal{U}_{Y}^{\prime}:=\psi\left(\mathcal{U}_{X}^{\prime}\right) \subset$ $Y(\mathbf{A})$. Since $G^{\mathrm{u}}$ is connected, by Lemma $4.2 U_{Y}^{f}$ is open in $Y\left(\mathbf{A}^{f}\right)$ and $U_{Y}$ and $U_{Y}^{\prime}$ are open in $Y(\mathbf{A})$. Set $\mathcal{U}_{Y, v}:=\psi\left(\mathcal{U}_{X, v}\right)$. By [7], Lemma A.2, for each $v \in \Omega_{\infty}$ the set $\mathcal{U}_{Y, v}$ is the connected component of $y_{v}$ in $Y\left(k_{v}\right)$. Set

$$
u_{Y, \infty}:=\prod_{v \in \Omega_{\infty}} u_{Y, v}
$$


We have $u_{Y}=u_{Y}^{f} \times u_{Y, \infty}$. We see that $u_{Y}$ is the special neighbourhood of $y$ defined by $\mathcal{U}_{Y}^{f}$. From the split exact sequence

$$
1 \rightarrow G^{\mathrm{u}} \rightarrow G^{\mathrm{scu}} \rightarrow G^{\prime \mathrm{sc}} \rightarrow 1,
$$

we see that the map $G^{\mathrm{scu}}\left(k_{S}\right) \rightarrow{G^{\prime s c}}^{\text {sc }}\left(k_{S}\right)$ is surjective. Note that $G^{\prime s c u}=G^{\prime s c}$. It follows that

$$
u_{Y}^{\prime}=u_{Y} \cdot G^{\mathrm{scu}}\left(k_{S}\right)=u_{Y} \cdot G^{\prime \mathrm{sc}}\left(k_{S}\right)=u_{Y} \cdot G^{\prime \mathrm{scu}}\left(k_{S}\right) .
$$

Since the pair $\left(Y, G^{\prime}\right)$ has Property $\left(\mathrm{P}^{S}\right)$, there exists a $k$-point $y_{0} \in Y(k) \cap U_{Y}^{\prime}$.

Let $X_{y_{0}}$ denote the fibre of $X$ over $y_{0}$. It is a homogeneous space of the unipotent group $G^{\mathrm{u}}$. By Lemma 4.7, $X_{y_{0}}(k) \neq \varnothing$ and $X_{y_{0}}$ has the strong approximation property away from $\Omega_{\infty}$ : the set $X_{y_{0}}(k)$ is dense in $X_{y_{0}}\left(\mathbf{A}^{f}\right)$. Consider the set $\mathcal{V}^{f}:=X_{y_{0}}\left(\mathbf{A}^{f}\right) \cap \mathcal{U}_{X}^{\prime}$. By Corollary 4.6, for any $v \in \Omega_{\infty}$ the set $X_{y_{0}}\left(k_{v}\right)$ is connected, and by Lemma $4.5, X_{y_{0}}\left(k_{v}\right)$ is one orbit under $G^{\mathrm{u}}\left(k_{v}\right)$. Set $\mathcal{V}:=$ $\mathcal{V}^{f} \times X_{y_{0}}\left(k_{\infty}\right)$.

Let $v \in \Omega_{\infty}$. We show that $X_{y_{0}}\left(k_{v}\right) \subset \mathcal{U}_{X, v}$. Since $y_{0} \in \mathcal{U}_{Y}^{\prime}=\psi\left(\mathcal{U}_{X}^{\prime}\right)$, there exists a point $x_{v} \in U_{X, v}$ such that $y_{0}=\psi\left(x_{v}\right)$. Clearly $x_{v} \in X_{y_{0}}\left(k_{v}\right)$. Since $X_{y_{0}}\left(k_{v}\right)$ is one orbit under $G^{\mathrm{u}}\left(k_{v}\right)$, we see that $X_{y_{0}}\left(k_{v}\right)=x_{v} \cdot G^{\mathrm{u}}\left(k_{v}\right) \subset U_{X, v}$, because $G^{\mathrm{u}}\left(k_{v}\right)$ is a connected group. Thus $X_{y_{0}}\left(k_{\infty}\right) \subset U_{X, \infty}$ and $\mathcal{V} \subset \mathcal{U}_{X}^{\prime f} \times$ $u_{X, \infty}=U_{X}^{\prime}$, hence $\mathcal{V} \subset X_{y_{0}}(\mathbf{A}) \cap U_{X}^{\prime}$.

Since $y_{0} \in \psi\left(\mathcal{U}_{X}^{\prime}\right)$, the set $\mathcal{V}$ is non-empty. Since by Lemma $4.7 X_{y_{0}}(k)$ is dense in $X_{y_{0}}\left(\mathbf{A}^{f}\right)$, there is a point $x_{0} \in X_{y_{0}}(k) \cap \mathcal{V}$. Clearly $x_{0} \in X(k) \cap \mathcal{U}_{X}^{\prime}$. Thus the pair $(X, G)$ has Property $\left(\mathrm{P}^{S}\right)$. We see that in the proof of Theorem 1.4 we may assume that $G^{\mathrm{u}}=1$.

7.2. Second reduction. By [7], Proposition 3.1 we may regard $X$ as a homogeneous space of another connected group $G^{\prime}$ such that $G^{\prime \text { u }}=\{1\}, G^{\prime s s}$ is semisimple simply connected, and the stabilizers of the geometric points of $X$ in $G^{\prime}$ are linear and connected. We have $G^{\prime s c}=G^{\mathrm{sc}}$, hence $G^{\prime \mathrm{scu}}=G^{\mathrm{scu}}$ (because $G^{\mathrm{scu}}=G^{\mathrm{sc}}$ and $G^{\prime s c u}=G^{\prime s c}$ ). It follows from the construction in the proof of Proposition 3.1 of [7] that there is a surjective homomorphism $G^{\text {abvar }} \rightarrow G^{\text {abvar }}$. Since by assumption $\amalg\left(G^{\text {abvar }}\right)$ is finite, we obtain from [7], Lemma A.3 that $\amalg\left(G^{\text {abvar }}\right)$ is finite.

Let us prove that if a point $x \in X(\mathbf{A})$ is orthogonal to $\operatorname{Br}_{1}(X, G)$, then it is orthogonal to $\operatorname{Br}_{1}\left(X, G^{\prime}\right)$. More precisely, we prove that $\operatorname{Br}_{1}\left(X, G^{\prime}\right)$ is a subgroup of $\operatorname{Br}_{1}(X, G)$.

By construction (see [7], proof of Proposition 3.1), there is an exact sequence of connected algebraic groups

$$
1 \rightarrow S \rightarrow G^{\prime} \stackrel{q}{\rightarrow} G_{1} \rightarrow 1
$$


where $G_{1}$ is the quotient of $G$ by the central subgroup $Z(G) \cap H$ and $S$ is a $k$-torus. Consider the following natural commutative diagram

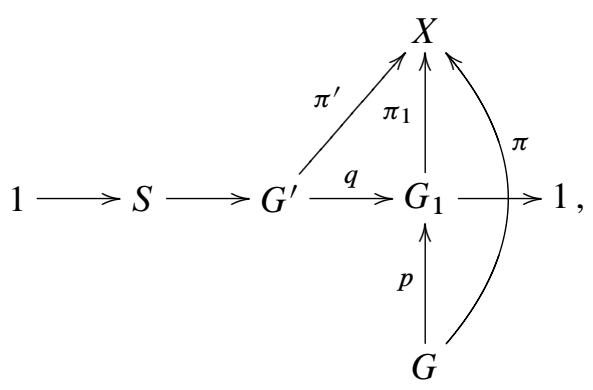

where the maps $\pi, \pi^{\prime}$ and $\pi_{1}$ are the natural quotient maps. From this diagram, we deduce the following one, where the second line is exact (see the top row of diagram (11)):

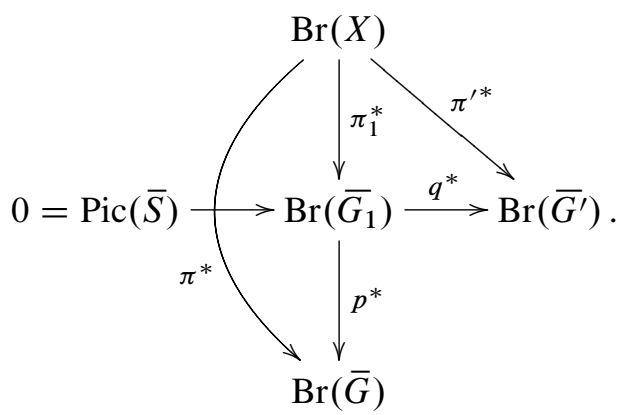

Therefore, the injectivity of the map $q^{*}: \operatorname{Br}\left(\bar{G}_{1}\right) \rightarrow \operatorname{Br}\left(\bar{G}^{\prime}\right)$ implies that the natural inclusion $\operatorname{Br}_{1, x_{0}}\left(X, G_{1}\right) \subset \operatorname{Br}_{1, x_{0}}\left(X, G^{\prime}\right)$ is an equality. And by functoriality $\mathrm{Br}_{1, x_{0}}\left(X, G_{1}\right)$ is a subgroup of $\mathrm{Br}_{1, x_{0}}(X, G)$.

Thus $\operatorname{Br}_{1, x_{0}}\left(X, G^{\prime}\right)=\operatorname{Br}_{1, x_{0}}\left(X, G_{1}\right)$ is a subgroup of $\operatorname{Br}_{1, x_{0}}(X, G)$. It follows that if a point $x \in X(\mathbf{A})$ is orthogonal to $\operatorname{Br}_{1}(X, G)$, then it is orthogonal to $\operatorname{Br}_{1}\left(X, G^{\prime}\right)$.

Thus if Theorem 1.4 holds for the pair $\left(X, G^{\prime}\right)$, then it holds for $(X, G)$. We see that we may assume in the proof of Theorem 1.4 that $G^{\text {lin }}$ is reductive, $G^{\mathrm{ss}}$ is simply connected, and the stabilizers of the geometric points of $X$ in $G$ are linear and connected.

Now in order to prove Theorem 1.4 it is enough to prove the following Theorem 7.3.

Theorem 7.3. Let $k$ be a number field, $G$ a connected $k$-group, and $X:=H \backslash G a$ homogeneous space of $G$ with connected stabilizer $H$. Assume: 
(i) $G^{\mathrm{u}}=\{1\}$,

(ii) $H \subset G^{\text {lin }}$, i.e. $H$ is linear,

(iii) $G^{\mathrm{ss}}$ is simply connected,

(iv) $\amalg\left(G^{\text {abvar }}\right)$ is finite.

Let $S \supset \Omega_{\infty}$ be a finite set of places of $k$ containing all archimedean places. We assume that $G^{\mathrm{sc}}(k)$ is dense in $G^{\mathrm{sc}}\left(\mathrm{A}^{S}\right)$. Then the pair $(X, G)$ has Property $\left(\mathrm{P}^{S}\right)$.

The homogeneous space $X$ defines a natural homomorphism $H^{\text {tor }} \rightarrow G^{\text {sab }}$. We first prove a crucial special case of Theorem 7.3.

Proposition 7.4. With the hypotheses of Theorem 7.3, assume that $H^{\text {tor }}$ injects into $G^{\mathrm{sab}}\left(\right.$ i.e. $\left.H \cap G^{\mathrm{ss}}=H^{\mathrm{ssu}}\right)$, and that the homomorphism $\mathrm{Br}_{1, e}\left(G^{\mathrm{sab}}\right) \rightarrow \mathrm{Br}_{1, e}\left(H^{\text {tor }}\right)$ is surjective. Then the pair $(X, G)$ has Property $\left(\mathrm{P}^{S}\right)$.

Construction 7.5. Set $Y:=X / G^{\text {ss }}$. Then $Y$ is a homogeneous space of the semiabelian variety $G^{\text {sab }}$, hence it is a torsor of some semiabelian variety $G^{\prime}$. We have $G^{\prime \text { abvar }}=G^{\text {abvar }}$, hence $\amalg\left(G^{\text {abvar }}\right)$ is finite. We have a canonical smooth morphism $\psi: X \rightarrow Y$.

To prove Proposition 7.4 we need a number of lemmas and propositions.

The following proposition is crucial for our proof of Proposition 7.4 by dévissage.

Proposition 7.6. Let $G, X$ be as in Proposition 7.4. Let $Y, \psi: X \rightarrow Y$ be as in Construction 7.5. Let $x_{0} \in X(k), y_{0}:=\psi\left(x_{0}\right), X_{y_{0}}:=\psi^{-1}\left(y_{0}\right)=x_{0} . G^{\mathrm{ss}}$. Then the natural pullback homomorphism

$$
i^{*}: \mathrm{Br}_{1, x_{0}}(X, G) \rightarrow \mathrm{Br}_{x_{0}}\left(X_{y_{0}}\right)
$$

is surjective.

Note that $\mathrm{Br}_{1, x_{0}}\left(X_{y_{0}}, G^{\mathrm{ss}}\right)=\mathrm{Br}_{x_{0}}\left(X_{y_{0}}\right)$ because $\operatorname{Br}\left(\bar{G}^{\mathrm{ss}}\right)=0$.

Construction 7.7. Consider the map $\pi_{x_{0}}: G \rightarrow X, g \mapsto x_{0} . g$. This map identifies $X$ (resp. $X_{y_{0}}$ ) with a quotient of $G\left(\right.$ resp. $G^{\text {ss }}$ ) by a connected subgroup $H^{\prime}$ (resp. $\left.H^{\prime \text { ssu }}\right)$, so we have

$$
X=H^{\prime} \backslash G
$$

and

$$
X_{y_{0}}=H^{\prime \text { ssu }} \backslash G^{\mathrm{ss}} .
$$


We define a $k$-variety $Z$ by

$$
Z:=H^{\prime s s u} \backslash G
$$

and denote by $z_{0} \in Z(k)$ the image of $e \in G(k)$. We have a commutative diagram of $k$-varieties:

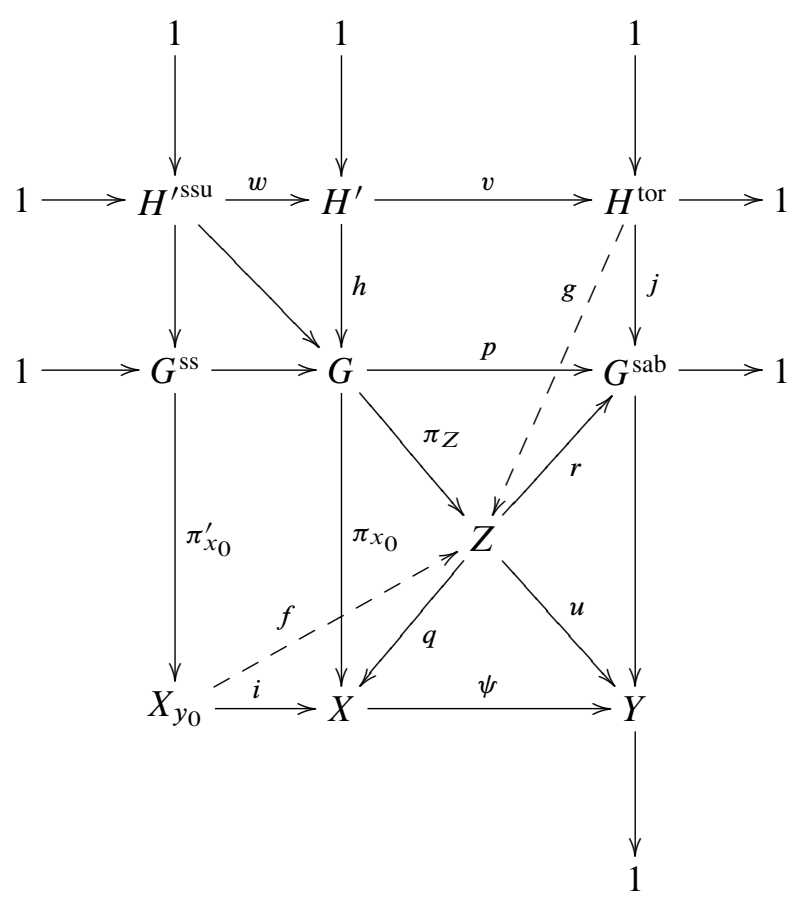

where the first two rows and the last column are exact sequences of connected algebraic groups, and the other maps are the natural maps between the different homogeneous spaces.

The following two lemmas are versions of exact sequence (9) of Theorem 2.8.

Lemma 7.8. The following sequence is exact:

$$
\mathrm{Br}_{1, e}\left(G^{\mathrm{sab}}\right) \stackrel{r^{*}}{\rightarrow} \mathrm{Br}_{1, z_{0}}(Z, G) \stackrel{f^{*}}{\rightarrow} \mathrm{Br}_{x_{0}}\left(X_{y_{0}}\right) \rightarrow 0
$$

Proof. We use the functoriality of exact sequence (9) of Theorem 2.8 to get the following commutative diagram with exact columns. Here the second column is the exact sequence (9) for $Z=H^{\prime \text { ssu }} \backslash G$ and the third column is exact sequence (9) 
applied to $X_{y_{0}}=H^{\prime \text { ssu }} \backslash G^{\text {ss }}$.

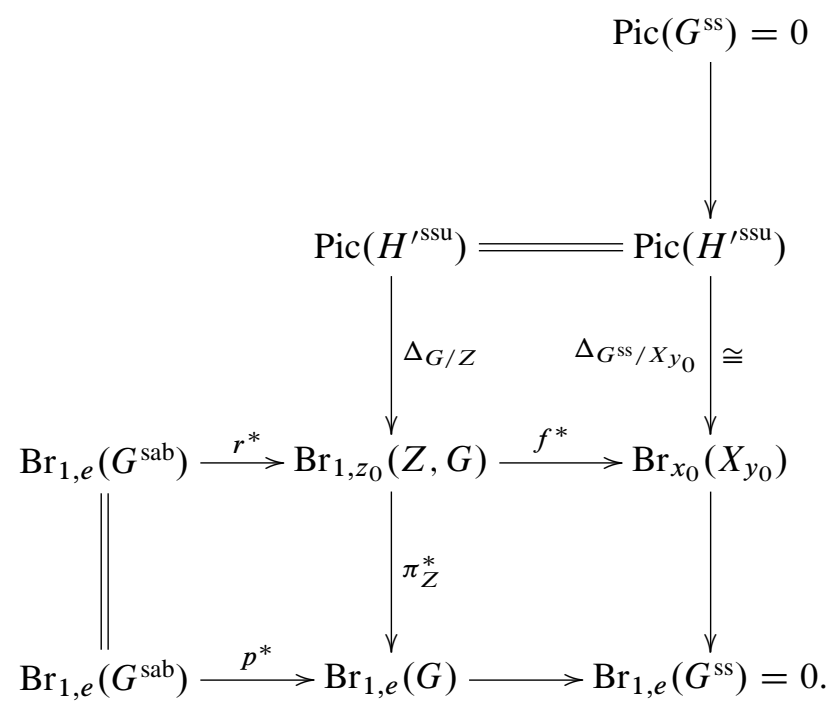

We have $\operatorname{Pic}\left(G^{\text {ss }}\right)=0$ and $\operatorname{Br}_{1, e}\left(G^{\text {ss }}\right)=0$ by [38], Lemma 6.9 (iv), because $G^{\text {ss }}$ is simply connected. From the bottom row of diagram (11) of Corollary 2.12 we get an exact sequence

$$
\mathrm{Br}_{1, e}\left(G^{\mathrm{sab}}\right) \stackrel{p^{*}}{\rightarrow} \mathrm{Br}_{1, e}(G) \stackrel{l^{*}}{\rightarrow} \mathrm{Br}_{1, e}\left(G^{\mathrm{ss}}\right),
$$

where $l: G^{\mathrm{ss}} \rightarrow G$ is the canonical embedding. $\operatorname{But}^{\operatorname{Br}_{1, e}}\left(G^{\mathrm{ss}}\right)=0$, therefore the homomorphism $p^{*}: \mathrm{Br}_{1, e}\left(G^{\mathrm{sab}}\right) \rightarrow \mathrm{Br}_{1, e}(G)$ is surjective. The composition $r \circ f$ being the trivial morphism, the second row of the diagram is a complex. A diagram chase in diagram (29) proves the exactness of the sequence of the lemma.

Lemma 7.9. The sequence

$$
\mathrm{Br}_{1, x_{0}}(X, G) \stackrel{q^{*}}{\rightarrow} \mathrm{Br}_{1, z_{0}}(Z, G) \stackrel{g^{*}}{\rightarrow} \mathrm{Br}_{1, e}\left(H^{\text {tor }}\right)
$$

is exact.

Proof. We consider the following diagram, in which the middle column and the last row are the exact sequences coming from exact sequence (9) of Theorem 2.8, and the last column is the exact sequence coming from the exact bottom row of diagram (11) 
of Corollary 2.12:

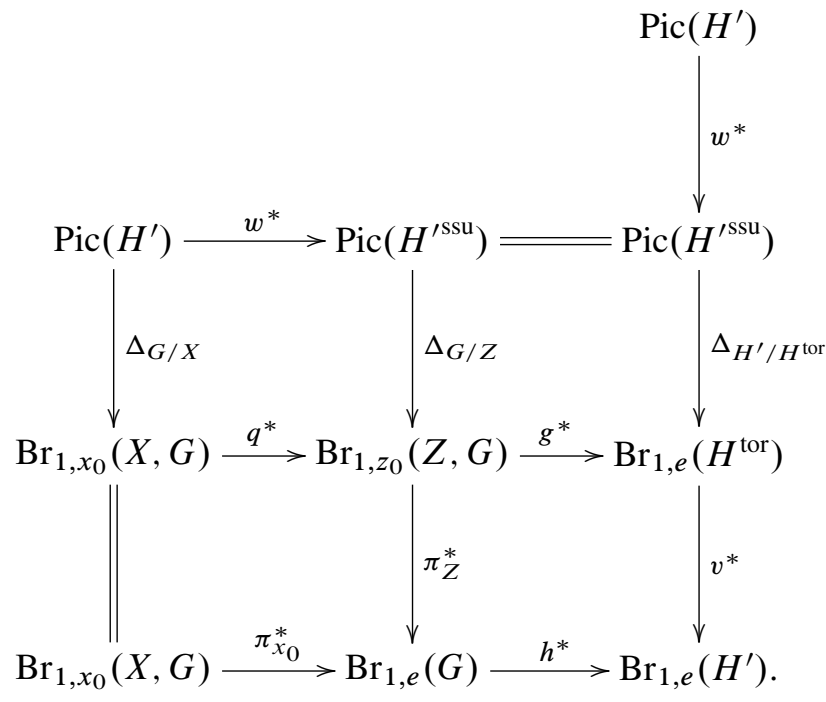

We prove that the diagram is commutative. In this diagram the two first columns define a commutative diagram by functoriality, and the second row is a complex. By construction we have $g \circ v=\pi_{Z} \circ h$, hence in the diagram we have $v^{*} \circ g^{*}=h^{*} \circ \pi_{Z}^{*}$. Let us prove the commutativity of the square in the top right-hand corner, i.e. let us prove that $g^{*} \circ \Delta_{G / Z}=\Delta_{H^{\prime} / H^{\text {tor }}}$. We observe that the following diagram of torsors under $H^{\prime \text { ssu }}$ is cartesian:

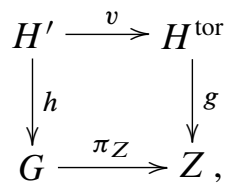

i.e. $\pi_{Z}^{-1}\left(H^{\text {tor }}\right)=H^{\prime}$. In other words, the $H^{\text {tor }}$-torsor $H^{\prime}$ is the pullback of the $Z$-torsor $G$ by the morphism $H^{\text {tor }} \stackrel{g}{\rightarrow} Z$. Therefore, if

$$
1 \rightarrow \mathbb{G}_{m} \rightarrow H_{1} \rightarrow H^{\prime \text { ssu }} \rightarrow 1
$$

is a central extension representing an element $p \in \operatorname{Pic}\left(H^{\prime s s u}\right)$ via the isomorphism $\operatorname{Ext}_{k}^{c}\left(H^{\prime s s u}, \mathbb{G}_{m}\right) \cong \operatorname{Pic}\left(H^{\prime s s u}\right)$, we get a commutative diagram:

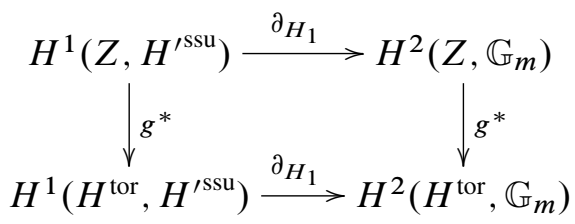


such that $g^{*}[G]=\left[H^{\prime}\right]$ in $H^{1}\left(H^{\text {tor }}, H^{\prime \text { ssu }}\right)$. We deduce from this diagram that $g^{*}\left(\partial_{H_{1}}([G])\right)=\partial_{H_{1}}\left(\left[H^{\prime}\right]\right)$ in $H^{2}\left(H^{\text {tor }}, \mathbb{G}_{m}\right)$, i.e. that $\Delta_{H^{\prime} / H^{\text {tor }}}(p)=g^{*}\left(\Delta_{G / Z}(p)\right)$ in $\operatorname{Br}\left(H^{\text {tor }}\right)$. Therefore the top right-hand square in diagram (30) is commutative.

Returning to diagram (30), we see that its commutativity and the exactness of the last two columns and of the last row imply, via an easy diagram chase, that the second row of (30) is also exact, hence the sequence of the lemma is exact.

For an alternative proof of Lemma 7.9 we need the following generalization of Proposition 2.8:

Proposition 7.10. Let $k$ be a field of characteristic zero, and

$$
1 \rightarrow H_{1} \rightarrow G \rightarrow H_{2} \rightarrow 1
$$

be an exact sequence of connected linear algebraic groups over $k$. Let $\pi: Z \rightarrow X$ and $\pi^{\prime}: Y \rightarrow Z$ be two morphisms of algebraic varieties such that the composite $Y \rightarrow X$ is an $X$-torsor under $G$, such that the restriction to $H_{1}$ of the action of $G$ on $Y$ defines the structure of a $Z$-torsor under $H_{1}$ on $Y$, and such that $Z \rightarrow X$ is a torsor under $\mathrm{H}_{2}$ via the induced action. Then we have a natural exact sequence

$$
\operatorname{Pic}(Z) \stackrel{\varphi_{1}}{\longrightarrow} \operatorname{Pic}\left(H_{2}\right) \stackrel{\Delta_{Z / X}}{\longrightarrow} \operatorname{Br}_{1}(X, Y) \stackrel{\pi^{*}}{\longrightarrow} \operatorname{Br}_{1}(Z, Y) \stackrel{\varphi_{2}^{\prime}}{\longrightarrow} \operatorname{Br}_{1, e}\left(H_{2}\right) .
$$

If in addition $z \in Z(k)$, we have an exact sequence

$$
\operatorname{Pic}(Z) \stackrel{i_{z}^{*}}{\rightarrow} \operatorname{Pic}\left(H_{2}\right) \stackrel{\Delta_{Z / X}}{\longrightarrow} \operatorname{Br}_{1, x}(X, Y) \stackrel{\pi^{*}}{\longrightarrow} \operatorname{Br}_{1, z}(Z, Y) \stackrel{i_{z}^{*}}{\rightarrow} \operatorname{Br}_{1, e}\left(H_{2}\right),
$$

where $x \in X(k)$ is the image of $z$.

Proof. As in the end of the proof of Theorem 2.8, we construct the exact sequence (31) from the top row of diagram (8) applied to the torsor $Z \rightarrow X$ :

$$
\operatorname{Pic}(Z) \stackrel{\varphi_{1}}{\longrightarrow} \operatorname{Pic}\left(H_{2}\right) \stackrel{\Delta_{Z / X}}{\longrightarrow} \operatorname{Br}(X) \stackrel{\pi^{*}}{\longrightarrow} \operatorname{Br}(Z) \stackrel{m^{*}-p_{Z}^{*}}{\longrightarrow} \operatorname{Br}\left(H_{2} \times Z\right) .
$$

Define a map $\varphi_{2}^{\prime}: \operatorname{Br}(Z, Y) \rightarrow \operatorname{Br}_{1, e}\left(H_{2}\right)$ to be the composition

$$
\begin{aligned}
\operatorname{Br}_{1}(Z, Y) \stackrel{m^{*}}{\longrightarrow} \operatorname{Br}_{1}\left(Z \times H_{2}, Y \times G\right) \stackrel{p_{Z}^{*}+p_{H_{2}}^{*}}{\longleftarrow} \operatorname{Br}_{1, e}\left(H_{2}, G\right) \stackrel{\oplus \operatorname{Br}_{1}(Z, Y)}{\stackrel{\pi_{H_{2}}}{\longrightarrow} \operatorname{Br}_{1, e}\left(H_{2}, G\right)}
\end{aligned}
$$

where the morphism $p_{Z}^{*}+p_{H_{2}}^{*}$ is an isomorphism by Lemma 3.1, and $\pi_{H_{2}}$ is the projection onto the first factor. Note that $\mathrm{Br}_{1, e}\left(H_{2}, G\right)=\mathrm{Br}_{1, e}\left(H_{2}\right)$ as a consequence of the injectivity of the homomorphism $\operatorname{Br}\left(\bar{H}_{2}\right) \rightarrow \operatorname{Br}(\bar{G})$, which comes from the exactness of the top row of diagram (11) of Corollary 2.12 and the fact that $\operatorname{Pic}(\bar{G}) \rightarrow$ $\operatorname{Pic}\left(\bar{H}_{1}\right)$ is onto (see [38], Remark 6.11.3). 
Consider the diagram

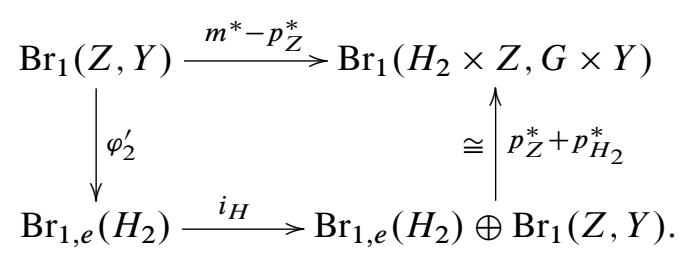

This diagram is commutative. Since $i_{H}$ is a canonical embedding, we see that $\operatorname{ker} \varphi_{2}^{\prime}=\operatorname{ker}\left(m^{*}-p_{Z}^{*}\right)$.

We prove that sequence (31) is exact. We use the exactness of (33). We know from Theorem 2.8 that the map $\Delta_{Z / X}: \operatorname{Pic}\left(H_{2}\right) \rightarrow \operatorname{Br}(X)$ lands in $\operatorname{Br}_{1}(X, Z)$. Since $\operatorname{Br}_{1}(X, Y) \supset \operatorname{Br}_{1}(X, Z)$, the map $\Delta_{Z / X}: \operatorname{Pic}\left(H_{2}\right) \rightarrow \operatorname{Br}_{1}(X, Y)$ is defined. We obtain a commutative diagram with an exact long horizontal line and exact vertical lines:

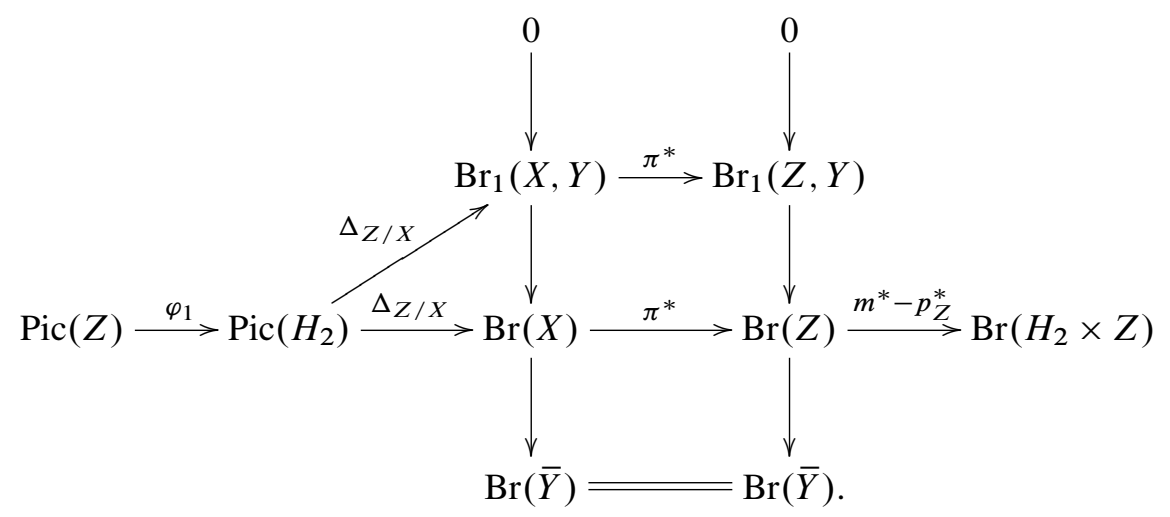

Now we see immediately that sequence (31) is exact at $\operatorname{Pic}\left(H_{2}\right)$ and $\operatorname{Br}_{1}(X, Y)$. Since $\operatorname{ker}\left(m^{*}-p_{Z}^{*}\right)=\operatorname{ker} \varphi_{2}^{\prime}$ in diagram (34), it follows from the exactness of (33) at $\operatorname{Br}(Z)$ that sequence (31) is exact at $\operatorname{Br}_{1}(Z, Y)$. Thus (31) is exact, which completes the proof.

7.11. Alternative proof of Lemma 7.9. We consider the exact sequence of linear algebraic groups

$$
1 \rightarrow H^{\text {ssu }} \rightarrow H^{\prime} \rightarrow H^{\text {tor }} \rightarrow 1
$$

and the torsors $\pi_{Z}: G \stackrel{H^{\prime \text { suu }}}{\longrightarrow} Z$ and $q: Z \stackrel{H^{\text {tor }}}{\longrightarrow} X$. The composition $\pi_{x_{0}}: G \rightarrow X$ is naturally a torsor under $H^{\prime}$. Therefore, we can apply Proposition 7.10 to get the exact sequence

$$
\operatorname{Pic}(Z) \stackrel{g^{*}}{\rightarrow} \operatorname{Pic}\left(H^{\text {tor }}\right) \stackrel{\Delta_{Z / X}}{\longrightarrow} \operatorname{Br}_{1, x_{0}}(X, G) \stackrel{q^{*}}{\rightarrow} \operatorname{Br}_{1, z_{0}}(Z, G) \stackrel{g^{*}}{\rightarrow} \operatorname{Br}_{1, e}\left(H^{\text {tor }}\right),
$$

which concludes the proof. 
7.12. Proof of Proposition 7.6. We have a commutative diagram

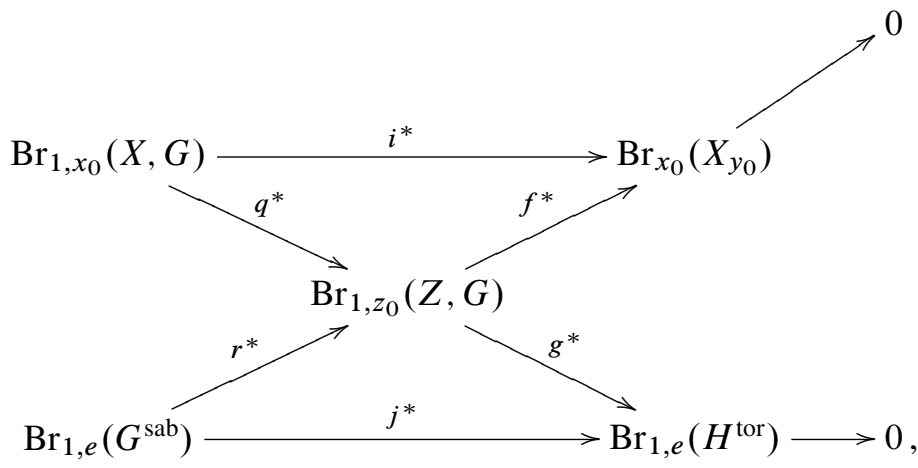

where the last row is exact by assumption, and the two slanted sequences are also exact by Lemmas 7.8 and 7.9. A diagram chase shows that the homomorphism

$$
\mathrm{Br}_{1, x_{0}}(X, G) \stackrel{i^{*}}{\rightarrow} \mathrm{Br}_{x_{0}}\left(X_{y_{0}}\right)
$$

is surjective, which completes the proof of Proposition 7.6.

For the proof of Proposition 7.4 we need three lemmas.

Lemma 7.13. Let $G, X$ be as in Proposition 7.4 and $Y:=X / G^{\mathrm{ss}}$. Let $\psi: X \rightarrow Y$ be the canonical map. Let $x_{1}, x_{2} \in X(k), y_{i}:=\psi\left(x_{i}\right), X_{i}:=X_{y_{i}}(i=1,2)$. Let $r_{i}: \operatorname{Br}_{1}(X, G) / \operatorname{Br}(k) \rightarrow \operatorname{Br}\left(X_{i}\right) / \operatorname{Br}(k)$ be the restriction homomorphisms. Then there exists a canonical isomorphism $\lambda_{1,2}: \operatorname{Br}\left(X_{1}\right) / \operatorname{Br}(k) \stackrel{\sim}{\longrightarrow} \operatorname{Br}\left(X_{2}\right) / \operatorname{Br}(k)$ such that the following diagram commutes:

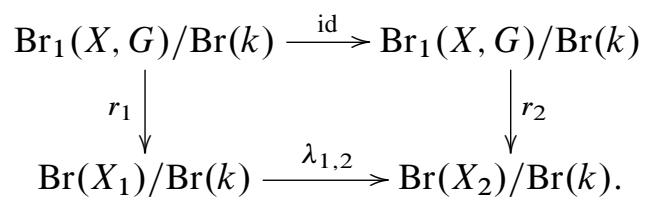

Proof. Choose $g \in G(\bar{k})$ such that $x_{1}=x_{2} \cdot g$. Then we have $\psi\left(x_{1}\right)=\psi\left(x_{2}\right) \cdot g$, hence $\psi^{-1}\left(\psi\left(x_{1}\right)\right)=\psi^{-1}\left(\psi\left(x_{2}\right)\right) . g$, thus $\bar{X}_{1}=\bar{X}_{2} . g$. We obtain commutative diagrams

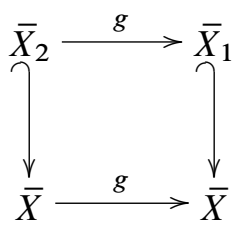

and

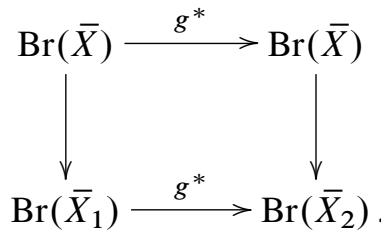


By Theorem $5.1 g^{*}: \operatorname{Br}(\bar{X}) \rightarrow \operatorname{Br}(\bar{X})$ is the identity map. Therefore, the following diagram

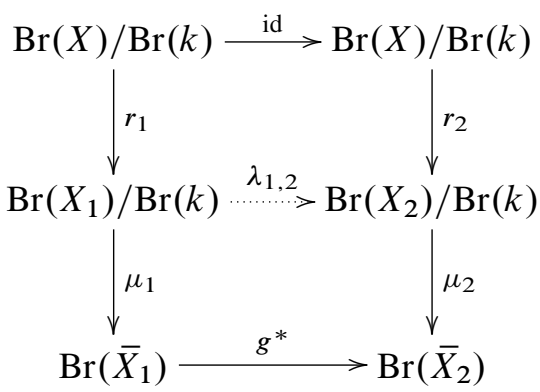

is commutative.

Here $r_{1}$ and $r_{2}$ are surjective by Proposition 7.6, while $\mu_{1}$ and $\mu_{2}$ are injective by Lemma 7.14 below. Clearly we can define the dotted arrow (in a unique way) such that the diagram with this new arrow will be also commutative. The top square of this new diagram is the desired diagram (36).

Lemma 7.14. Let $X:=H \backslash G$, where $G$ is a simply connected semisimple $k$-group over a field $k$ of characteristic 0 , and $H \subset G$ is a connected $k$-subgroup such that $H^{\text {tor }}=1$. Then $\operatorname{Br}(X) / \operatorname{Br}(k)$ is finite and the canonical homomorphism

$$
\operatorname{Br}(X) / \operatorname{Br}(k) \rightarrow \operatorname{Br}(\bar{X})
$$

is injective.

Proof. Let $x_{0}$ denote the image of $e \in G(k)$ in $X(k)$. We have a canonical isomorphism $\operatorname{Br}(X) / \operatorname{Br}(k) \cong \operatorname{Br}_{x_{0}}(X)$.

By Theorem 2.8 we have a canonical exact sequence

$$
\operatorname{Pic}(G) \rightarrow \operatorname{Pic}(H) \rightarrow \operatorname{Br}_{1, x_{0}}(X, G) \rightarrow \mathrm{Br}_{1, e}(G),
$$

where by [38], Lemma 6.9 (iv), we have $\operatorname{Pic}(G)=0$ and $\operatorname{Br}_{1, e}(G)=0$. Moreover, by [18] we have $\operatorname{Br}(\bar{G})=0$, hence $\operatorname{Br}_{1, x_{0}}(X, G)=\operatorname{Br}_{x_{0}}(X)$. We obtain a canonical isomorphism $\operatorname{Br}_{x_{0}}(X) \cong \operatorname{Pic}(H)$, functorial in $k$.

We have $H=H^{\text {ssu }}$, hence $\operatorname{Pic}(H) \cong \operatorname{Pic}\left(H^{\text {ss }}\right)$. In the commutative diagram

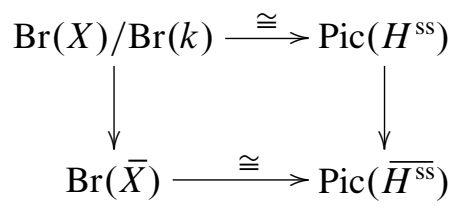

the right vertical arrow is clearly injective, hence so is the left one. 
Lemma 7.15. Let $X:=H \backslash G$, where $G$ is a connected $k$-group over a number field $k$, and $H \subset G$ is a connected linear $k$-subgroup. Let $M \subset \operatorname{Br}_{1}(X, G) / \operatorname{Br}(k)$ be a finite subset. Let $y:=\left(y_{f}, y_{\infty}\right) \in X\left(\mathbf{A}^{f}\right) \times X\left(k_{\infty}\right)=X(\mathbf{A})$ be a point orthogonal to $M$ with respect to the Manin pairing. Let $U_{\infty}$ denote the connected component of $y_{\infty}$ in $X\left(k_{\infty}\right)$. Then there exists an open neighbourhood $u^{f} \subset X\left(\mathbf{A}^{f}\right)$ of $y_{f}$ such that $\mathcal{U}^{f} \times \mathcal{U}_{\infty}$ is orthogonal to $M$.

Proof. It is an immediate consequence of Lemma 6.2, using the finiteness of $M$.

7.16. Recall that $X=H \backslash G$. Let $x_{0}$ be the image of $e \in G(k)$ in $X(k)$. Set $X_{0}:=x_{0} \cdot G^{\mathrm{ss}}$. By assumption $H \cap G^{\mathrm{ss}}=H^{\mathrm{ssu}}$. By Lemma $7.14 \operatorname{Br}\left(X_{0}\right) / \operatorname{Br}(k)$ is a finite group. By Proposition 7.6 the map $\operatorname{Br}_{1}(X, G) / \operatorname{Br}(k) \rightarrow \operatorname{Br}\left(X_{0}\right) / \operatorname{Br}(k)$ is surjective. We choose a finite subset $M \subset \operatorname{Br}_{1}(X, G) / \operatorname{Br}(k)$ such that $M$ surjects onto $\operatorname{Br}\left(X_{0}\right) / \operatorname{Br}(k)$.

Now let $x_{1} \in X(k)$ be any other point. Set $X_{1}:=x_{1} \cdot G^{\mathrm{ss}}$. It follows from Lemma 7.13 that $M \subset \operatorname{Br}_{1}(X, G) / \operatorname{Br}(k)$ surjects onto $\operatorname{Br}\left(X_{1}\right) / \operatorname{Br}(k)$.

7.17. Proof of Proposition 7.4. Let $x \in X(\mathbf{A})$ be orthogonal to $\operatorname{Br}_{1, x_{0}}(X, G)$. Let $u_{X}^{f}$ be an open neighbourhood of the $\mathbf{A}^{f}$-part $x^{f}$ of $x$. Let $U_{X}$ be the special neighbourhood of $x$ defined by $\mathcal{U}_{X}^{f}$.

Let $M \subset \operatorname{Br}_{1}(X, G) / \operatorname{Br}(k)$ be as in 7.16. Then $x$ is orthogonal to $M$. By Lemma 7.15 there exists an open neighbourhood $u^{f}$ of $x^{f}$ such that the corresponding special neighbourhood $U$ of $x$ in $X(\mathbf{A})$ is orthogonal to $M$. We may assume that $u_{X}^{f} \subset u^{f}$, then $u_{X} \subset \mathcal{U}$, hence $U_{X}$ is orthogonal to $M$.

Let $Y$ and $\psi: X \rightarrow Y$ be as in Construction 7.5 (i.e. $Y:=X / G^{\mathrm{ss}}$ ). Set $y:=$ $\psi(x) \in Y(\mathbf{A})$. Since $x$ is orthogonal to $\operatorname{Br}_{1, x_{0}}(X, G)$, we see by functoriality that $y$ is orthogonal to $\operatorname{Br}_{1, y_{0}}\left(Y, G^{\mathrm{sab}}\right)$. Clearly there is a semiabelian variety $G^{\prime}$ such that $Y$ is a (trivial) principal homogeneous space of $G^{\prime}$. We have a morphism of pairs $\left(Y, G^{\mathrm{sab}}\right) \rightarrow\left(Y, G^{\prime}\right)$, hence a homomorphism $\operatorname{Br}_{1}\left(Y, G^{\prime}\right) \rightarrow \operatorname{Br}_{1}\left(Y, G^{\mathrm{sab}}\right)$. But $\operatorname{Br}_{1}\left(Y, G^{\prime}\right)=\operatorname{Br}_{1}(Y)$, hence $y$ is orthogonal to the group $\operatorname{Br}_{1}(Y)$. As in the First reduction, see 7.1 , we define $U_{Y}^{f}:=\psi\left(\mathcal{U}_{X}^{f}\right)$ and we construct the corresponding special open neighbourhood $U_{Y}$ of $y$. By [7], Lemma A.2, for any $v \in \Omega_{\infty}$ we have $\psi\left(\mathcal{U}_{X, v}\right)=U_{Y, v}$. We see that $\psi\left(\mathcal{U}_{X}\right)$ is an open subset of $Y(\mathbf{A})$ of the form $u^{f} \times u_{\infty}$, where $u^{f} \subset Y\left(\mathbf{A}^{f}\right)$ is an open subset and $u_{\infty}=\prod_{v \in \Omega_{\infty}} u_{Y, v}$, where $\mathcal{U}_{Y, v} \subset Y\left(k_{v}\right)$ is the connected component of $y_{v}$. Then $\mathcal{U}_{\infty}$ is the connected component of $x_{\infty}$ in $X\left(k_{\infty}\right)$. Now since $Y$ is a torsor of the semiabelian variety $G^{\prime}$ with finite Tate-Shafarevich group, by [22], Theorem 4 , there exists a $k$-point $y_{1} \in Y(k) \cap \psi\left(u_{X}\right)$.

Let $X_{y_{1}}$ denote the fibre of $X$ over $y_{1}$. Consider the set $\mathcal{V}:=X_{y_{1}}(\mathbf{A}) \cap U_{X}$, it is open in $X_{y_{1}}(\mathbf{A})$. Since $y_{1} \in \psi\left(U_{X}\right)$, the set $\mathcal{V}$ is non-empty: there exists a point $x^{\prime}=\left(x_{v}^{\prime}\right) \in \mathcal{V}$. In particular, $X_{y_{1}}\left(k_{v}\right) \neq \varnothing$ for any $v \in \Omega_{r}$. The variety $X_{y_{1}}$ is a homogeneous space of $G^{\text {ss }}$ with geometric stabilizer $\bar{H} \cap \bar{G}^{\text {ss }}=\bar{H}^{\text {ssu }}$. The group $G^{\text {ss }}$ 
is semisimple simply connected by (iii). The group $\bar{H}^{\text {ssu }}$ is connected and characterfree, i.e. $\left(\bar{H}^{\text {ssu }}\right)^{\text {tor }}=1$. By [4], Corollary 7.4 , the fact that $X_{y_{1}}$ has points in all real completions of $k$ is enough to ensure that $X_{y_{1}}$ has a $k$-point. Note that $\operatorname{Br}\left(\bar{G}^{\text {ss }}\right)=0$ by [18], hence $\operatorname{Br}_{1}\left(X_{y_{1}}, G^{\text {ss }}\right)=\operatorname{Br}\left(X_{y_{1}}\right)$. Since $\mathcal{U}_{X}$ is orthogonal to $M$, we see that $\mathcal{V} \subset \mathcal{U}_{X}$ is orthogonal to $M$. Since $M$ surjects onto $\operatorname{Br}\left(X_{y_{1}}\right) / \operatorname{Br}(k)$, see 7.16, we see that $\mathcal{V}$ is orthogonal to $\operatorname{Br}\left(X_{y_{1}}\right)$. By Theorem 6.1 (due to Colliot-Thélène and $\mathrm{Xu})$ there is a point of the form $x_{1} . g_{S}$ in $\mathcal{V}$, where $x_{1} \in X_{y_{1}}(k)$ and $g_{S} \in G^{\mathrm{ss}}\left(k_{S}\right)$. It follows that the set $\mathcal{V} \cdot G^{\mathrm{ss}}\left(k_{S}\right)$ contains a $k$-point of $X_{y_{1}}$. Clearly $\mathcal{V} \cdot G^{\mathrm{ss}}\left(k_{S}\right) \subset$ $u_{X} \cdot G^{\mathrm{ss}}\left(k_{S}\right)$. Thus $U_{X} \cdot G^{\mathrm{ss}}\left(k_{S}\right)$ contains a $k$-point of $X$, which shows that the pair $(X, G)$ has Property $\left(\mathrm{P}^{S}\right)$. This completes the proof of Proposition 7.4.

Let us resume the proof of Theorem 7.3. We need a construction.

Construction 7.18. We follow an idea of a construction in the proof of [7], Theorem 3.5. By Lemma 3 in [9] there exists a coflasque resolution of the torus $H^{\text {tor }}$, i.e. an exact sequence of $k$-tori

$$
0 \rightarrow H^{\text {tor }} \rightarrow P \rightarrow Q \rightarrow 0
$$

where $P$ is a quasi-trivial torus and $Q$ is a coflasque torus. Recall that a torus is coflasque if for any field extension $K / k$ we have $H^{1}(K, \hat{Q})=0$, where $\hat{Q}$ is the character group of $Q$. Consider the $k$-group $F:=G \times P$. The group $H$ maps diagonally into $F$, and we can consider the quotient homogeneous space $W:=$ $H \backslash F$ of $F$. There is a natural morphism $t: W \rightarrow X$. We have $F^{\text {abvar }}=G^{\text {abvar }}$, hence $\amalg\left(F^{\text {abvar }}\right)$ is finite. We have a canonical homomorphism $H^{\text {tor }} \rightarrow F^{\text {sab }}$, and this homomorphism is clearly injective. Let us prove the following fact, which is necessary to apply Proposition 7.4 to the homogeneous space $W$ of $F$.

Lemma 7.19. With the notation of Construction 7.18, the pullback homomorphism

$$
\mathrm{Br}_{1, e}\left(F^{\mathrm{sab}}\right) \rightarrow \mathrm{Br}_{1, e}\left(H^{\mathrm{tor}}\right)
$$

is surjective.

Proof. By definition, we have the following exact commutative diagram:

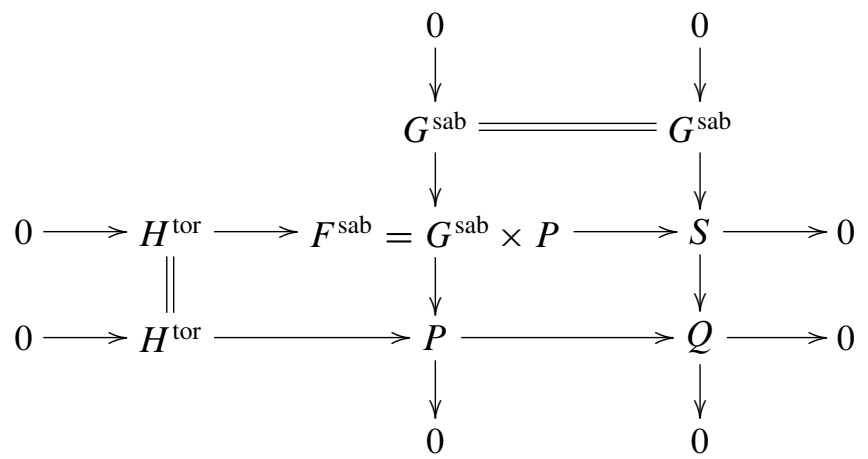


where $S$ is defined to be the quotient $F^{\mathrm{sab}} / H^{\text {tor }}$ and all the maps are the natural ones. The group $S$ is a semi-abelian variety. By assumption $Q$ is a coflasque torus. Therefore

$$
H^{3}(k, \hat{Q}) \cong \prod_{v \text { real }} H^{3}\left(k_{v}, \hat{Q}\right) \cong \prod_{v \text { real }} H^{1}\left(k_{v}, \hat{Q}\right)=0
$$

Denote by $M_{F}:=\left[0 \rightarrow F^{\text {sab }}\right]$ (resp. $M_{S}:=[0 \rightarrow S]$ ) the 1-motive (in degrees -1 and 0 ) associated to the semi-abelian variety $F^{\text {sab }}$ (resp. $S$ ), and by $M_{F}{ }^{*}$ (resp. $M_{S}{ }^{*}$ ) its Cartier dual (see [25], Section 1, page 97, for the definition of the Cartier dual of a 1-motive). We call a sequence of 1-motives over $k$ exact if the associated sequence of complexes of fppf sheaves on $\operatorname{Spec}(k)$ is exact.

Considering diagram (37) as an exact diagram in the category of 1-motives over $k$, we get a commutative exact diagram of 1-motives:

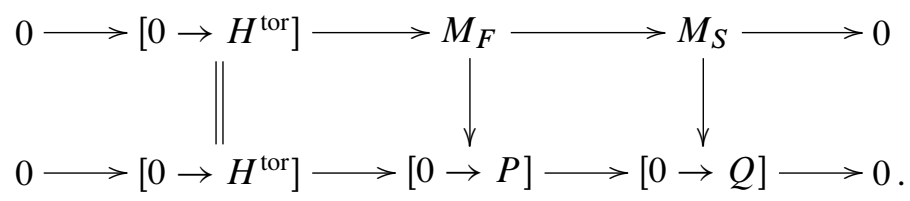

We can dualize this diagram to get the following commutative diagram of 1-motives:

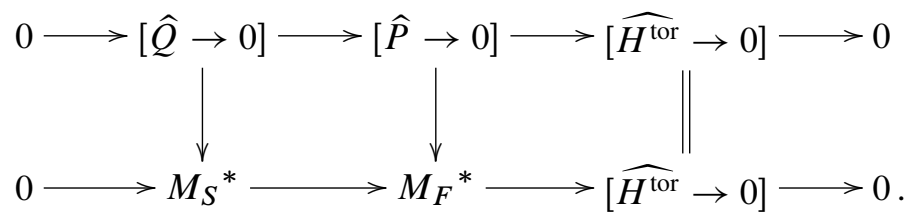

This diagram is exact as a diagram of complexes of fppf sheaves since the 1-motive $\left[0 \rightarrow H^{\text {tor }}\right]$ is associated to a $k$-torus (see [2], Remark 1.3.4). Hence this exact diagram induces a commutative exact diagram in hypercohomology:

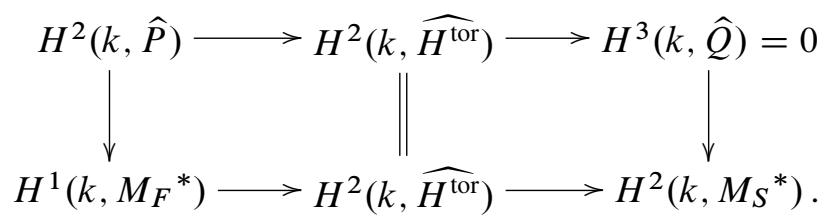

Therefore the map $H^{1}\left(k, M_{F}^{*}\right) \rightarrow H^{2}\left(k, \widehat{H^{\text {tor }}}\right)$ is surjective. But by [26], beginning of Section 4 , there are natural maps $\iota_{F}: H^{1}\left(k, M_{F}{ }^{*}\right) \rightarrow \operatorname{Br}_{1, e}\left(G^{\text {sab }}\right)$ and $\iota_{H^{\text {tor }}}: H^{2}\left(k, \widehat{H^{\text {tor }}}\right) \rightarrow \mathrm{Br}_{1, e}\left(H^{\text {tor }}\right)$ such that the second map is the canonical isomor- 
phism of [38], Lemma 6.9 (ii). Hence we get a commutative diagram

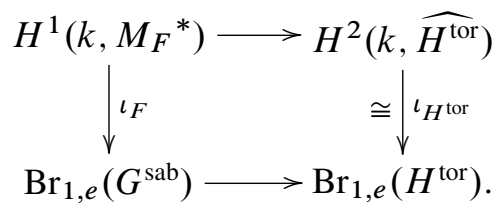

Since the top map is surjective, so is the bottom one.

Let $x \in X(\mathbf{A})$ be a point, and assume that $x$ is orthogonal to $\operatorname{Br}_{1}(X, G)$. The map $t: W \rightarrow X$ is a torsor under a quasi-trivial torus, and we want to lift $x$ to some $w \in W(\mathbf{A})$ orthogonal to $\operatorname{Br}_{1}(W, F)$. To do this, we need the following lemma.

Lemma 7.20. With the above notation, the torsor $t: W=H \backslash F \rightarrow X$ under the quasi-trivial torus $P$ induces a canonical exact sequence

$$
0 \rightarrow \mathrm{Br}_{1, x_{0}}(X, G) \stackrel{t^{*}}{\rightarrow} \mathrm{Br}_{1, w_{0}}(W, F) \stackrel{\varphi}{\rightarrow} \mathrm{Br}_{1, e}(P),
$$

where $x_{0}$ is the image of $e \in G(k)$ and $w_{0}$ is the image of $e \in F(k)$.

Proof. We first define the map $\varphi$ of the lemma. The pullback homomorphism: $\operatorname{Br}(W) \stackrel{\pi_{W}^{*}}{\longrightarrow} \operatorname{Br}(F)$ sends the subgroup $\operatorname{Br}_{1, w_{0}}(W, F)$ into $\operatorname{Br}_{1, e}(F)$. But $F=G \times P$, hence thanks to [38], Lemma 6.6, we have a natural isomorphism $\operatorname{Br}_{1, e}(F) \cong$ $\mathrm{Br}_{1, e}(G) \bigoplus \mathrm{Br}_{1, e}(P)$. We compose this map with the second projection

$$
\pi_{P}: \mathrm{Br}_{1, e}(G) \oplus \mathrm{Br}_{1, e}(P) \rightarrow \mathrm{Br}_{1, e}(P) .
$$

So we define a morphism $\varphi:=\operatorname{pr}_{P} \circ \pi_{W}^{*}: \mathrm{Br}_{1, w_{0}}(W, F) \rightarrow \mathrm{Br}_{1, e}(P)$. The morphism $t^{*}: \mathrm{Br}_{1, x_{0}}(X, G) \rightarrow \mathrm{Br}_{1, w_{0}}(W, F)$ in the lemma is induced by the morphism of pairs $t:(W, F) \rightarrow(X, G)$. By Theorem 2.8 we have an exact sequence

$$
\operatorname{Pic}(P) \rightarrow \operatorname{Br}(X) \stackrel{t^{*}}{\rightarrow} \operatorname{Br}(W) .
$$

The torus $P$ is quasi-trivial, therefore by Lemma 6.9 (ii) of [38], the group $\operatorname{Pic}(P)$ is trivial, so the homomorphism $t^{*}: \operatorname{Br}(X) \rightarrow \operatorname{Br}(W)$ is injective. In particular, the homomorphism $t^{*}: \mathrm{Br}_{1, x_{0}}(X, G) \rightarrow \mathrm{Br}_{1, w_{0}}(W, F)$ in Lemma 7.20 is injective. Therefore, it just remains to prove the exactness of the sequence of the lemma at the 
term $\mathrm{Br}_{1, w_{0}}(W, F)$. Consider the diagram

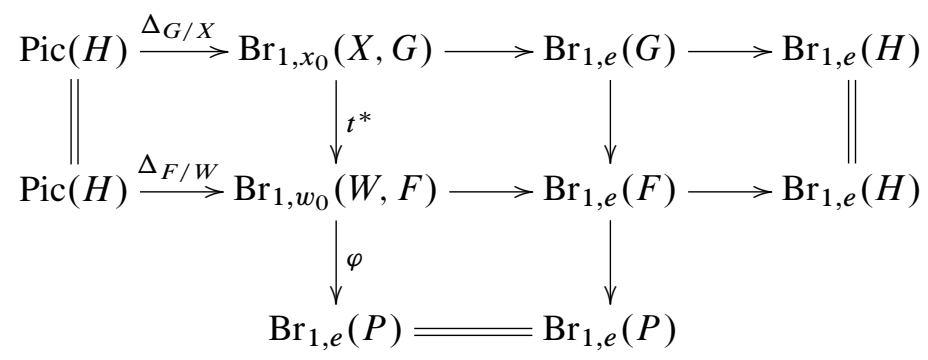

where the rows come from Theorem 2.8. The commutativity of this diagram is a consequence of the functoriality of the exact sequences of Theorem 2.8 and of the definition of the map $\varphi$. We conclude the proof of the exactness of the second column of the diagram by an easy diagram chase, using the exactness of the two first rows and that of the third column (see Corollary 2.12).

Corollary 7.21. With the above notation, if $x \in X(\mathbf{A})$ is orthogonal to $\operatorname{Br}_{1}(X, G)$, then there exists $w \in W(\mathbf{A})$ such that $t(w)=x$ and $w$ is orthogonal to $\operatorname{Br}_{1}(W, F)$.

Proof. Consider the exact sequence of Lemma 7.20. Taking dual groups, we obtain the dual exact sequence

$$
\mathrm{Br}_{1, e}(P)^{D} \stackrel{\varphi^{D}}{\longrightarrow} \mathrm{Br}_{1, w_{0}}(W, F)^{D} \stackrel{t_{*}}{\longrightarrow} \mathrm{Br}_{1, x_{0}}(X, G)^{D} \rightarrow 0,
$$

Let $m_{X, G}(x) \in \operatorname{Br}_{1, x_{0}}(X, G)^{D}$ denote the homomorphism $b \mapsto\langle b, x\rangle: \operatorname{Br}_{1, x_{0}}(X, G)$ $\rightarrow \mathbb{Q} / \mathbb{Z}$. By assumption $m_{G, X}(x)=0$. We wish to lift $x$ to some $w \in W(\mathbf{A})$ such that $m_{W, F}(w)=0$.

Since $H^{1}\left(k_{v}, P\right)=0$ for all $v$, we can lift $x$ to some point $w^{\prime} \in W(\mathbf{A})$ such that $t\left(w^{\prime}\right)=x$ (we use also Lang's theorem and Hensel's lemma). Then $t_{*}\left(m_{W, F}\left(w^{\prime}\right)\right)=$ $m_{X, G}(x) \in \operatorname{Br}_{1, x_{0}}(X, G)^{D}$. Since $m_{X, G}(x)=0$, we see from (38) that $m_{W, F}\left(w^{\prime}\right)=$ $\varphi^{D}(\xi)$ for some $\xi \in \operatorname{Br}_{1, e}(P)^{D} \cong \operatorname{Br}_{\mathrm{a}}(P)^{D}$. Let $p \in P(\mathbf{A})$. By Corollary 3.5 we have

$$
\left\langle b_{W}, w^{\prime} \cdot p\right\rangle=\left\langle b_{W}, w^{\prime}\right\rangle+\left\langle\varphi\left(b_{W}\right), p\right\rangle
$$

for any $b_{W} \in \mathrm{Br}_{1, w_{0}}(W, F)$. This means that

$$
m_{W, F}\left(w^{\prime} \cdot p\right)=m_{W, F}\left(w^{\prime}\right)+\varphi^{D}\left(m_{P}(p)\right)
$$

But we have seen that $m_{W, F}\left(w^{\prime}\right)=\varphi^{D}(\xi)$ for some $\xi \in \operatorname{Br}_{\mathrm{a}}(P)^{D}$. Now it follows from Lemma 4.1 that there exists $p \in P(\mathbf{A})$ such that $m_{P}(p)=-\xi$. Then

$$
m_{W, F}\left(w^{\prime} \cdot p\right)=\varphi^{D}(\xi)+\varphi^{D}(-\xi)=0 .
$$

We set $w:=w^{\prime} \cdot p \in W(\mathbf{A})$, then $m_{W, F}(w)=0$ and $t(w)=x$. 
7.22. We can now resume the proof of Theorem 7.3. We use Construction 7.18. Let $U_{X}^{f} \subset X\left(\mathbf{A}^{f}\right)$ be an open neighbourhood of $x^{f}$. Let $u_{X} \subset X(\mathbf{A})$ be the corresponding special neighbourhood of $x$. Set

$$
u_{W}^{f}:=t^{-1}\left(u_{X}^{f}\right) \subset W\left(\mathbf{A}^{f}\right)
$$

For $v \in \Omega_{\infty}$ let $U_{W, v}$ be the connected component of $w_{v}$ in $W\left(k_{v}\right)$, then by [7], Lemma A.2, we have $t\left(U_{W, v}\right)=U_{X, v}$. Set $U_{W, \infty}:=\prod_{v \in \Omega_{\infty}} U_{W, v}$. Set $U_{W}:=$ $u_{W}^{f} \times u_{W, \infty}$, then $u_{W}$ is the special open neighbourhood of $w$ defined by $u_{W}^{f}$, and $t\left(u_{W}\right) \subset u_{X}$.

The pair ( $W, F)$ of $F$ satisfies the hypotheses of Proposition 7.4 (see Lemma 7.19), so by that proposition, there is a point $w_{1} \in W(k) \cap \mathcal{U}_{W} \cdot F^{\mathrm{sc}}\left(k_{S}\right)$. Note that $F^{\mathrm{sc}}=G^{\mathrm{sc}}$. Set $x_{1}:=t\left(w_{1}\right)$, then $x_{1} \in X(k) \cap \mathcal{U}_{X} \cdot G^{\mathrm{sc}}\left(k_{S}\right)$. Thus the pair $(X, G)$ has Property $\left(\mathrm{P}^{S}\right)$.

This completes the proofs of Theorem 7.3 and proves the nontrivial inclusion of Theorem 1.4, that is, that any element of $\left(X(\mathbf{A})_{\bullet}\right)^{\mathrm{Br}_{1}(X, G)}$ lies in the closure of the set $X(k) . G^{\mathrm{scu}}\left(k_{S_{f}}\right)$. The argument in the proof of the trivial inclusion of Theorem 6.1 also proves the trivial inclusion of Theorem 1.4, that is, that each element of this closure is orthogonal to $\operatorname{Br}_{1}(X, G)$. This completes the proof of Main Theorem 1.4.

\section{The algebraic Manin obstruction}

In this section we prove Theorem 1.7 about the algebraic Manin obstruction ("algebraic" means coming from $\left.\mathrm{Br}_{1}(X)\right)$. We prove this result without using the result of Colliot-Thélène and $\mathrm{Xu}$ (Theorem 6.1 or [11], Theorem 3.7 (b)).

8.1. Before proving Theorem 1.7 , we need to prove a special case - an analogue of Theorem 6.1. In [6], the first-named author defined, for any connected group $H$ over a field $k$ of characteristic 0 , a Galois module $\pi_{1}(\bar{H})$, an abelian group $H_{\mathrm{ab}}^{1}(k, H)$ and a canonical abelianization map

$$
\mathrm{ab}^{1}: H^{1}(k, H) \rightarrow H_{\mathrm{ab}}^{1}(k, H)
$$

(see also [8] in any characteristic). These $\pi_{1}(\bar{H}), H_{\mathrm{ab}}^{1}(k, H)$ and $\mathrm{ab}^{1}$ are functorial in $H$.

Now let $k$ be a number field. Set $\Gamma:=\operatorname{Gal}(\bar{k} / k), \Gamma_{v}:=\operatorname{Gal}\left(\bar{k}_{v} / k_{v}\right)$. We regard $\Gamma_{v}$ as a subgroup of $\Gamma$.

For $v \in \Omega_{f}$ we defined in [6], Proposition 4.1 (i), a canonical isomorphism

$$
\lambda_{v}: H_{\mathrm{ab}}^{1}\left(k_{v}, H\right) \stackrel{\sim}{\longrightarrow}\left(\pi_{1}(\bar{H})_{\Gamma_{v}}\right)_{\mathrm{tors}},
$$


where $\pi_{1}(\bar{H})_{\Gamma_{v}}$ denotes the groups of coinvariants of $\Gamma_{v}$ in $\pi_{1}(\bar{H})$, and () tors denotes the torsion subgroup. Here we set $\lambda_{v}^{\prime}:=\lambda_{v}$.

For $v \in \Omega_{\infty}$ we defined in [6], Proposition 4.2, a canonical isomorphism

$$
\lambda_{v}: H_{\mathrm{ab}}^{1}\left(k_{v}, H\right) \stackrel{\sim}{\longrightarrow} H^{-1}\left(\Gamma_{v}, \pi_{1}(\bar{H})\right) .
$$

Here we define a homomorphism $\lambda_{v}^{\prime}$ as the composition

$$
\lambda_{v}^{\prime}: H_{\mathrm{ab}}^{1}\left(k_{v}, H\right) \stackrel{\lambda_{v}}{\longrightarrow} H^{-1}\left(\Gamma_{v}, \pi_{1}(\bar{H})\right) \hookrightarrow\left(\pi_{1}(\bar{H})_{\Gamma_{v}}\right)_{\text {tors }} .
$$

For any $v \in \Omega$ we define the Kottwitz map $\beta_{v}$ as the composition

$$
\beta_{v}: H^{1}\left(k_{v}, H\right) \stackrel{\mathrm{ab}^{1}}{\longrightarrow} H_{\mathrm{ab}}^{1}\left(k_{v}, H\right) \stackrel{\lambda_{v}^{\prime}}{\longrightarrow}\left(\pi_{1}(\bar{H})_{\Gamma_{v}}\right)_{\mathrm{tors}} .
$$

This map $\beta_{v}$ is functorial in $H$. Note that for $v \in \Omega_{f}$ the maps $\mathrm{ab}^{1}: H^{1}\left(k_{v}, H\right) \rightarrow$ $H_{\mathrm{ab}}^{1}\left(k_{v}, H\right)$ and $\beta_{v}$ are bijections. Thus for $v \in \Omega_{f}$ we have a canonical and functorial in $H$ bijection $\beta_{v}: H^{1}\left(k_{v}, H\right) \stackrel{\sim}{\longrightarrow}\left(\pi_{1}(\bar{H})_{\Gamma_{v}}\right)_{\text {tors }}$.

For any $v \in \Omega$ we define a map $\mu_{v}$ as the composition

$$
\mu_{v}: H^{1}\left(k_{v}, H\right) \stackrel{\beta_{v}}{\longrightarrow}\left(\pi_{1}(\bar{H})_{\Gamma_{v}}\right)_{\mathrm{tors}_{\mathrm{ta}}} \stackrel{\text { cor }_{v}}{\longrightarrow}\left(\pi_{1}(\bar{H})_{\Gamma}\right)_{\mathrm{tors}},
$$

where $\operatorname{cor}_{v}$ is the obvious corestriction map.

We write $\bigoplus_{v} H^{1}\left(k_{v}, H\right)$ for the set of families $\left(\xi_{v}\right)_{v \in \Omega}$ such that $\xi_{v}=1$ for almost all $v$. We define a map

$$
\mu:=\sum_{v \in \Omega} \mu_{v}: \bigoplus_{v \in \Omega} H^{1}\left(k_{v}, H\right) \rightarrow\left(\pi_{1}(\bar{H})_{\Gamma}\right)_{\mathrm{tors}} .
$$

Proposition 8.2 (Kottwitz [30], Proposition 2.6, see also [6], Theorem 5.15). The kernel of the map $\mu$ is equal to the image of the localization map

$$
H^{1}(k, H) \rightarrow \bigoplus_{v} H^{1}\left(k_{v}, H\right)
$$

Proposition 8.3. Let $G$ be a simply connected $k$-group over a number field $k$, and let $H \subset G$ be a connected geometrically character-free subgroup $\left(i . e . H^{\text {tor }}=1\right)$. Set $X:=H \backslash G$. Let $S$ be a finite set of places of $k$ containing at least one nonarchimedean place. Then any orbit of $G\left(\mathbf{A}^{S}\right)$ in $X\left(\mathbf{A}^{S}\right)$ contains a $k$-point.

Proof. Write $M:=\pi_{1}(\bar{H})$. First we prove that the map

$$
\mu_{S}=\sum_{v \in S} \mu_{v}: \prod_{v \in S} \operatorname{ker}\left[H^{1}\left(k_{v}, H\right) \rightarrow H^{1}\left(k_{v}, G\right)\right] \rightarrow\left(M_{\Gamma}\right)_{\text {tors }}
$$


is surjective. Since $H$ is geometrically character-free, the group $M=\pi_{1}(\bar{H})$ is finite, and therefore $\left(M_{\Gamma}\right)_{\text {tors }}=M_{\Gamma}$ and $\left(M_{\Gamma_{v}}\right)_{\text {tors }}=M_{\Gamma_{v}}$. In this case the map cor $_{v}$ is the canonical map $M_{\Gamma_{v}} \rightarrow M_{\Gamma}$, which is clearly surjective. Let $w \in S$ be a nonarchimedean place, then the map $\beta_{w}$ is bijective. It follows that the map $\mu_{w}: H^{1}\left(k_{w}, H\right) \rightarrow M_{\Gamma}$ is surjective (because $\left.\mu_{w}=\operatorname{cor}_{w} \circ \beta_{w}\right)$. Since $w$ is nonarchimedean, we have $H^{1}\left(k_{w}, G\right)=1$ (because $G$ is simply connected), hence $\operatorname{ker}\left[H^{1}\left(k_{w}, H\right) \rightarrow H^{1}\left(k_{w}, G\right)\right]=H^{1}\left(k_{w}, H\right)$. It follows that the map $\mu_{w}: \operatorname{ker}\left[H^{1}\left(k_{w}, H\right) \rightarrow H^{1}\left(k_{w}, G\right)\right] \rightarrow M_{\Gamma}$ is surjective. Now it is clear that the map $\mu_{S}=\sum_{v \in S} \mu_{v}$ is surjective.

We prove the proposition. We must prove that the localization map

$$
X(k) / G(k) \rightarrow X\left(\mathbf{A}^{S}\right) / G\left(\mathbf{A}^{S}\right)
$$

is surjective. In the language of Galois cohomology, we must prove that the localization map

$$
\operatorname{ker}\left[H^{1}(k, H) \rightarrow H^{1}(k, G)\right] \rightarrow \bigoplus_{v \notin S} \operatorname{ker}\left[H^{1}\left(k_{v}, H\right) \rightarrow H^{1}\left(k_{v}, G\right)\right]
$$

is surjective.

Let

$$
\xi^{S}=\left(\xi_{v}\right) \in \bigoplus_{v \notin S} \operatorname{ker}\left[H^{1}\left(k_{v}, H\right) \rightarrow H^{1}\left(k_{v}, G\right)\right] .
$$

Set $s:=\sum_{v \notin S} \mu_{v}\left(\xi_{v}\right) \in \pi_{1}(\bar{H})_{\Gamma}$. Since the map $\mu_{S}$ is surjective, there exists an element $\xi_{S}$ in the product $\prod_{v \in S} \operatorname{ker}\left[H^{1}\left(k_{v}, H\right) \rightarrow H^{1}\left(k_{v}, G\right)\right]$ such that $\sum_{v \in S} \mu_{v}\left(\xi_{v}\right)=-s$. Set

$$
\xi:=\left(\xi^{S}, \xi_{S}\right) \in \bigoplus_{v \in \Omega} \operatorname{ker}\left[H^{1}\left(k_{v}, H\right) \rightarrow H^{1}\left(k_{v}, G\right)\right] \subset \bigoplus_{v \in \Omega} H^{1}\left(k_{v}, H\right),
$$

then $\mu(\xi)=\sum_{v \in \Omega} \mu_{v}\left(\xi_{v}\right)=s+(-s)=0$. By Proposition 8.2 there exists a class $\xi_{0} \in H^{1}(k, H)$ with image $\xi$ in $\bigoplus_{v \in \Omega} H^{1}\left(k_{v}, H\right)$. Since the Hasse principle holds for $G$, we have $\xi_{0} \in \operatorname{ker}\left[H^{1}(k, H) \rightarrow H^{1}(k, G)\right]$. Since the image of $\xi_{0}$ in $\bigoplus_{v \in \Omega} H^{1}\left(k_{v}, H\right)$ is $\left(\xi^{S}, \xi_{S}\right)$, we see that the image of $\xi_{0}$ in $\bigoplus_{v \notin S} H^{1}\left(k_{v}, H\right)$ is $\xi^{S}$. Thus $\xi^{S}$ lies in the image of $\operatorname{ker}\left[H^{1}(k, H) \rightarrow H^{1}(k, G)\right]$.

Theorem 8.4. Let $G$ be a simply connected $k$-group over a number field $k$, and let $H \subset G$ be a connected geometrically character-free subgroup (i.e. $H^{\text {tor }}=1$ ). Set $X:=H \backslash G$. Let $S$ be a finite set of places of $k$ containing at least one nonarchimedean place $v_{0}$. Assume that $G^{\mathrm{ss}}(k)$ is dense in $G^{\mathrm{ss}}\left(\mathbf{A}^{S}\right)$. Then $X$ has strong approximation away from $S$ in the following sense. Let $x=\left(x_{v}\right) \in X(\mathbf{A})$ and let $U^{S} \subset X\left(\mathbf{A}^{S}\right)$ be any open neighbourhood of the $\mathbf{A}^{S}$-part $x^{S}$ of $x$. Then there exists a k-point $x_{0} \in X(k) \cap \mathcal{U}^{S}$. Moreover, one can ensure that for $v \in \Omega_{\infty} \cap S$ the 
points $x_{0}$ and $x_{v}$ lie in the same connected component of $X\left(k_{v}\right)$. More precisely, there exists $y_{v_{0}} \in X\left(k_{v_{0}}\right)$ such that the point $x^{\prime} \in X(\mathbf{A})$ defined by $x_{v_{0}}^{\prime}:=y_{v_{0}}$ and $x_{v}^{\prime}:=x_{v}$ for $v \neq v_{0}$ belongs to the closure of the set $X(k) . G\left(k_{S}\right)$ in $X(\mathbf{A})$ for the adelic topology.

Proof. Set $\Sigma:=\left\{v_{0}\right\}$. We denote by $x^{\Sigma} \in X\left(\mathbf{A}^{\Sigma}\right)$ and $x^{S} \in x\left(\mathbf{A}^{S}\right)$ the corresponding projections of $x$. By Proposition 8.3 applied to the finite set of places $\Sigma$, there exists a $k$-point $x_{0}^{\prime} \in X(k) \cap x^{\Sigma} \cdot G\left(\mathbf{A}^{\Sigma}\right)$. Let $y_{v_{0}}:=\left(x_{0}^{\prime}\right)_{v_{0}} \in X\left(k_{v_{0}}\right)$ and define $x^{\prime} \in X(\mathbf{A})$ as in the theorem. Then there exists $g \in G(\mathbf{A})$ such that $x_{0}^{\prime} . g=x^{\prime}$ in $X(\mathbf{A})$. Let $U \subset X(\mathbf{A})$ be an open neighbourhood of $x^{\prime}$. Since the orbit $x_{0}^{\prime} . G(\mathbf{A}) \subset X(\mathbf{A})$ is open (because $H$ is connected) and contains $x^{\prime}$, we may assume that $U \subset x_{0}^{\prime} . G(\mathbf{A})$.

By assumption $G(k) \cdot G\left(k_{S}\right)$ is dense in $G(\mathbf{A})$. It follows that there exists $g_{0} \in$ $G(k)$ and $g_{S} \in G\left(k_{S}\right)$ such that $x^{\prime \prime}:=x_{0}^{\prime} \cdot g_{0} \cdot g_{S}$ belongs to $\mathcal{U}$. Set $x_{0}:=x_{0}^{\prime} \cdot g_{0} \in$ $X(k)$, then $x^{\prime \prime}=x_{0} \cdot g_{S}$. We see that $x^{\prime \prime} \in X(k) . G\left(k_{S}\right) \cap \mathcal{U}$. Therefore, we conclude that $x^{\prime}$ lies in the closure of $X(k) . G\left(k_{S}\right)$.

Concerning the infinite places, for $v \in \Omega_{\infty} \cap S$ we have $x_{0} \in x_{v} . G\left(k_{v}\right)$, because $x_{0}^{\prime} \in x_{v} \cdot G\left(k_{v}\right)$. Since $G$ is simply connected, the group $G\left(k_{v}\right)$ is connected (see [34], Theorem 5.2.3), hence the image of $x_{0}$ in $X\left(k_{v}\right)$ is contained in the connected component of $x_{v}$ in $X\left(k_{v}\right)$.

8.5. Proof of Theorem 1.7. To prove this theorem, we can follow the proof of Theorem 1.4 to make reductions, so that we may assume the following:

(i) $G^{\mathrm{u}}=\{1\}$,

(ii) $H \subset G^{\text {lin }}$, i.e. $H$ is linear,

(iii) $G^{\text {ss }}$ is simply connected,

(iv) $\amalg\left(G^{\text {abvar }}\right)$ is finite,

(v) the homomorphism $H^{\text {tor }} \rightarrow G^{\text {sab }}$ is injective.

Set $\Sigma^{\prime}:=\Omega_{\infty} \cup\left\{v_{0}\right\}$. Let $U_{X}^{\Sigma^{\prime}} \subset X\left(\mathbf{A}^{\Sigma^{\prime}}\right)$ be an open neighbourhood of the projection $x^{\Sigma^{\prime}} \in X\left(\mathbf{A}^{\Sigma^{\prime}}\right)$ of $x$. Set $\mathcal{U}_{X}^{f}:=\mathcal{U}_{X}^{\Sigma^{\prime}} \times X\left(k_{v_{0}}\right)$. Let $u_{X}$ be the special open neighbourhood of $x$ in $X(\mathbf{A})$ defined by $\mathcal{U}_{X}^{f}$. Set $Y:=G^{\mathrm{sab}} / H^{\text {tor }}$, and consider the canonical morphism $\psi: X \rightarrow Y$. Set $y:=\psi(x) \in Y(\mathbf{A})$, then $y$ is orthogonal to the group $\operatorname{Br}_{1}(Y)$ for the Manin pairing. Hence by [22], Theorem 4, there exists $y_{0} \in Y(k) \cap \psi\left(\mathcal{U}_{X}\right)$. Set $X_{y_{0}}:=\psi^{-1}\left(y_{0}\right) \subset X$ and $\mathcal{V}:=X_{y_{0}}(\mathbf{A}) \cap \mathcal{U}_{X}$. Then $\mathcal{V}$ is open and non-empty since $y_{0} \in \psi\left(\mathcal{U}_{X}\right)$. As in the proof of Proposition 7.4, we know that $X_{y_{0}}$ is a homogeneous space of the semisimple simply connected group $G^{\mathrm{ss}}=G^{\mathrm{sc}}$, with connected character-free geometric stabilizers, and with a $k$ point. Therefore Theorem 8.4 implies that $X_{y_{0}}(k) \cdot G^{\mathrm{sc}}\left(k_{S}\right) \cap \mathcal{V} \neq \emptyset$. In particular, the set $X(k) \cdot G^{\mathrm{sc}}\left(k_{S}\right) \cap U_{X}$ is non-empty. Set $S^{\prime}:=S \backslash\left\{v_{0}\right\}, S_{f}^{\prime}:=S^{\prime} \cap \Omega_{f}$, $u_{X}^{\left\{v_{0}\right\}}:=U_{X}^{\Sigma^{\prime}} \times \mathcal{U}_{X, \infty}$, then it follows that the set $X(k) . G^{\mathrm{sc}}\left(k_{S^{\prime}}\right) \cap \mathcal{U}_{X}^{\left\{v_{0}\right\}} \subset X\left(\mathbf{A}^{\left\{v_{0}\right\}}\right)$ 
is non-empty. Since $U_{X}^{\left\{v_{0}\right\}} \cdot G^{\mathrm{sc}}\left(k_{S^{\prime}}\right)=U_{X}^{\left\{v_{0}\right\}} \cdot G^{\mathrm{sc}}\left(k_{S_{f}^{\prime}}\right)$, we obtain easily that the set $X(k) \cdot G^{\text {sc }}\left(k_{S_{f}^{\prime}}\right) \cap U_{X}^{\left\{v_{0}\right\}} \subset X\left(\mathbf{A}^{\left\{v_{0}\right\}}\right)$ is non-empty. This completes the proof of Theorem 1.7 .

Acknowledgements. The first-named author is grateful to the Tata Institute of Fundamental Research, Mumbai, where a part of this paper was written, for hospitality and good working conditions. The second-named author thanks David Harari for many helpful suggestions. Both authors thank the referee for his/her comments and suggestions.

\section{References}

[1] M. Artin, A. Grothendieck et J.-L. Verdier (éds.), Théorie des topos et cohomologie étale des schémas. Séminaire de géométrie algébrique du Bois-Marie (SGA 4), Tome 1, 2, 3, Lecture Notes in Math. 269, 270, 305, Springer-Verlag, Berlin 1972-73. Zbl 0234.00007 Zbl 0237.00012 Zbl 0245.00002 MR 0354654 MR 0354653 MR 0354652

[2] L. Barbieri-Viale and A. Bertapelle, Sharp de Rham realization. Adv. Math. 222 (2009), 1308-1338. Zbl 1216.14006 MR 2554937

[3] E. Bierstone and P. D. Milman, Canonical desingularization in characteristic zero by blowing up the maximum strata of a local invariant. Invent. Math. 128 (1997), 207-302. Zbl 0896.14006 MR 1440306

[4] M. Borovoi, Abelianization of the second nonabelian Galois cohomology. Duke Math. J. 72 (1993), 217-239. Zbl 0849.12011 MR 1242885

[5] M. Borovoi, The Brauer-Manin obstruction for homogeneous spaces with connected or abelian stabilizer. J. Reine Angew. Math. 473 (1996), 181-194. Zbl 0844.14020 MR 1390687

[6] M. Borovoi, Abelian Galois cohomology of reductive groups. Mem. Amer. Math. Soc. 132 (1998), no. 626. Zbl 0918.20037 MR 1401491

[7] M. Borovoi, J.-L. Colliot-Thélène and A. N. Skorobogatov, The elementary obstruction and homogeneous spaces. Duke Math. J. 141 (2008), 321-364. Zbl 1135.14013 MR 2376817

[8] J.-L. Colliot-Thélène, Résolutions flasques des groupes linéaires connexes. J. Reine Angew. Math. 618 (2008), 77-133. Zbl 1158.14021 MR 2404747

[9] J.-L. Colliot-Thélène et J.-J. Sansuc, La R-équivalence sur les tores. Ann. Sci. École Norm. Sup. (4) 10 (1977), 175-229. Zbl 0356.14007 MR 0450280

[10] J.-L. Colliot-Thélène et J.-J. Sansuc, La descente sur les variétés rationnelles, II. Duke Math. J. 54 (1987), 375-492. Zbl 0659.14028 MR 0899402

[11] J.-L. Colliot-Thélène and F. Xu, Brauer-Manin obstruction for integral points of homogeneous spaces and representation by integral quadratic forms. Compositio Math. 145 (2009), 309-363. Zbl 1190.11036 MR 2501421

[12] A. J. de Jong, A result of Gabber. Preprint. www.math.columbia.edu/ dejong/papers/2-gabber.pdf. 
[13] P. Deligne, Cohomologie étale. Séminaire de Géometrie Algébrique du Bois-Marie

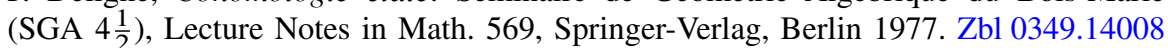
MR 0463174

[14] C. Demarche, Suites de Poitou-Tate pour les complexes de tores à deux termes. Internat. Math. Res. Notices 2011 (2011), no. 1, 135-174. Zbl 1213.14079 MR 2755486

[15] J. Dieudonné and A. Grothendieck, Éléments de géométrie algébrique (EGA). Inst. Hautes Études Sci. Publ. Math. 4, 8, 11, 17, 20, 24, 28, 32 (1960-1967).

Zbl 0118.36206 Zbl 0136.15901 Zbl 0135.39701 Zbl 0144.19904 Zbl 0153.22301 MR 0217083 MR 0217084 MR 0217085 MR 0163911 MR 0173675 MR 0217086 MR 0238860

[16] A. Ducros, Principe local-global sur le corps des fonctions d'une courbe réelle: espaces principaux homogènes et surfaces rationnelles. Thèse de l'Université Paris-Sud, 1997.

[17] S. Encinas and O. Villamayor, Good points and constructive resolution of singularities. Acta Math. 181 (1998), 109-158. Zbl 0930.14038 MR 1654779

[18] S. Gille, On the Brauer group of a semisimple algebraic group. Adv. Math. 220 (2009), 913-925. Zbl 1173.11023 MR 2483231

[19] J. Giraud, Cohomologie non abélienne. Grundlehren Math. Wiss. 179, Springer-Verlag, Berlin 1971. Zbl 0226.14011 MR 0344253

[20] A. Grothendieck, Le groupe de Brauer. I, II, III. In Dix exposés sur la cohomologie des schémas, North-Holland, Amsterdam; Masson, Paris 1968, 46-188. Zbl 0193.21503 Zbl 0198.25803 Zbl 0198.25901 MR 0244269 MR 0244270 MR 0244271

[21] D. Harari, The Manin obstruction for torsors under connected algebraic groups. Internat. Math. Res. Notices 2006 (2006), Article ID 68632, 1-13. Zbl 1100.14013 MR 2211158

[22] D. Harari, Le défaut d'approximation forte pour les groupes algébriques commutatifs. Algebra Number Theory 2 (2008), 595-611. Zbl 1194.14067 MR 2429455

[23] D. Harari and A. N. Skorobogatov, The Brauer group of torsors and its arithmetic applications. Ann. Inst. Fourier 53 (2003), 1987-2019. Zbl 1115.11032 MR 2044165

[24] D. Harari and A. N. Skorobogatov, Non-abelian descent and the arithmetic of Enriques surfaces. Internat. Math. Res. Notices 52 (2006), 3203-3228. Zbl 1099.14008 MR 2186792

[25] D. Harari and T. Szamuely, Arithmetic duality theorems for 1-motives. J. Reine Angew. Math. 578 (2005), 93-128. Zbl 1088.14012 MR 2113891

[26] D. Harari and T. Szamuely, Local-global principles for 1-motives. Duke Math. J. 143 (2008), 531-557. Zbl 1155.14020 MR 2423762

[27] H. Hironaka, Resolution of singularities of an algebraic variety over a field of characteristic zero. I, II. Ann. of Math. (2) 79 (1964), 109-203; ibid. (2) 79 (1964) 205-326. Zbl 0122.38603 MR 0199184

[28] R. T. Hoobler, A cohomological interpretation of Brauer groups of rings. Pacific J. Math. 86 (1980), 89-92. Zbl 0459.13002 MR 0586870

[29] B. Kahn, Deux théorèmes de comparaison en cohomologie étale; applications. Duke Math. J. 69 (1993), 137-165. Zbl 0789.14014 MR 1201695

[30] R. E. Kottwitz, Stable trace formula: elliptic singular terms. Math. Ann. 275 (1986), 365-399. Zbl 0577.10028 MR 0858284 
[31] S. Lang, Algebraic groups over finite fields. Amer. J. Math 78 (1956), 555-563. Zbl 0073.37901 MR 0086367

[32] M. Nagata, A generalization of the imbedding problem of an abstract variety in a complete variety. J. Math. Kyoto Univ. 3 (1963), 89-102. Zbl 0223.14011 MR 0158892

[33] O. T. O'Meara, Introduction to quadratic forms. Grundlehren Math. Wiss. 117, SpringerVerlag, Berlin 1963. Zbl 0107.03301 MR 0152507

[34] A. L. Onishchik and È. B. Vinberg, Lie groups and algebraic groups. Springer Ser. Soviet Math., Springer-Verlag, Berlin 1990. Zbl 0722.22004 MR 1064110

[35] F. Oort, Commutative group schemes. Lecture Notes in Math. 15, Springer-Verlag, Berlin 1966. Zbl 0216.05603 MR 0213365

[36] V. Platonov and A. Rapinchuk, Algebraic groups and number theory. Academic Press, Boston 1994. Zbl 0841.20046 MR 1278263

[37] M. Rosenlicht, Toroidal algebraic groups. Proc. Amer. Math. Soc. 12 (1961), 984-988. Zbl 0107.14703 MR 0133328

[38] J.-J. Sansuc, Groupe de Brauer et arithmétique des groupes algébriques linéaires sur un corps de nombres. J. Reine Angew. Math. 327 (1981), 12-80. Zbl 0468.14007 MR 0631309

[39] I. R. Shafarevich, Basic algebraic geometry. 2. Schemes and complex manifolds. 2nd ed., Springer-Verlag, Berlin 1994 Zbl 0797.14002 MR 1328834

[40] A. N. Skorobogatov, Torsors and rational points. Cambridge Tracts in Math. 144, Cambridge University Press, Cambridge 2001. Zbl 0972.14015 MR 1845760

Received December 2, 2009

Mikhail Borovoi, Raymond and Beverly Sackler School of Mathematical Sciences, Tel Aviv University, 69978 Tel Aviv, Israel

E-mail: borovoi@post.tau.ac.il

Cyril Demarche, Université Paris-Sud, Laboratoire de Mathématiques d'Orsay, 91405 Orsay

Cedex, France

E-mail: demarche@math.jussieu.fr 